\title{
Mitochondrial Superoxide Production Decreases on Glucose-Stimulated Insulin Secretion in Pancreatic $\beta$ Cells Due to Decreasing Mitochondrial Matrix NADH/NAD ${ }^{+}$Ratio
}

Lydie Plecitá-Hlavatá, ${ }^{1, *}$ Hana Engstová, ${ }^{1, *}$ Blanka Holendová, ${ }^{1, *}$ Jan Tauber, ${ }^{1}$ Tomáš Špaček, Lucie Petrásková, ${ }^{2}$ Vladimír Křen, ${ }^{2}$ Jitka Špačková, ${ }^{1}$ Klára Gotvaldová, Jan Ježek, ${ }^{1,3}$ Andrea Dlasková, Katarína Smolková, ${ }^{1}$ and Petr Ježek ${ }^{1}$

\begin{abstract}
Aims: Glucose-stimulated insulin secretion (GSIS) in pancreatic $\beta$ cells was expected to enhance mitochondrial superoxide formation. Hence, we elucidated relevant redox equilibria.

Results: Unexpectedly, INS-1E cells at transitions from 3 (11 $\mathrm{m} M$; pancreatic islets from $5 \mathrm{~m} M)$ to $25 \mathrm{~m} M$ glucose decreased matrix superoxide release rates (MitoSOX Red monitoring validated by MitoB) and $\mathrm{H}_{2} \mathrm{O}_{2}$ (mitoHyPer, subtracting mitoSypHer emission). Novel double-channel fluorescence lifetime imaging, approximating free mitochondrial matrix $\mathrm{NADH}_{\mathrm{F}}$, indicated its $\sim 20 \%$ decrease. Matrix $\mathrm{NAD}^{+}{ }_{\mathrm{F}}$ increased on GSIS, indicated by the FAD-emission lifetime decrease, reflecting higher quenching of FAD by $\mathrm{NAD}^{+}$. The participation of pyruvate/malate and pyruvate/citrate redox shuttles, elevating cytosolic $\mathrm{NADPH}_{\mathrm{F}}$ (iNAP1 fluorescence monitoring) at the expense of matrix $\mathrm{NADH}_{\mathrm{F}}$, was indicated, using citrate (2-oxoglutarate) carrier inhibitors and cytosolic malic enzyme silencing: All changes vanished on these manipulations. ${ }^{13} \mathrm{C}$ incorporation from ${ }^{13} \mathrm{C}$-L-glutamine into ${ }^{13} \mathrm{C}$-citrate reflected the pyruvate/isocitrate shuttle. Matrix $\mathrm{NADPH}_{\mathrm{F}}$ (iNAP3 monitored) decreased. With decreasing glucose, the suppressor of Complex III site Q electron leak (S3QEL) suppressor caused a higher Complex I I $\mathrm{I}_{\mathrm{F}}$ site contribution, but a lower superoxide fraction ascribed to the Complex III site $\mathrm{III}_{\mathrm{Qo}}$. Thus, the diminished matrix $\mathrm{NADH}_{\mathrm{F}} / \mathrm{NAD}^{+}{ }_{\mathrm{F}}$ decreased Complex I flavin site $\mathrm{I}_{\mathrm{F}}$ superoxide formation on GSIS.

Innovation: Mutually validated methods showed decreasing superoxide release into the mitochondrial matrix in pancreatic $\beta$ cells on GSIS, due to the decreasing matrix $\mathrm{NADH}_{\mathrm{F}} / \mathrm{NAD}^{+}{ }_{\mathrm{F}}\left(\mathrm{NADPH}_{\mathrm{F}} / \mathrm{NADP}^{+}\right)$at increasing cytosolic $\mathrm{NADPH}_{\mathrm{F}}$ levels. The developed innovative methods enable real-time $\mathrm{NADH} / \mathrm{NAD}^{+}$and NADPH/ $\mathrm{NADP}^{+}$monitoring in any distinct cell compartment.

Conclusion: The export of reducing equivalents from mitochondria adjusts lower mitochondrial superoxide production on GSIS, but it does not prevent oxidative stress in pancreatic $\beta$ cells. Antioxid. Redox Signal. 33, 789-815.
\end{abstract}

Keywords: mitochondrial superoxide generation, pancreatic $\beta$ cells, glucose-stimulated insulin secretion, Complex I, NADH/NAD ${ }^{+}$ratio, fluorescence lifetime imaging

\footnotetext{
${ }^{1}$ Department of Mitochondrial Physiology, No. 75, Institute of Physiology of the Czech Academy of Sciences, Prague, Czech Republic.

${ }^{2}$ Laboratory of Biotransformation, Institute of Microbiology of the Czech Academy of Sciences, Prague, Czech Republic.

${ }^{3}$ The Gurdon Institute, University of Cambridge, Cambridge, United Kingdom.

*These authors contributed equally.
}

(C) Lydie Plecitá-Hlavatá et al. 2020; Published by Mary Ann Liebert, Inc. This Open Access article is distributed under the terms of the Creative Commons License (http://creativecommons.org/licenses/by/4.0), which permits unrestricted use, distribution, and reproduction in any medium, provided the original work is properly cited. 


\section{Innovation}

The concomitant decline in superoxide $/ \mathrm{H}_{2} \mathrm{O}_{2}$ release to the mitochondrial matrix was documented by three mutually validated methods to be dependent on the operation of three redox shuttles on glucose-stimulated insulin secretion in INS-1E cells. Fluorescent iNAP3 and iNAP1 probes indicated a decrease in matrix and an increase in cytosolic $\mathrm{NADPH}_{\mathrm{F}}$. The latter was confirmed by novel double-channel fluorescence lifetime imaging, which together with elevated $\beta$-hydroxybutyrate indicated increasing matrix $\mathrm{NAD}_{\mathrm{F}}^{+}$at lowered $\mathrm{NADH}_{\mathrm{F}}$, hence decreasing $\mathrm{NADH}_{\mathrm{F}} / \mathrm{NAD}_{\mathrm{F}}^{+}$ratios. Inspections of superoxide formation sites by S3(1)QEL identified the

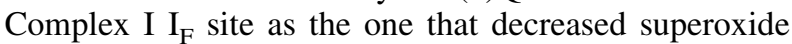
formation when there was a diminished $\mathrm{NADH}_{\mathrm{F}} / \mathrm{NAD}_{\mathrm{F}}^{+}$ ratio.

\section{Introduction}

$\mathbf{T}$ He Bioenergetics of PANCREATIC $\beta$ CELls was considered to be relatively well understood $(10,32,33)$. Mitochondria of $\beta$ cells represent the perfect glucose sensor, participating in glucose-stimulated insulin secretion (GSIS) $(2,20,28,32,33,52,55)$. Glucose sensing was considered to be exclusively coupled with insulin exocytosis by elevated ATP, stemming from augmented glucose metabolism and oxidative phosphorylation (OXPHOS). However, recently we revealed that, in addition to ATP, also a parallel redox signaling from NADPH oxidase 4 (NOX4) is essential for GSIS (48). In $\beta$ cells, the insulin-independent glucose transporter GLUT2 (in rodents) allows cell glucose equilibration with plasma glucose levels. Glycolysis is $100 \%$ restricted to the pyruvate dehydrogenase (PDH) and pyruvate carboxylase reaction followed by OXPHOS (Fig. 1A, B) (2, $10,32,33,52,55)$. The concomitantly increased ATP/ADP ratio within the sub-plasma-membrane cytosolic microdomain was believed to be sufficient to induce the closure of ATP-sensitive potassium $\left(\mathrm{K}_{\mathrm{ATP}}\right)$ channels, thus depolarizing the plasma membrane and activating voltage-gated L-type $\mathrm{Ca}^{2+}$ channels $\left(\mathrm{Ca}_{\mathrm{L}}\right)(2,52,55)$. However, we demonstrated that a parallel $\mathrm{H}_{2} \mathrm{O}_{2}$ burst is essentially required to close the $\mathrm{K}_{\mathrm{ATP}}$ channel, together with ATP. Any single component (sole ATP or sole $\mathrm{H}_{2} \mathrm{O}_{2}$ ) is not sufficient to stimulate insulin secretion (48). The resulting $\mathrm{Ca}^{2+}$ influx elevates cytosolic $\mathrm{Ca}^{2+}$ concentration and stimulates $\mathrm{Ca}^{2+}$-dependent exocytosis of insulin-containing granules.

$\beta$ Cell mitochondria represent a highly relevant source of reactive oxygen species (ROS) $(1,16,30,31,39)$. Superoxide is primarily formed and converted to $\mathrm{H}_{2} \mathrm{O}_{2}$ by manganese superoxide dismutase (MnSOD) or copper-zinc superoxide dismutase (CuZnSOD) in the mitochondrial matrix or intermembrane space, respectively $(8,11,30,31)$. The resulting $\mathrm{H}_{2} \mathrm{O}_{2}$ may also serve as a signaling molecule (50). Indeed, insulin release is also stimulated due to the elevated superoxide formation by $\beta$-like oxidation of branched-chain ketoacids in mitochondria, wherein superoxide is converted to "signaling" $\mathrm{H}_{2} \mathrm{O}_{2}$, diffusing up to the $\mathrm{K}_{\text {ATP }}$ channel to aid its closure (48). However, whether such matrix redox burst exists in glucose metabolism is not yet resolved.

Concomitantly, three described redox shuttles export reducing equivalents from the mitochondrial matrix into the cytosol on GSIS in pancreatic $\beta$ cells (34): (i) the pyruvate/ malate, (ii) pyruvate/citrate, and (iii) pyruvate/isocitrate shuttle (Fig. 1A, B) (35). Their operation should elevate cytosolic NADPH production at the expense of matrix NADH. The first two shuttles deliver substrates for the cytosolic malic enzyme (ME1) (34), and the third one delivers substrates for isocitrate dehydrogenase 1 (IDH1) (22). ME1 and IDH1 subsequently produce NADPH. The pyruvate carrier (PyrC) and 2-oxoglutarate carrier (2OGC) and matrix malate dehydrogenase $(\mathrm{MDH})$ are essential for the pyruvate/malate shuttle (34), PyrC and citrate carrier (CitC) and ATP-citrate lyase represent the pyruvate/citrate shuttle (34) and 2OGC and CitC plus matrix NADPH-consuming isocitrate dehydrogenase 2 (IDH2), acting in the reductive carboxylation mode (59), are essential for the pyruvate/isocitrate shuttle (Fig. 1A) (22, 34). Invariably, these redox shuttles presumably elevate cytosolic NADPH when active. GSIS is then slightly facilitated by the resulting NADPH increase $(22,35,52)$

FIG. 1. Schematic illustrating the role of mitochondrial redox shuttles in pancreatic $\beta$ cells on GSIS. Mitochondrial redox shuttles are depicted by arrows of distinct colors: dark green-pyruvate/malate shuttle; purple-pyruvate/citrate shuttle; and orange-pyruvate/isocitrate shuttle. The malate-asparate shuttle is brown. (A) High glucose state, that is, stimulating insulin release. Mitochondrial pyruvate redox shuttles reportedly export reducing equivalents from the mitochondrial matrix into the cytosol on GSIS (34). Consequently, cytosolic NADPH should be hypothetically elevated at the expense of mitochondrial matrix NADH. The resulting elevated cytosolic NADPH has been reported to facilitate GSIS (52). Oxidative glucose metabolism leads to cytosolic ATP plus $\mathrm{H}_{2} \mathrm{O}_{2}$ elevation (48), thus initiating $\mathrm{K}_{\text {ATP }}$ channel-dependent GSIS $(2,52,55)$. Glutamate dehydrogenase is depicted as producing glutamate (44); NNT is depicted to allow both reverse and forward mode (58). (B) Low glucose state: Instead of the redox shuttles cited earlier, the malate-aspartate shuttle (brown) may play a dominant role in mediating metabolic fluxes at low, nonstimulating, glucose concentration, which results in the transfer of redox equivalents of NADH into the mitochondrial matrix. Note that both the malate-aspartate carrier and 2OGC conduct the opposite fluxes within the malate-aspartate shuttle compared with the three pyruvate redox shuttles acting on GSIS. "Continuation of flux within the malate-aspartate shuttle. *Indicates IDH2 reaction. 2OG, 2oxoglutarate; 2-OG C, 2-oxoglutarate carrier; 2OGDH, 2-oxoglutarate dehydrogenase; AcCoA, acetyl-CoA; ACL, ATPcitrate lyase; ASCT, alanine serine cysteine transporter; CS, citrate synthase; CitC, citrate carrier; FA, fatty acid; FASN, fatty acid synthase; FH, fumarate hydratase; GDH, glutamate dehydrogenase; Gln C, glutamine carrier; GLUT, glucose transporter; GSIS, glucose-stimulated insulin secretion; $\mathrm{K}_{\mathrm{ATP}}$, ATP-sensitive potassium; IDH2, isocitrate dehydrogenase 2; MAC, malate aspartate carrier; MDH, malate dehydrogenase; ME, malic enzyme; NNT, nicotinamide nucleotide transhydrogenase; PC, pyruvate carboxylase; PDH, pyruvate dehydrogenase; PyrC, pyruvate carrier; S-CoA, succinyl coenzyme A; SDH, succinate dehydrogenase; SN2, system N transporter 2; SUCC, succinate. Color images are available online. 
A

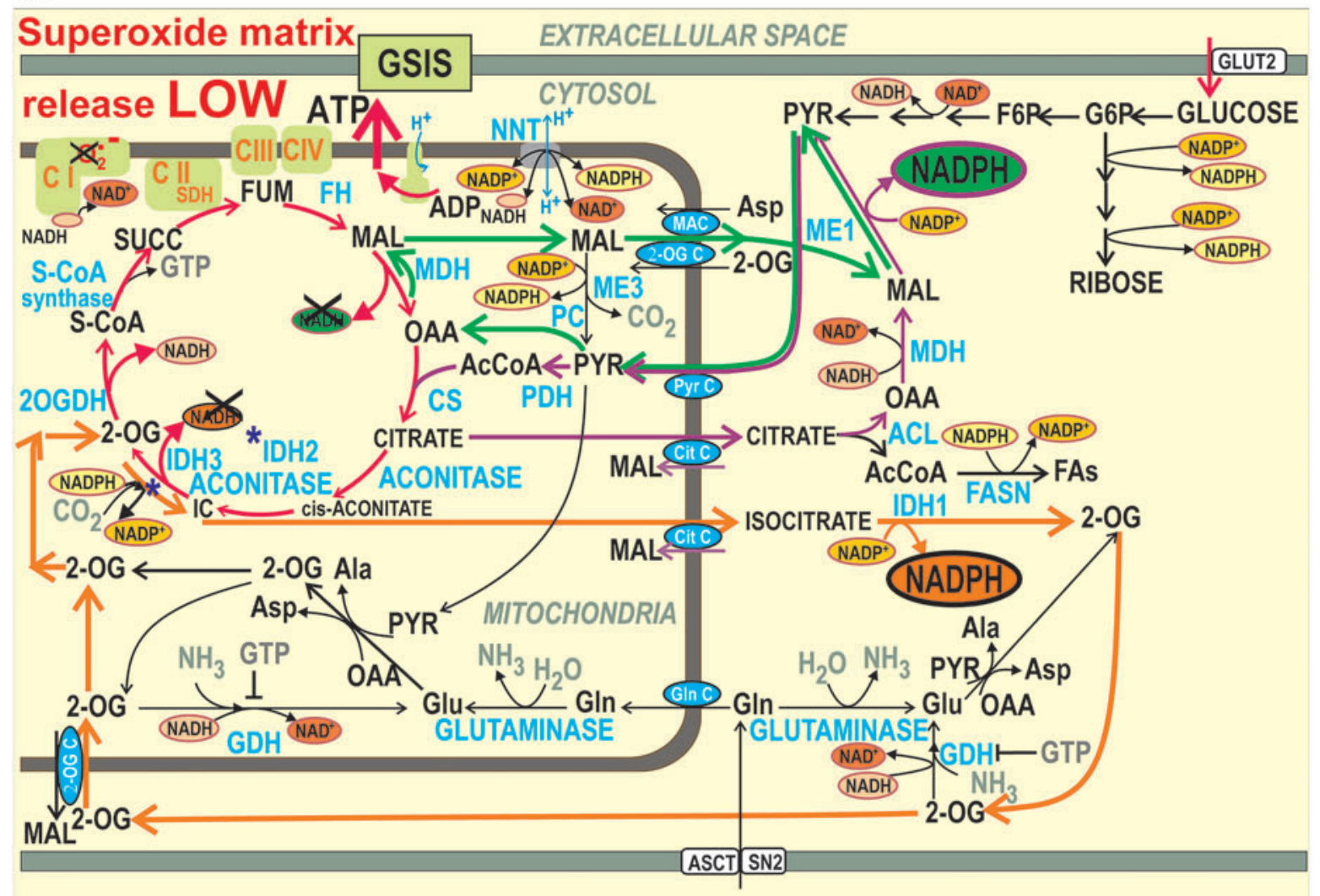

B

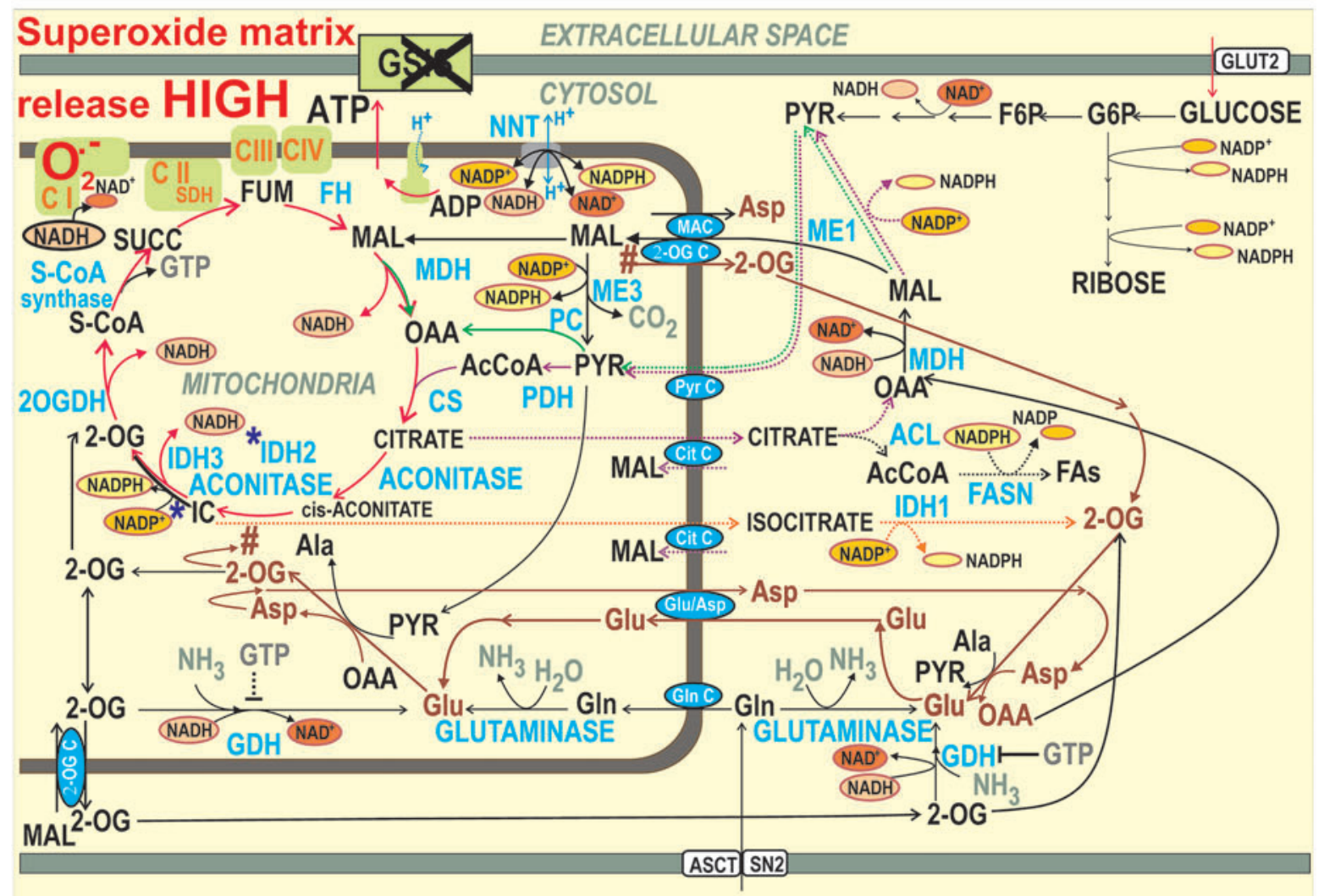


that adds to the production of pentose-phosphate pathway (PPP) supplying NOX4 (48). For each molecule of malate or citrate/isocitrate exported from the mitochondrial matrix by the respective shuttle, one molecule of NADH should be missing for Complex I, since MDH or isocitrate dehydrogenase 3 (IDH3) (otherwise producing NADH) cannot use their substrates. Thus, mitochondrial matrix $\mathrm{NADH}$ formation is predicted to be lower and the matrix $\mathrm{NAD}^{+}$is assumed to accumulate on GSIS. In this work, we aimed at experimentally supporting this scheme (Fig. 1A; Hypothesis I).

Mitochondrial superoxide formation takes place as an inevitable side reaction of electrons with oxygen within the sites of mitochondrial respiratory chain Complexes I and III, by dysfunctional Complex II, and sites of matrix 2-oxoacid dehydrogenases and cytosolic-oriented glycerol phosphate dehydrogenase (8). In total, 11 different redox sites have been recognized to generate superoxide in mitochondria (8). Under specific conditions, superoxide formation at the Complex I site $\mathrm{I}_{\text {ubiquinone }}\left(\mathrm{I}_{\mathrm{Q}}\right)$ or Complex III outer $\left(\mathrm{III}_{\mathrm{Qo}}\right)$ site increases with increasing protonmotive force $\Delta p(18,25,29$, $45,57)$. Consequently, the attenuation of mitochondrial superoxide formation by active OXPHOS can occur on the re-entry of protons into the matrix via the $\mathrm{F}_{\mathrm{O}}$ membrane sector of the ATP-synthase (18). Superoxide production also diminishes/rises on the acceleration/retardation of cytochrome $c$ shuttling, respectively (the latter independently of $\Delta p)(21,29,45,50)$, and it depends on ubiquinone homeostasis, or the rate of the reverse electron transport $(8,13,65)$.

Finally, superoxide can be generated at the Complex I flavin $\left(\mathrm{I}_{\mathrm{F}}\right)$ site, which resides close to the flavin-binding site at which $\mathrm{NADH}$ is oxidized $(40,65)$. Here, the increased substrate pressure $S$, defined as the $\mathrm{NADH} / \mathrm{NAD}^{+}$ratio, induces substantial superoxide production $(40,65)$. Since there is an increased substrate load in pancreatic $\beta$ cells on GSIS, faster superoxide production could be predicted. However, the activity of the shuttles mentioned earlier may modulate the Complex I substrate pressure in a manner that is still unpredictable.

Questions of whether superoxide formation increases or decreases on GSIS have already been raised. Opinion was ambiguous. With extensive glucose depletion, the effect of substrate load may overcome the suppressing role of $\mathrm{H}^{+}$returning via the ATP-synthase. Accordingly, increasing mitochondrial ROS on GSIS has been observed $(5,41,56)$. Increasing ATP with decreasing ADP was predicted to diminish mitochondrial ROS formation (23). Indeed, it has been demonstrated for rat pancreatic islets (PIs) that stimulation with glucose reduced the mitochondrial oxidation of roGFP2-Orp1 (15). The superoxide-suppressing role of $\mathrm{H}^{+}$ re-entry via the $\mathrm{F}_{\mathrm{O}}$ sector of the ATP-synthase at a higher intensity of OXPHOS might predominate (37). Also, total reducing equivalents were reported to be increased on GSIS (47). However, the addition of pyruvate alone to PIs increased cellular NADPH without increasing NADH, in contrast to glucose that increased both (53).

To elucidate changes associated with redox homeostasis on GSIS, we have introduced several novel methods to assess nicotinamide nucleotides in the separate compartments of the mitochondrial matrix and cytosol. Moreover, confocal microscopy monitoring with selective fluorescence probes enabled us to study mitochondrial matrix superoxide and $\mathrm{H}_{2} \mathrm{O}_{2}$ generation on the sudden addition of glucose. This approach was validated by using a MitoB probe to monitor ROS accumulation $(14,36,67,68)$. Therefore, by independent means, we provide several lines of unequivocal evidence for a diminished mitochondrial superoxide production on GSIS due to the elevated operation of redox shuttles.

\section{Results}

\section{GSIS increases mitochondrial respiration and membrane potential in INS-E cells}

Unlike with initial $11 \mathrm{~m} M$ glucose, INS-1E cells preincubated with $3 \mathrm{~m} M$ glucose for 2 or $15 \mathrm{~h}$ in cultivation medium responded to the addition of glucose $(25 \mathrm{~m} M$ final concentration) by significantly increasing their respiration in the cultivation medium, phosphorylating/nonphosphorylating respiration ratio ( $R$ r, Fig. 2A, B), parameter $A$ r (Fig. 2C), and mitochondrial inner membrane potential $\Delta \Psi_{\mathrm{m}}$ (Fig. 2D). A strict correlation was found between the ratio $R \mathrm{r}$ and total ATP levels (Fig. 2Ea) or local ATP in the cytosol (excluding ATP signal from insulin granules; Fig. 2Ea, Eb) and mitochondrial matrix ATP (Fig. 2Eb, Ed), assayed by using the respective FRET-based ATeam biosensors. Notably, Rr represents valid estimates of ATP synthesis (OXPHOS activity) required for GSIS (Fig. 2F). In INS-1E cells (Fig. 2B,C) and isolated PIs (Fig. 2Ec), an array of phosphorylating states exists within a sharp hyperbolic increase versus glucose concentration in $\mathrm{Rr}$ (Fig. 2B) (60) or Ar (a fraction of respiration used for ATP synthesis calculated as phosphorylating minus nonphosphorylating respiration normalized to maximum, that is, uncoupled respiration; Fig. 2C). Both were assayed in Krebs-Ringer HEPES buffer containing bovine serum albumin $\left(\mathrm{KRH}^{\mathrm{BSA}}\right)$ after a $1-\mathrm{h}$ preincubation in $\mathrm{KRH}^{\mathrm{BSA}}$ containing $3 \mathrm{~m} M$ glucose. In cells, a steep increase was found between 3 and $8 \mathrm{~m} M$ with the half-maxima of $R \mathrm{r}$ and $A \mathrm{r}$ at $\sim 3.5$ and $4 \mathrm{~m} M$ glucose, respectively, and saturation above $8 \mathrm{~m} M$ glucose (Fig. 2B, C).

Silencing the cytosolic malic enzyme, ME1 insignificantly inhibited respiration at low and high glucose, whereas $\mathrm{H}^{+}$-leak-driven (nonphosphorylating) or maximum (uncoupled) respiration rates were equal (Fig. 2G and Supplementary Fig. S1Aa, Ab). Comparing oxygraph record pairs separately, $R \mathrm{r}$ decreased by $\sim 15 \%$ and $A \mathrm{r}$ by $\sim 25 \%$ (Fig. 2H). Etomoxir, an irreversible inhibitor of carnitine palmitoyltransferase- 1 that blocks fatty-acid $\beta$-oxidation (28), did not affect the $\operatorname{Rr}(A \mathrm{r})$ elevations on transitioning to high glucose (Fig. 2I and Supplementary Fig. S2Aa, Ab). Similarly, inhibiting $\mathrm{Ca}^{2+}$ uptake into mitochondria with ruthenium red had no effect on $R \mathrm{r}$ and $A \mathrm{r}$ (Fig. 2I), neither on respiration (Fig. S2Ba, $\mathrm{Bc}$ ). However, elimination of $\mathrm{Ca}^{2+}$ efflux through the $\mathrm{Ca}^{2+} / \mathrm{Na}^{+}$-antiporter using CGP37157 inhibited phosphorylating respiration (down to $30-40 \%$ ), $\mathrm{H}^{+}$leak-driven respiration, and elevations in ATP synthesis (Fig. 2I and Supplementary Fig. S2Bb, Bc). Silencing of nicotinamide nucleotide transhydrogenase (NNT) in combination with $\Delta \Psi_{\mathrm{m}}$ monitoring suggested the existence of forward NNT mode (NADH-, NADP ${ }^{+}$- plus $\Delta \mathrm{p}$-consuming, producing $\mathrm{NAD}^{+}$and $\mathrm{NADPH}$ ) at both 3 and $25 \mathrm{~m} M$ glucose, since $\Delta \Psi_{\mathrm{m}}$ increased on NNT silencing (Fig. 2L); cf. Santos et al. (58). 
Blockage of PPP with oxythiamine even elevated $R \mathrm{r}$ and $A \mathrm{r}$ with $3 \mathrm{~m} M$ glucose (Fig. 2J). Inhibition of CitC with 1,2,3-benzene-tricarboxylate (BTC) did not significantly affect respiration (Supplementary Fig. $\mathrm{S} 1 \mathrm{Ca}, \mathrm{Cb}$ ), $\mathrm{Rr}$, or Ar (Fig. 2J). Amino-transferase/transaminase inhibitor aminooxyacetic acid (AOA) inhibited respiration by $60 \%$, maximum respiration under $10 \%$, and decreased $R \mathrm{r}$ and Ar (Fig. 2K and Supplementary Fig. S2Ca, Ce). Maximum respiration was inhibited under $14 \%$ (plus the glucoseinduced respiration rise) when glycolysis was blocked with bromopyruvate.

\section{Mitochondrial matrix superoxide release is attenuated on transition to high glucose}

First, we positively validated the method employing the time-lapsed acquisition of MitoSOX Red fluorescence confocal microscopy (Supplementary Fig. S3). Therefore, we can interpret the MitoSOX Red fluorescence elevation to be a genuine measure of the increased superoxide release into the mitochondrial matrix. In INS-1E cells routinely cultured with $11 \mathrm{~m} M$ glucose, there is a nonzero mitochondrial matrix superoxide release (Fig. 3A-D and Supplementary Fig. S3B). Unexpectedly, a rather sharp decrease in superoxide release into the matrix was observed after the addition of glucose (25 mM final) (Fig. 3A and Supplementary Fig. S3G). The corresponding estimates of superoxide release into the matrix $J_{\mathrm{m}}$ decreased and remained low (Fig. 3A, C). The $J_{\mathrm{m}}$, rates represent slopes of increase in integral fluorescence (Fig. 3B and Supplementary Fig. S3G). These values accounted for $\sim 45 \%$ of $J_{\mathrm{m}}$ values before glucose addition (Fig. 3C). Such a drop could not originate from the decreasing $\Delta \Psi_{\mathrm{m}}$, even if MitoSOX Red fluorescence depended on $\Delta \Psi_{\mathrm{m}}$ (which is not the case, see Supplementary Fig. S3). This is because $\Delta \Psi_{\mathrm{m}}$ increases after glucose addition (Fig. 2D) (60). Moreover, the data were qualitatively similar on NNT silencing (Supplementary Fig. S2Dc).

MnSOD (Supplementary Fig. S4A) and total superoxide dismutase (SOD) activities (Supplementary Fig. S4B), measured in parallel, were constant. We also preincubated INS$1 \mathrm{E}$ cells in medium containing only $3 \mathrm{~m} M$ glucose for $15 \mathrm{~h}$ (Fig. 3A, C) or $2 \mathrm{~h}$ (Fig. 3D and Supplementary Fig. S3E-G), which also exhibited diminished $J_{\mathrm{m}}$ after glucose addition (Fig. 3A, C and Supplementary Fig. S3E-G), with a sharp decline between 4 and $9 \mathrm{~m} M$ glucose (Fig. 3D), thus precisely matching glucose-dose dependencies for $R \mathrm{r}$ and $\mathrm{Ar}$ (Fig. 2B, C). This was not observed in completely glucose-depleted cells (Fig. 3A).

To demonstrate the independence of MitoSOX Red responses on the plasma membrane potential $\left(\Delta \Psi_{\mathrm{p}}\right)$, we determined the influence of $\Delta \Psi_{\mathrm{p}}$ changes on $J_{\mathrm{m}}$ rates. Despite having $\Delta \Psi_{\mathrm{p}}$ depolarization induced with glibenclamide

FIG. 2. Respiration, bioenergetic profile, $\Delta \Psi_{\mathrm{m}}$, ATP levels, and insulin secretion in INS-1E cells. (A) Phosphorylating to nonphophosporylating respiration ratio $(R \mathrm{r})$ before (black bars) and after (gray bars) glucose addition to reach $25 \mathrm{~m} M$ final concentration. INS-1E cells were cultured in $11 \mathrm{~m} M$ glucose (" $11 \mathrm{~m} M$ ") or preincubated in cell culture medium with $3 \mathrm{~m} M$ glucose ("3 $\mathrm{m} M$ ") for $15 \mathrm{~h}$. ANOVA $(n=13-15)$ : ** $p<0.05$. (B, C) Glucose-dose dependence of phosphorylating/nonphosphorylating respiratory rate ratio $\operatorname{Rr}(\mathbf{B})$ and the parameter $A \mathrm{r}(\mathbf{C})$. INS-1E cells were preincubated in $\mathrm{KRH}^{\mathrm{BSA}}$ containing $3 \mathrm{~m} M$ glucose for $1 \mathrm{~h}$. Black symbols represent parameters calculated relatively to the respective respiration at $3 \mathrm{~m} M$ glucose, whereas gray symbols at $20 \mathrm{~m} M$ glucose. Endogenous, that is, phosphorylating respiration rates were always recorded before glucose additions to desired concentrations, and after these additions. This was followed by the addition of $1 \mu M$ oligomycin, but oligomycin was also added to $\mathrm{KRH}^{\mathrm{BSA}}$ with $3 \mathrm{~m} M$ glucose in parallel runs. In this way, parameter $R \mathrm{r}$ was calculated for situations before (black symbols) and after glucose addition (gray symbols). FCCP was adjusted (titrated) for maximum response to estimate the maximum (uncoupled) respiration for each glucose concentration, and data were used to calculate the parameter $A \mathrm{r}$ (phosphorylating minus nonphosphorylating respiration rate divided by the maximum respiration rate with FCCP). Student's $t$-test: $* * * p<0.001$. (D, L) Mitochondrial membrane potential $\left(\Delta \Psi_{\mathrm{m}}\right)$ estimated by using TMRE (D) and JC-1 (L). (D) $\Delta \Psi_{\mathrm{m}}$ is plotted against final glucose concentrations as ratios of TMRE fluorescence after $\left(\Delta \Psi_{\mathrm{m}} \mathrm{Glc}\right)$ and before $\left(\Delta \Psi_{\mathrm{m}}\right.$ basal) glucose addition on a logarithmic scale; $(\mathbf{L}) \mathrm{JC}-1$-estimated $\Delta \Psi_{\mathrm{m}}$ is displayed in a diagram for 3 and $25 \mathrm{~m} M$ glucose in cells transfected with a scrambled ("Scrl") siRNA or NNT siRNA. ANOVA $(n=8)$ : ** $p<0.02$. (E) Correlations between ATP levels assayed using bioluminescence (black points) or cytosolic (Ea; red points; red X-axis) and mitochondrial matrix ATeam FRET-sensor (Ed; blue points; blue x-axis) and phosphorylating to nonphophosporylating respiration ratios $R \mathrm{r}$ - INS-1E cells were preincubated at varying glucose in cell culture medium without pyruvate for $2 \mathrm{~h}$ and, subsequently, respiration and ATP levels were measured. Confidence for the correlations was 95\%. (Eb) Ratios of YFP and CFP emission of the ATeam FRET sensors are plotted before and after transition from 3 to $20 \mathrm{~m} M$ glucose (same color coding as above). ANOVA $(n=33-47)$ : *** $p<0.001$. (Ec) Parameter $R$ r for respiration of PIs - assayed by using the Seahorse apparatus, in which irreproducible results are obtained with FCCP, hence Ar cannot be derived. (F) Time courses for insulin secretion. Secreted insulin levels are shown after glucose addition ( $25 \mathrm{~m} M$ final concentration) to INS-1E cells cultured in $11 \mathrm{~m} M$ glucose (black triangles) or preincubated in standard cell culture medium containing $3 \mathrm{~m} M$ glucose for $2 \mathrm{~h}$ (black squares). Gray symbols: time courses without glucose addition. In addition, cells were preincubated in KRH buffer for $5 \mathrm{~min}$ before the ELISA insulin assay in KRH. (G) Effect of ME1 silencing on endogenous respiration rates $(n=6)$ with 3 and $25 \mathrm{mM}$ glucose and nonphosphorylating respiration ("oligomycin"; $1 \mu M$ oligomycin) and maximum respiration ("FCCP," adjusted for maximum response) with $25 \mathrm{~m} M$ glucose; or $(\mathbf{G}-\mathbf{K})$ effects of ME1 silencing and metabolic or transport inhibitors (I-K) on respiratory parameters calculated as described earlier- $R$ r (left y-axis) and $A r$ (right y-axis). For the original respiration data and their replicates, see Supplementary Figures S1 and S2. Ruthenium red $(7 \mu M)$, etomoxir $(100 \mu M), 10 \mu M$ CGP37157, oxythiamine (40 $\mu M)$, BTC $(10 \mathrm{~m} M)$, and AOA $(4 \mathrm{~m} M)$ were present as indicated. ANOVA $(n=4-6)$ : * $p<0.1 ; * * p<0.05$; *** $p<0.001$; or Student's $t$-test for chosen pairs: ${ }^{\# \#} p<0.05 ;{ }^{\# \#} p<0.001$. ANOVA, analysis of variance; AOA, aminooxyacetic acid; BSA, bovine serum albumin; BTC, 1,2,3-benzene-tricarboxylate; FCCP, 4-(trifluoromethoxy)phenylhydrazone; KRH, Krebs-Ringer HEPES buffer; ME1, cytosolic malic enzyme; ns, nonsignificant; PI, pancreatic islet; siRNA, small interfering RNA; TMRE, tetramethylrhodamine ethyl ester. Color images are available online. 
A

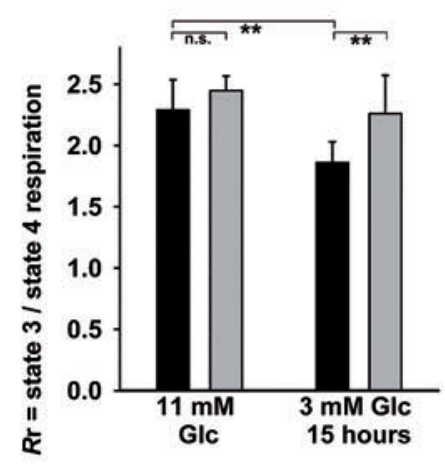

D

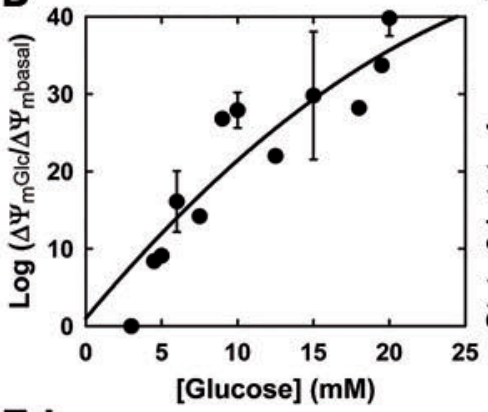

Ed

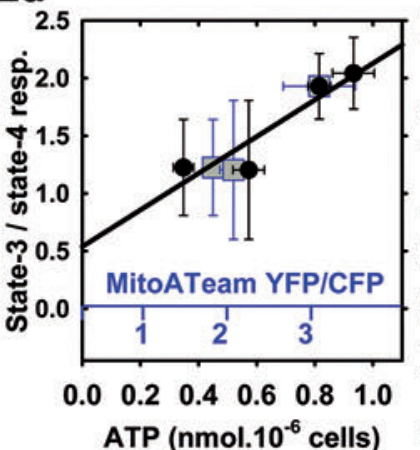

$\mathbf{F}_{30}$

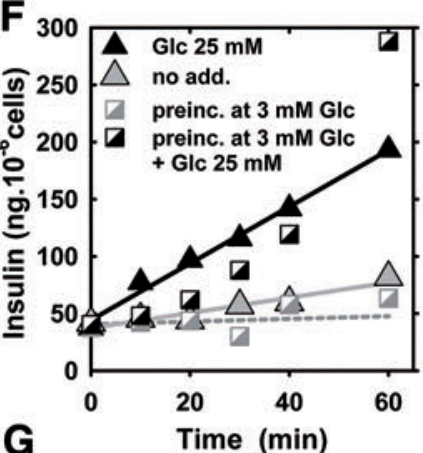

G Time (min)

흥

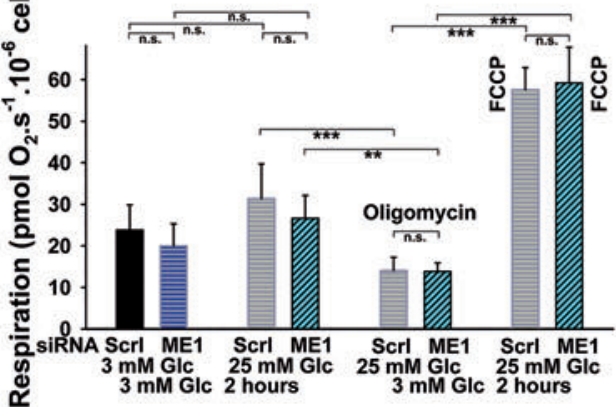

$\mathrm{Ea}$
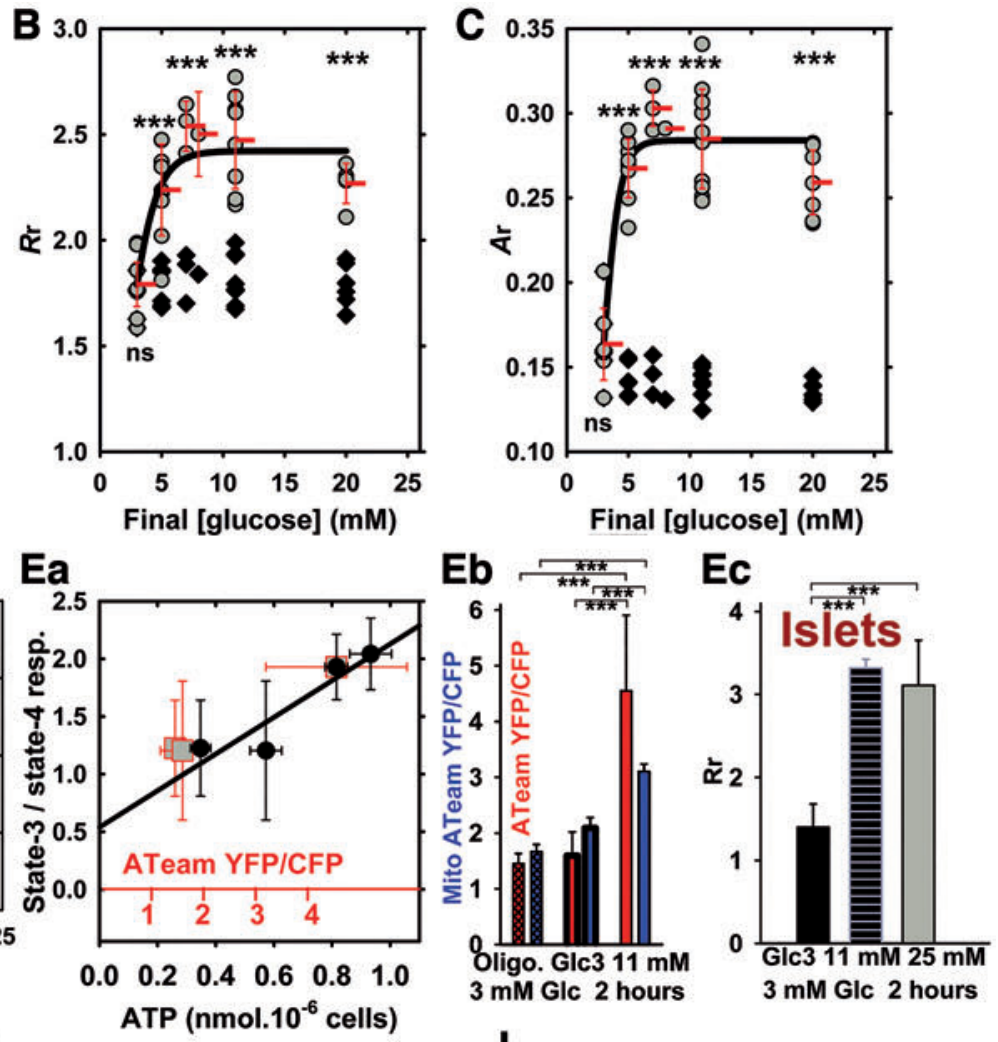

I
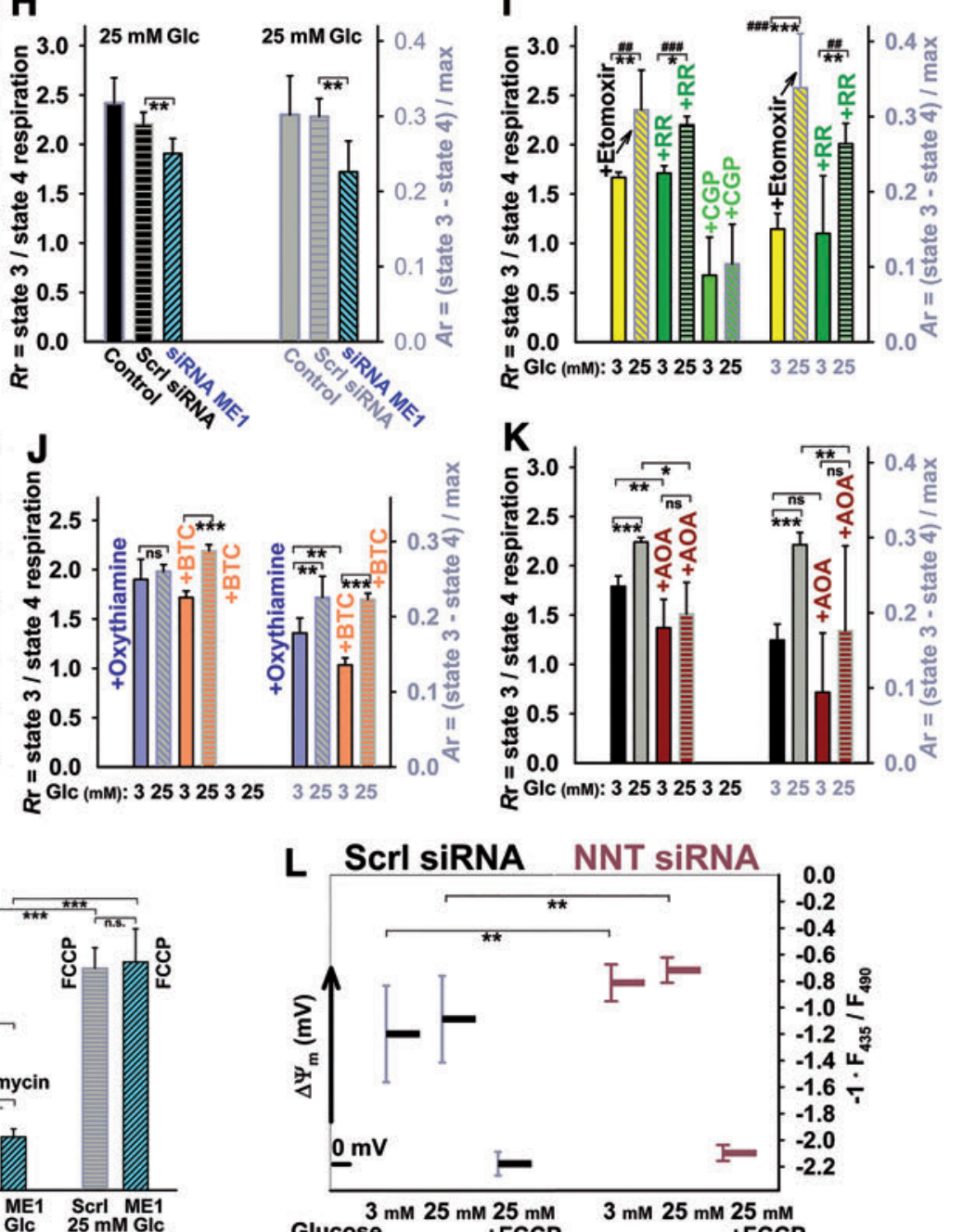

Glucose $25 \mathrm{mM} 25 \mathrm{mM}$ 
before the addition of glucose or blocked with cromakalim, the obtained responses to glucose were equal (Supplementary Fig. S3E, F). The basal $J_{\mathrm{m}}$ rates were on average $1.7 \pm 0.1$ times and 1.2 \pm 0.3 times higher than for INS-1E cells preincubated for $25 \mathrm{~h}$ (Fig. 3A, C) and $2 \mathrm{~h}$ (Supplementary Fig. S3E, F) with $3 \mathrm{~m} M$ glucose, respectively. The additions of $25 \mathrm{~m} M$ glucose caused these $J_{\mathrm{m}}$ rates to drop on average to $40 \%$ of the basal $J_{\mathrm{m}}$ rate (Fig. $3 \mathrm{C}$ ). This corresponds to $44 \%$ of $J_{\mathrm{m}}$ before the addition of glucose to cells cultivated with $11 \mathrm{~m} M$ glucose (denoted as $J^{11}$ mo; Fig. 3C). MnSOD and total SOD activities were constant (Supplementary Fig. S4A, B).

Isolated PIs kept in $5.5 \mathrm{mM}$ glucose exhibited a similar decrease in $J_{\mathrm{m}}$ after glucose supplementation to $25 \mathrm{mM}$ (Fig. 3E). For this assay, confocal monitoring was replaced with fluorimetry, while surveying fast-recorded MitoSOX Red spectra over the time course of the experiment (Supplementary Fig. S3H).

Next, we verified the MitoSOX Red confocal monitoring data by using a MitoB probe and liquid chromatographymass spectrometry (LC-MS) quantification of its oxidized product MitoP (14, 36, 67, 68). Using an identical experimental set-up, but prolonged to $2 \mathrm{~h}$ after the addition of glucose, we clearly found less MitoP formed after high-glucose $(25 \mathrm{mM}) 2$-h treatment when compared with $3 \mathrm{mM}$ glucose. This was reflected by the lower MitoP/ (MitoB+MitoP) ratios in INS-1E cells (Fig. 3F, left panel) and PIs (Fig. 3G, H). Again, similar data were obtained on NNT silencing (Supplementary Fig. S2Dd) and in PIs isolated from NNT-deficient C57BL6/J (Fig. 3G) and NNTnormal C57BL6/N mice (Fig. 3H), evidencing that the NNT absence in INS-1E cells and in C57BL6/J mice (58) does not affect the ROS decline on GSIS. This decline persisted also after $2 \mathrm{~h}$ of incubation with high glucose, when monitored with MitoSOX Red (Supplementary Fig. S5A, B). An entirely opposite response was found when Mito-paraquat was added to elevate oxidative status in the mitochondrial matrix of INS-1E cells (Fig. 3F, right panels). In conclusion, independent verification by LC-MS and MitoB confirmed diminished mitochondrial superoxide formation on GSIS.

Glucose-induced decrease in $J_{\mathrm{m}}$ rates was also observed in the presence of etomoxir (Fig. 4A) (24), AOA (of a lower extent) (Supplementary Fig. S2Cc), and when the malate/ aspartate shuttle (Fig. 1B) (42) should be eliminated by silencing the participating aspartate/glutamate antiporters SLC25A12/AGC1/aralar and SLC25A13/AGC2 or both, independently of glutamine presence (Fig. 4B). Glutamine itself decreased $J_{\mathrm{m}}$ rates, due to the accelerated respiration, and also due to the malate/aspartate shuttle blockage, which decreased $J_{\mathrm{m}}$ rates at $3 \mathrm{~m} M$ glucose down to $55-70 \%$ (Fig. 4B). Similarly, ruthenium red, a blocker of the $\mathrm{Ca}^{2+}$ uniporter (Fig. 4A), and PPP inhibitors (6-aminonicotinamide [6AN] and oxythiamine) (Fig. 4C) elicited a similar outcome.

Analogical patterns were obtained with MitoB (Fig. 4D-F), despite a massively higher $\mathrm{H}_{2} \mathrm{O}_{2}$ accumulation at $3 \mathrm{~m} M$ glucose with etomoxir, ruthenium red, CGP37157, at inhibited PPP, and when omitting glutamine. The declines with etomoxir, at inhibited PPP, and without glutamine were high (Fig. 4D-F). Due to the inhibition of respiration/OXPHOS, the observed decrease in accumulated ROS (and also the onset of oxidative stress with ruthenium red) was relatively nonspecific on blockage of the $\mathrm{Ca}^{2+} / \mathrm{Na}^{+}$efflux with CGP37157 (Fig. 4D). The discrepancy of increasing accumulated ROS versus the MitoSOX assay results could stem from such nonspecific effects. Nevertheless, there was a decrease in the $2 \mathrm{~h}$-accumulated ROS with AOA and when the malate/aspartate shuttle (42) was eliminated by silencing

FIG. 3. Release of superoxide and $\mathrm{H}_{2} \mathrm{O}_{2}$ into the mitochondrial matrix of INS-1E cells on GSIS. INS-1E cells (A-D) were assayed for surplus mitochondrial matrix superoxide release (over MnSOD consumption) by using confocal microscopy monitoring (B) of MitoSOX Red fluorescence (see also Supplementary Fig. S3G). PIs were assayed similarly (E) by using MitoSOX Red spectra scanning. Black columns: values before GSIS; gray columns: values after GSIS. (A) Representative traces - $J_{\mathrm{m}}$ rates were determined from the slopes of linearized traces, such as illustrated, of increasing MitoSOX Red integral fluorescence encompassing mitochondrial ROI, plotted versus time for each series of the corresponding confocal images $(18,28,29)$. When indicated, INS-1E cells were cultured in $11 \mathrm{~m} M$ glucose, or preincubated in medium containing $3 \mathrm{~m} M$ glucose for $15 \mathrm{~h}$. Alternatively, cells were preincubated in KRH medium containing bicarbonate "0 $\mathrm{m} M$." Final glucose levels after a glucose addition are indicated by arrows. (B) Representative confocal images for MitoSOX Red assay carried out separately for 3 and $25 \mathrm{~m} M$ glucose. (C) Quantification of mitochondrial superoxide production rates $J_{\mathrm{m}}$ for cells cultured in medium containing $11 \mathrm{~m} M$ glucose (" $11 \mathrm{~m} M$ Glc") or preincubated in cell culture medium containing $3 \mathrm{~m} M$ glucose (" $3 \mathrm{~m} M$ Glc $15 \mathrm{~h}$ "). $J_{\mathrm{m}}$ rates were normalized to average $J_{\mathrm{m}}$ rates obtained in cells cultured in $11 \mathrm{~m} M$ glucose before GSIS $\left(J^{11}{ }_{\mathrm{m} 0}\right)$. ANOVA $(n=11 ; n=6$ for $3 \mathrm{~m} M 15 \mathrm{~h})$ : $* * * p<0.001$. (D) Decrease in mitochondrial matrix-released superoxide-dose response related to the final glucose concentration ([glucose]) performed by using MitoSOX Red confocal microscopy monitoring. The higher the final glucose concentration reached, the higher the decrease in $J_{\mathrm{m}}$ rates on glucose addition. (E) Isolated mouse PIs: $J_{\mathrm{m}}$ rates before (black column) and 15 min after glucose addition (gray column) were derived from changes in MitoSOX Red spectra after glucose addition to isolated mouse PIs (cf. Supplementary Fig. S3H). Student's $t$-test $(n=3)$ : \#\#\# $p<0.001$. (F-H) MitoP/(MitoB+MitoP) ratios reflecting matrix ROS accumulated after $2 \mathrm{~h}$ in INS-1E cells or PIs isolated from the indicated mouse strains. Ratios were normalized to values obtained for incubations in medium with $3 \mathrm{~m} M$ glucose. Mitoparaquat, $20 \mu M$. ANOVA $(n=4)$ : $* * p<0.05$; *** $p<0.001$. (I-N) Mitochondrial matrix $\mathrm{H}_{2} \mathrm{O}_{2}$ release. $J_{\mathrm{m}}{ }^{\mathrm{H} 2 \mathrm{O} 2}$ rates were assessed by mito-HyPer fluorescence confocal microscopy monitoring, whereas $\mathrm{pH}$ changes were accounted for by mitoSypHer. The differences in these records were taken as being proportional to the net mitochondrial matrix $\mathrm{H}_{2} \mathrm{O}_{2}$ release. (I-K) Representative differential time courses are illustrated for cells preincubated in $3 \mathrm{~m} M$ glucose (I) or cultured in medium with $11 \mathrm{~m} M$ glucose $(\mathbf{J})$, and after the addition of $20 \mu M$ rotenone $(\mathbf{K})$ or $1 \mu M$ S1QEL (L). Relative (M) and approximate absolute $(\mathbf{N})$ quantifications of the resulting $J_{\mathrm{m}}{ }^{\mathrm{H} 2 \mathrm{O} 2}$ rates are shown. ANOVA $(n=3-6)$ : *** $p<0.001$. MnSOD, manganese superoxide dismutase; ROI, regions of interests; ROS, reactive oxygen species; S1QEL, suppressor of complex 1 site $\mathrm{Q}$ electron leak. Color images are available online. 

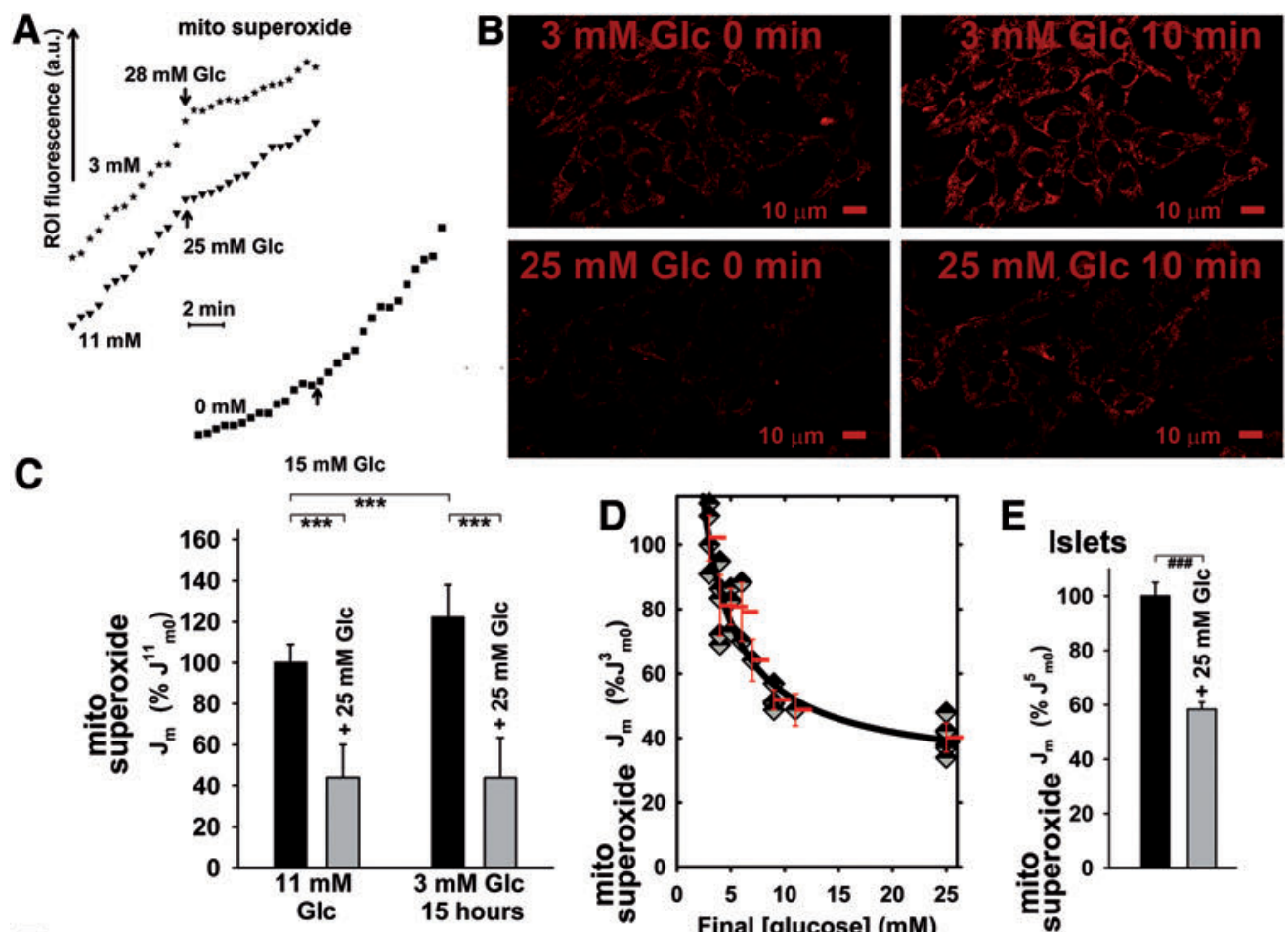

$25 \mathrm{mM}$ Glc 0 min
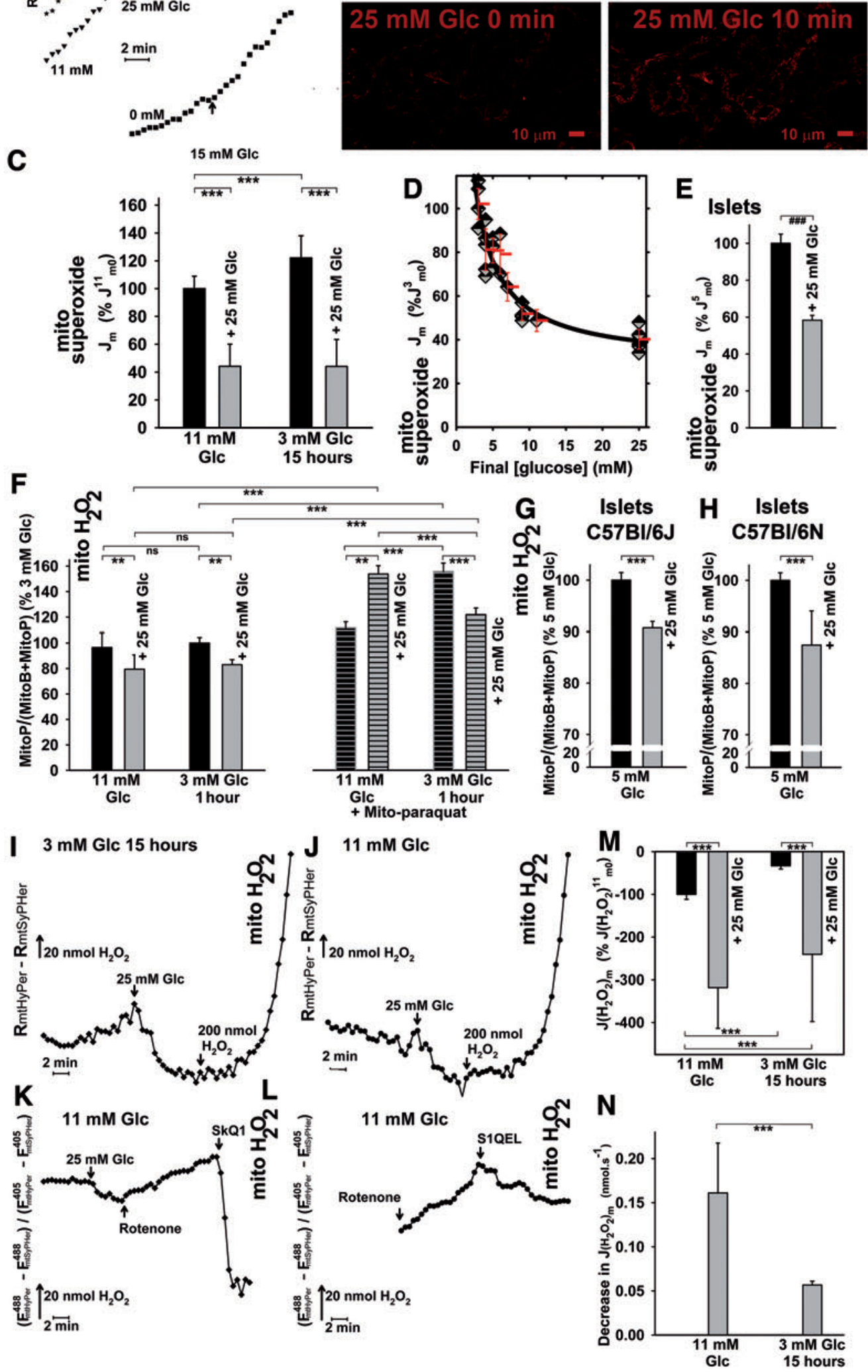
SLC25A12/AGC1/aralar or SLC25A13/AGC2 and both (Fig. 4E and Supplementary Fig. S2Cb, Cd).

\section{Mitochondrial matrix $\mathrm{H}_{2} \mathrm{O}_{2}$ release slows down in INS-1E cells on glucose addition}

Using mito-HyPer, a $\mathrm{H}_{2} \mathrm{O}_{2}$ selective protein-based probe targeted to the mitochondrial matrix (4), we monitored time courses of $\mathrm{H}_{2} \mathrm{O}_{2}$ release into the mitochondrial matrix under identical conditions to the MitoSOX Red monitoring. As expected, the corresponding $J_{\mathrm{m}}{ }^{\mathrm{H} 2 \mathrm{O} 2}$ rates decreased on GSIS, hence independently confirming MitoSOX Red results by a third methodical approach.

To eliminate any possible dependency of mito-HyPer on $\mathrm{pH}$, the $\mathrm{H}_{2} \mathrm{O}_{2}$-insensitive probe mito-SypHer was used in parallel (15). Any interfering contribution of $\mathrm{pH}$ was compensated for by subtracting mito-SypHer from the mitoHyPer fluorescence signal. The signal was calculated as the integral of fluorescence in the regions of interests (ROI) per unit area within the equal area of the mitochondrial network for each of two excitations at 488 and $405 \mathrm{~nm}$. The resulting differential fluorescence data $(\Delta F)$ were used to calculate the $R\left(\mathrm{H}_{2} \mathrm{O}_{2}\right)$ ratios of corrected mito-HyPer emission excited at 488 versus $405 \mathrm{~nm}\left(R\left(\mathrm{H}_{2} \mathrm{O}_{2}\right)=\Delta F^{H_{485}} / \Delta F^{{ }^{H}}{ }_{405}\right)$ (Fig. 3I). Despite having an approximate calibration, that is, since not all of the externally added $\mathrm{H}_{2} \mathrm{O}_{2}$ penetrates into the mitochondrial matrix and influences the probes localized there, we expressed the slopes of the derived dependencies as upper limits to rates of $\mathrm{H}_{2} \mathrm{O}_{2}$ production $J_{\mathrm{m}}{ }^{\mathrm{H} 2 \mathrm{O}}$ in $\mathrm{nmol} \cdot \mathrm{s}^{-1}$.

The main features of the resulting $\mathrm{H}_{2} \mathrm{O}_{2}$ monitoring (Fig. 3I-N) were as follows: In the presence of rotenone, a permanently increased $R\left(\mathrm{H}_{2} \mathrm{O}_{2}\right)$ was observed, giving a certain positive $J_{\mathrm{m}}{ }^{\mathrm{H} 2 \mathrm{O} 2}$ rate and reflecting the $\mathrm{H}_{2} \mathrm{O}_{2}$ release into the mitochondrial matrix (Fig. 3K, L). In contrast, relatively negative $J_{\mathrm{m}}{ }^{\mathrm{H} 2 \mathrm{O} 2}$ rates were obtained for INS-1E cells in 3 (Fig. 3I) and $11 \mathrm{mM}$ glucose (Fig. 3J), thus indicating either an actual $\mathrm{H}_{2} \mathrm{O}_{2}$ efflux from the mitochondrial matrix or alternatively a decrease in $\mathrm{H}_{2} \mathrm{O}_{2}$ formation (the two options being indistinguishable). The subsequent glucose addition ( $25 \mathrm{~m} M$ ) led to a further decrease in $\mathrm{H}_{2} \mathrm{O}_{2}$ release into the mitochondrial matrix (or an increase in $\mathrm{H}_{2} \mathrm{O}_{2}$ efflux, or alternatively both). Consequently, higher negative $J_{\mathrm{m}}{ }^{\mathrm{H} 2 \mathrm{O} 2}$ rates were apparent at $25 \mathrm{mM}$ glucose (Fig. 3M, N). This qualitatively confirms the results obtained by using MitoSOX Red superoxide monitoring (Fig. 3C) or MitoB (Fig. 3F).

Validation of positive/negative $J_{\mathrm{m}}{ }^{\mathrm{H} 2 \mathrm{O} 2}$ rate transitions was done by using mitochondria-specific antioxidants, which prevent rotenone-induced superoxide generation, and hence $\mathrm{H}_{2} \mathrm{O}_{2}$ formation in mitochondria in vivo. Mitochondriatargeted antioxidants, SkQ1 and suppressor of Complex I site $\mathrm{Q}$ electron leak (S1QEL) changed the positive $J_{\mathrm{m}}{ }^{\mathrm{H} 2 \mathrm{O} 2}$ rates into negative ones. These results reflect the powerful antioxidant action of these agents within the mitochondrial matrix (Fig. 3K, L).

\section{Mitochondrial matrix superoxide release at blocked redox shuttles}

Three redox shuttles in pancreatic $\beta$ cells should hypothetically facilitate GSIS by exporting reducing equivalents from the mitochondrial matrix into the cytosol (34), where ME1 or IDH1 produces NADPH (Fig. 1A) (35). It should be noted that two PPP enzymes also form NADPH. Since such export of reducing equivalents should affect matrix redox homeostasis, we evaluated changes in the matrix superoxide release $J_{\mathrm{m}}$ (Fig. $4 \mathrm{H}$ ) and 2-h accumulation of $\mathrm{H}_{2} \mathrm{O}_{2} / \mathrm{ROS}$ in INS-1E cells (Fig. 4I) and PIs (Supplementary Fig. S6) while blocking the redox shuttles by inhibiting citrate transport with BTC (35) (Fig. 4H and Supplementary Figs. S1Ca and S2Dc) and 4-chloro-3-[[(3-nitrophenyl)amino]sulfonyl]benzoic acid (CTH) (Fig. 4I); and also inhibiting 2OGC by $n$-butylmalonate (Fig. 4H, I and Supplementary Fig. S1Da). In the presence of mitochondrial metabolite carrier inhibitors, $J_{\mathrm{m}}$ rates increased (Fig. $4 \mathrm{H}$ ) and $\mathrm{H}_{2} \mathrm{O}_{2} / \mathrm{ROS}$ accumulation did not significantly change or, in some instances, even increased on glucose addition (Fig. 4I and Supplementary Fig. S6).

INS-1E cells with silenced ME1 (or ME1 plus CitC) exhibited much higher $J_{\mathrm{m}}$ rates at 3 or $11 \mathrm{~m} M$ glucose, which were less retarded or were increased on glucose addition $(25 \mathrm{mM})$ when compared with samples with scrambled small interfering RNA (siRNA) (Fig. 4J and Supplementary Fig. S1Ab). This was confirmed by MitoB (Fig. 4K, L). Glucose-induced decreases in 2-h accumulation of $\mathrm{H}_{2} \mathrm{O}_{2} /$ ROS ceased with ME1 silencing (Fig. 4K, L). Thus, the redox shuttle turnover was retarded, due to the blockage of key components by silencing (90\% of ME1 transcript vanished, and $60 \%$ of CitC transcript when silenced simultaneously, or 90\% when alone; Supplementary Fig. S1Ab). The slower metabolic turnover resulted in higher superoxide release to the mitochondrial matrix. Similarly, the silencing of IDH2 allowed by $\sim 20 \%$ lower decline of $J_{\mathrm{m}}$ rates on transition from 3 to $25 \mathrm{~m} M$ glucose (Supplementary Fig. S2Eb, Ec) reflected the shutdown of only one among the three shuttles (Supplementary Fig. S2Ea). In MitoB

FIG. 4. MitoSOX Red and MitoB responses in the presence of various inhibitors of carriers and metabolism, on silencing ME1, pyruvate carboxylase, or aspartate/glutamate carriers of the malate/aspartate shuttle. Mitochondrial matrix superoxide release rates $J_{\mathrm{m}}(\mathbf{A}-\mathbf{C}, \mathbf{H}, \mathbf{J})$ and changes in $2 \mathrm{~h}$-accumulation of $\mathrm{H}_{2} \mathrm{O}_{2} / \mathrm{ROS}(\mathbf{D}-\mathbf{G}, \mathbf{I}, \mathbf{K}, \mathbf{L})$ on transition between 3 and $25 \mathrm{~m} M$ glucose: (A, D) in the presence of RR (green) $(7 \mu M)$, etomoxir $(100 \mu M)$ (yellow bars), and CGP37157 (10 $\mu M$ ) (light green bar); (B, E) on blockage of components of the malate/aspartate shuttle (dark red bars), that is, silencing of aspartate/glutamate antiporters SLC25A12/AGC1/aralar and SLC25A13/AGC2. (C, F) PPP blockage using $1 \mathrm{~m} M$ 6AN or $40 \mu M$ oxythiamine (blue bars); (C, G) pyruvate carboxylase silencing (red/orange bars); for scrambled siRNA cf. ( J, K); (H, I) assays with CitC (10 $\mathrm{mM}$ BTC; orange bars) and 2OGC inhibitor (5 $\mathrm{m} M n$-butylmalonate; yellowgreen bars); and ( J-L) assays on silencing cytosolic malic enzyme ("siRNA ME1") (dark blue or aquamarine bars) alone or together with CitC ("si ME1+ si CitC") as compared with scrambled siRNA (gray bars). $J_{\mathrm{m}}$ rates obtained in the presence of $25 \mathrm{~m} M$ glucose $\left(J^{25}{ }_{\mathrm{m}}\right)$ were normalized either to rates obtained in $11 \mathrm{~m} M$ glucose before GSIS $\left(J^{11}{ }_{\mathrm{m} 0}\right)$, rates obtained in $3 \mathrm{mM}$ glucose before GSIS $\left(J^{3} \mathrm{~m} 0\right)$, or their respective initial glucose concentration before glucose addition $\left(J_{\mathrm{m}}\right.$ before Glc addition $)$. ROS accumulation was normalized to $3 \mathrm{~m} M$ glucose. All preincubations in $3 \mathrm{~m} M$ glucose before the assay were performed for $2 \mathrm{~h}$. ANOVA $(n=3-6)$ : $* * p<0.05$; $* * *<<0.001$; Student's $t$-test: ${ }^{\# \# \# ~} p<0.001$. 6AN, 6aminonicotinamide; PPP, pentose-phosphate pathway; RR, ruthenium red. Color images are available online. 

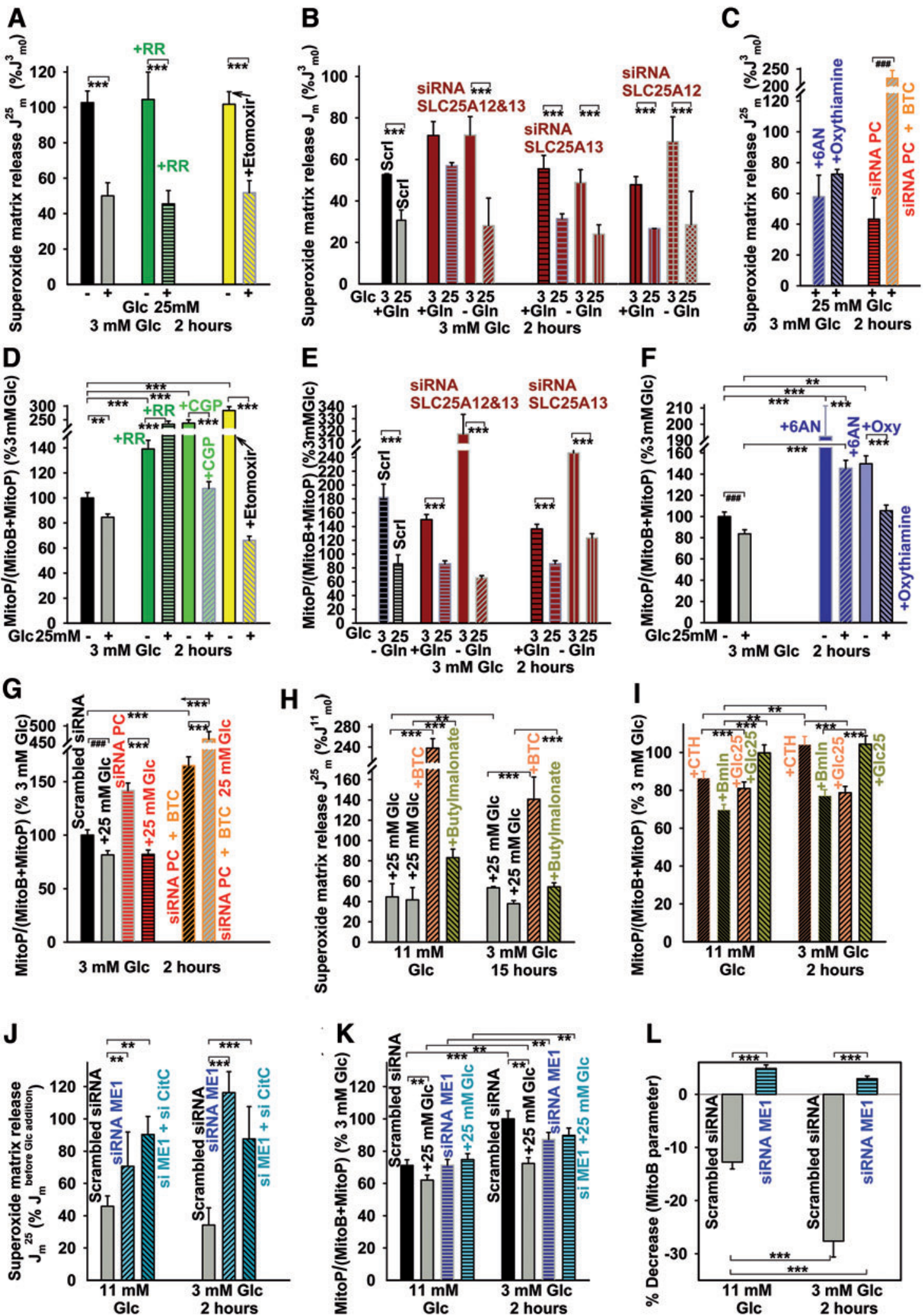

response, analogical decline vanished with IDH2 silencing (Supplementary Fig. S2Ed).

In INS-1E cells with silenced pyruvate carboxylase, the decline in $J_{\mathrm{m}}$ rates was unchanged, but with the simultaneous inhibition of CitC using BTC, the mitochondrial matrix su- peroxide release doubled (Fig. 4C and Supplementary Fig. S1Ba, Bb). Also the decline in accumulated $\mathrm{H}_{2} \mathrm{O}_{2} / \mathrm{ROS}$ was more pronounced, but the further addition of BTC again led to a high oxidative stress at low and high glucose, which was much higher for the latter (Fig. 4G). 
Mitochondrial redox shuttles are responsible for the increase in cytosolic NADPH and matrix $N A D^{+}$ on GSIS

An enzyme-based assay of the total cell NADPH confirmed an increase $(>10 \%)$ in total NADPH on glucose being set to $25 \mathrm{~m} M$ in INS-1E cells (preincubated with 11 or $3 \mathrm{mM}$ glucose; Fig. 5A). This increase was prevented by CitC inhibition with BTC (Fig. 5A) or CTH; and by the 2OGC inhibitor $n$-butylmalonate (Fig. 5A). Parallel confocal fluorescence monitoring of free cytosolic $\mathrm{NADPH}_{\mathrm{F}}$, using the iNAP1 fluorescence probe (Fig. 5B-D; as compared with
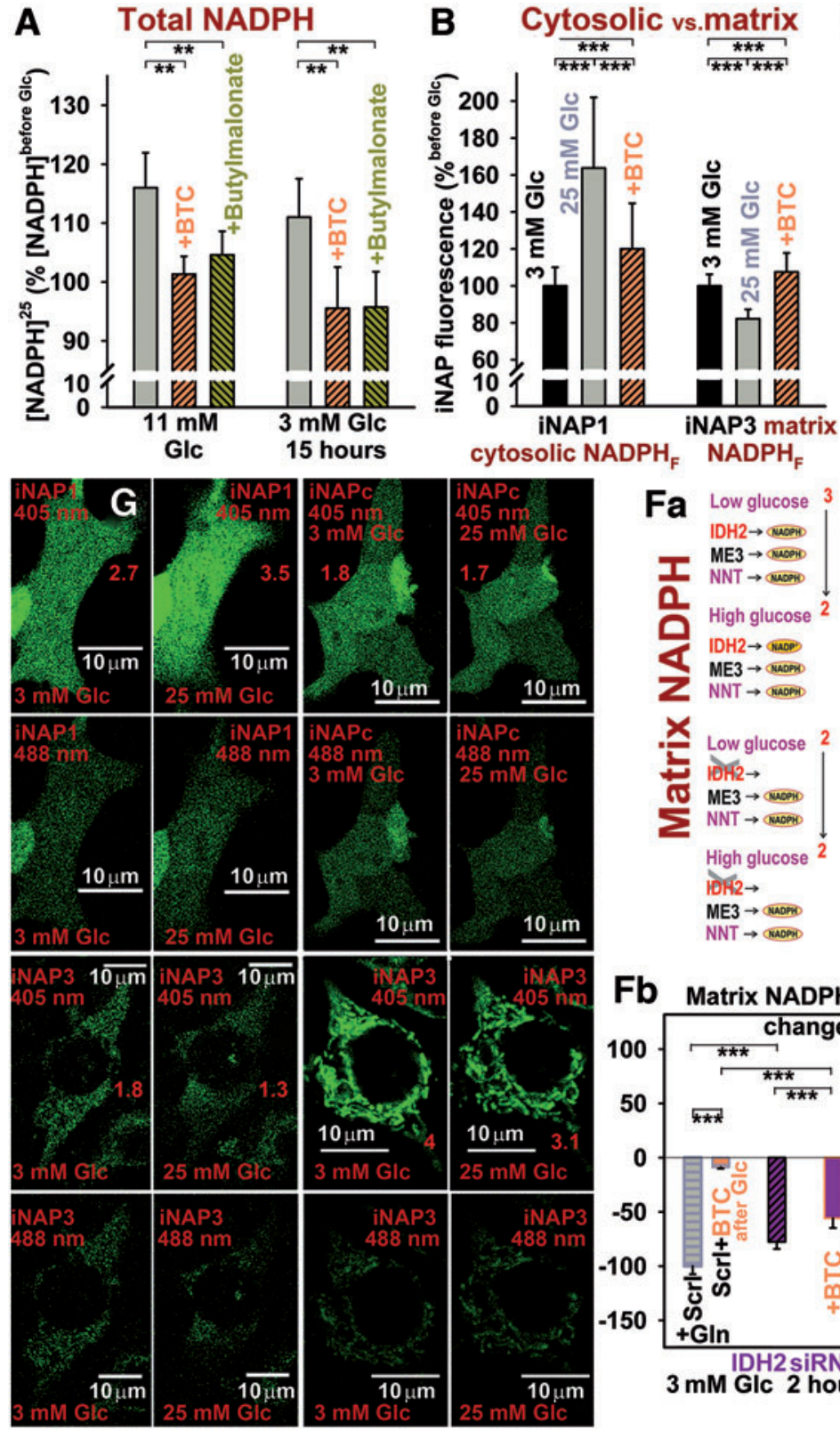

C
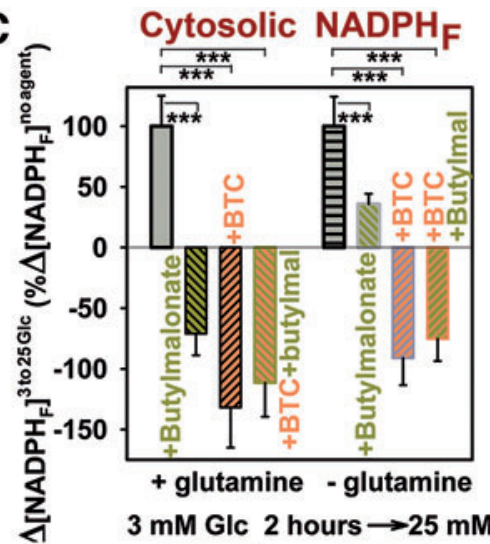

$3 \mathrm{mM}$ Glc 2 hours $\rightarrow 25 \mathrm{mM}$

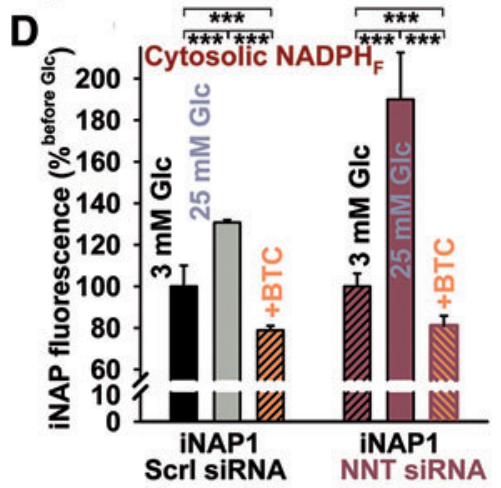

DH2 $\rightarrow$

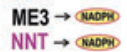
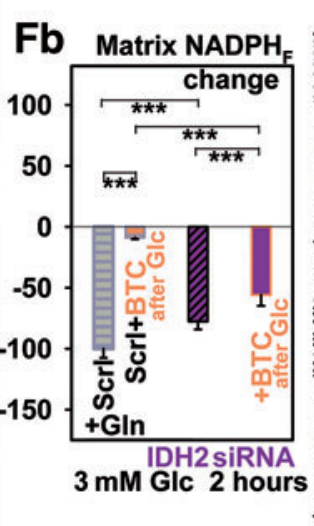

FIG. 5. Cytosolic NADPH elevation on GSIS. (A) Total cell NADPH was assayed by the BioVision kit, ANOVA $(n=3-6) * * p<0.05$; (B-D, G) cytosolic NADPH elevations, or (E-G) NADPH declines within the mitochondrial matrix, assayed by the iNAP1 or iNAP3 fluorescence probes, respectively, in INS-1E cells (preincubated with $3 \mathrm{~m} M$ glucose for $2 \mathrm{~h}$ ) on glucose addition to the final concentration of $25 \mathrm{mM}$; in the absence or presence of CitC inhibitor (10 $\mathrm{m} M$ BTC, orange bars) or 2OGC inhibitor ( $5 \mathrm{~m} M n$-butylmalonate, yellow-green bars) or both; or in NNT-silenced (brown bars, D, E) or IDH2-silenced cells (purple bars, Fb). Inhibitors were added after the glucose addition (B-D). Images in (G) show representative cells transfected with iNAP1 and iNAP3, respectively, or nonresponding iNAPc observed by confocal microscopy at 405 and $488 \mathrm{~nm}$ excitations. Emission ratios 405/488 are indicated by numbers. (Fa) The most probable enzyme contribution to the mitochondrial matrix NADPH pool, under the assumption that NNT always acts in its forward mode (combinations of all NNT possible modes see Supplementary Fig. S2Ef) and of equal enzyme contribution to the

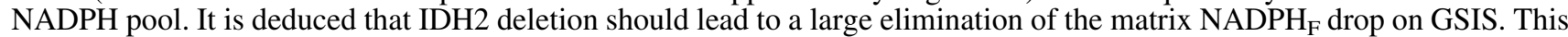
trend was observed only for IDH2-silenced cells with BTC. ANOVA for $(\mathbf{B}, \mathbf{C}) n=40-60$ image spots or $(\mathbf{F b}, \mathbf{B}) n=35-55$; $n=85$ for controls: $* * * p<0.001$. Color images are available online. 
the insensitive iNAPc probe, see Fig. 5G), indicated an $\left[\mathrm{NADPH}_{\mathrm{F}}\right]_{\mathrm{c}}$ increase on transition from 3 to $25 \mathrm{mM}$ glucose. These changes were prevented or, in some instances, reversed by BTC and $n$-butylmalonate (Fig. 5B-D and Supplementary Fig. S7). Thus, the active redox shuttles involving CitC and 2OGC provide the export of reducing equivalents from the mitochondrial matrix (Fig. 1A). The data were similar on NNT silencing (Fig. 5D), confirming that the redox shuttles act upstream of NNT. IDH2 silencing insignificantly inhibited the $\left[\mathrm{NADPH}_{\mathrm{F}}\right]_{\mathrm{c}}$ elevation (Supplementary Fig. S2Ee), since the two remaining shuttles should be still operating, unlike with BTC in IDH2-silenced cells, which left only one, thus preventing the cytosolic $\left[\mathrm{NADPH}_{\mathrm{F}}\right]_{\mathrm{c}}$ elevations.

Simultaneously, the matrix-addressed iNAP3 indicated a decrease in mitochondrial $\left[\mathrm{NADPH}_{\mathrm{F}}\right]_{\mathrm{m}}$ on GSIS (Fig. 5E-G). The matrix NADPH/NADP ${ }^{+}$homeostasis stems mainly from the complex contribution of NNT, IDH2, and the NADP ${ }^{+}$ dependent malic enzyme ME3 (Supplementary Fig. S2Da, $\mathrm{Ea}, \mathrm{Ef})$. However, the iNAP3-monitored drop in $\left[\mathrm{NADPH}_{\mathrm{F}}\right]_{\mathrm{m}}$ was not affected on NNT silencing (Fig. 5E). Figure 5Fa and the simplified S2Ef schematics explain why the matrix $\left[\mathrm{NADPH}_{\mathrm{F}}\right]_{\mathrm{m}}$ should not decline on IDH2 silencing. However, we observed only a lesser decline (high decline with BTC; Fig. 5Fb), resulting from an unequal contribution of the considered enzymes.

\section{Accumulation of selected metabolites and citrate/isocitrate export on GSIS}

Among Krebs cycle metabolites, citrate, malate, fumarate, and oxaloacetate (OAA) were increased at 20 versus $3 \mathrm{mM}$ glucose (Fig. 6A). Metabolite accumulation reflects either an increase in their supply or a decrease in their utilization $(61,62)$. An unchanged malate/fumarate ratio could indicate proportional increases in respiration and turnover of this Krebs cycle segment (Fig. 6B). The decreasing citrate/ pyruvate ratio reflects more rapid pyruvate utilization with $20 \mathrm{~m} M$ glucose. The elevated OAA/pyruvate ratio at the decreasing citrate/OAA ratio supports the increasing pyruvate carboxylase reaction and citrate synthase reaction on GSIS. Also, 2-oxoglutarate (2OG) increased on GSIS, despite being present at lower concentrations due to a high turnover. Estimation of $\beta$-hydroxybutyrate $(\beta$-OHB) to acetoacetate ratio indicated its insignificant small rise on GSIS (Fig. 6C). The observed $\beta$-OHB elevation supports the matrix $\mathrm{NAD}^{+}$increase (Supplementary Fig. S2Fa) $(43,46)$, since the rat $\beta$ OHB dehydrogenase exists only in the matrix.

The existence of ${ }^{13} \mathrm{C}$-incorporation, from $1-{ }^{13} \mathrm{C}$-glutamine into ${ }^{13} \mathrm{C}$-citrate, ${ }^{13} \mathrm{C}$-2OG, or ${ }^{13} \mathrm{C}$-malate, evidenced the operation of the isocitrate/pyruvate shuttle, specifically the reductive carboxylation reaction of IDH2 (Fig. 6D) (59). Despite quantifications of ${ }^{13} \mathrm{C}$-labeled metabolites in INS-1E cells after a 2-h incubation having lower resolution versus those performed after $6 \mathrm{~h}(59)$, we detected about a 1.2-fold rise of ${ }^{13} \mathrm{C}$-incorporation into ${ }^{13} \mathrm{C}$-citrate and a 1.1 -fold rise of ${ }^{13} \mathrm{C}$-malate from $1-{ }^{13} \mathrm{C}$-glutamine, when comparing 3 versus $20 \mathrm{~m} M$ glucose. Here, the increased ${ }^{13} \mathrm{C}$-incorporation represents a higher reaction turnover, since initially, only the natural ${ }^{13} \mathrm{C}$-content exists in analyzed metabolites. The latter was actually subtracted from the measured data of Figure 6D.

\section{Two-channel fluorescence lifetime imaging microscopy-assessed NADPH and NADH or $N A D^{+}$responses to glucose}

We also attempted to evaluate changes in the mitochondrial matrix $\mathrm{NADH}$ or $\mathrm{NAD}^{+}$and trends in changes of $\mathrm{NADH} / \mathrm{NAD}^{+}$ratios by using our novel two-channel fluorescence lifetime imaging microscopy (2chFLIM) method (Figs. 7 and 8), which is able to assess these changes without the need of precise quantifications of separate NADH and $\mathrm{NAD}^{+}$concentrations. In this way, we independently tested our hypothesis that the mitochondrial matrix NADH/NAD ${ }^{+}$ ratio decreases when the redox shuttles (Fig. 1A) are active (Fig. 8B-E). At first, using the quantification developed by Duchen and colleagues (6), we found that on transitions from 3 or 11 to $25 \mathrm{mM}$ glucose, the ratios of bound species $\mathrm{NADPH}_{\mathrm{B}} / \mathrm{NADH}_{\mathrm{B}}$ derived from the $2 \mathrm{chFLIM}$ data within chosen ROI did not significantly change in the matrix (Supplementary Fig. S8) and slightly increased in the cytosol (Fig. 8A). Unlike in the nucleus, the cytosolic $\mathrm{NADPH}_{\mathrm{B}} /$ $\mathrm{NADH}_{\mathrm{B}}$ ratio decreased on the inhibition of CitC (Fig. 8A).

We then extended Duchen's method to estimate the concentration of free mitochondrial NADH $\left([\mathrm{NADH}]_{\mathrm{F}}\right)$ based on the Scatchard equation and the assumption of the predominant participation of Complex I. Consequently, $[\mathrm{NADH}]_{\mathrm{F}}$ was approximated according to eq. $\{10\}$ from the corresponding decay coefficient $v_{\mathrm{F}}$ (Fig. 8B) and the initial $\mathrm{NAD}(\mathrm{P}) \mathrm{H}$ autofluorescence intensity, whereas changes after glucose addition were calculated according to eq. $\{11\}$ (Fig. $8 \mathrm{C}-\mathrm{E}$ ). These estimates showed that $[\mathrm{NADH}]_{\mathrm{F}}$ decreased on average by $20 \%$ on GSIS (Fig. 8C-E). The coefficient $v_{\mathrm{F}}$ itself declined on average by $12 \%$ (Fig. 8B). The decrease ceased or changed to an increase in $[\mathrm{NADH}]_{\mathrm{F}}$ when the citrate export was inhibited with BTC or CTH (Fig. 8C, D) and when ME1 was silenced (Fig. 8E), that is, when blocking at least two redox shuttles.

A faster decay of FAD autofluorescence on GSIS in INS$1 \mathrm{E}$ cells was indicated by shorter lifetimes of both bound FAD (a short $\tau_{1}{ }^{\mathrm{FAD}}=\tau_{\mathrm{BAD}}$ ) and free FAD (a longer $\left.\tau_{2}{ }^{\mathrm{FAD}}=\tau_{\mathrm{FAD}}^{\mathrm{FA}}\right)(\mathrm{Fig} .8 \mathrm{~F})$. This could indicate the existence of the augmented quenching of $\mathrm{FAD}$ by $\mathrm{NAD}^{+}$, thus indicating an $\mathrm{NAD}^{+}$increase. Assuming $\mathrm{NAD}^{+}$in $500-800 \mu M$, the obtained $\tau_{2}{ }^{\text {FAD }}$ values fit into the 2chFLIM calibration performed in permeabilized cells (Fig. 7G, H). Trends in $\left[\mathrm{NAD}^{+}\right]_{\mathrm{F}}$ changes are summarized in Figure $8 \mathrm{~F}$ and $\mathrm{G}$. In contrast, when the citrate export was inhibited with BTC $(\mathrm{CTH})$ or ME1 was silenced, both lifetimes of FAD emission either did not significantly change or even increased (Fig. 8F). The latter indicates a partial loss of quenching and could be ascribed to lower $\left[\mathrm{NAD}^{+}\right]_{\mathrm{F}}$ (Fig. 8G). Otherwise, observations of a rise in mitochondrial matrix $\mathrm{NAD}^{+}$on GSIS further support our Hypothesis I, that the active redox shuttles effectively increase mitochondrial $\mathrm{NAD}^{+}$, not allowing the extensive IDH3 and MDH forward reactions. This leads to the accumulation of matrix $\mathrm{NAD}^{+}$. Obviously, the inhibition of citrate/isocitrate export re-accelerates IDH3, and hence the $\mathrm{NAD}^{+}$increase ceases. Similarly, the pyruvate/malate and the pyruvate/citrate redox shuttle (requiring also CitC) are dependent on the ME1 reaction, as the ME1 silencing causes the disappearance of the observed changes on GSIS.

Despite several simplifications introduced into our calculations of separate concentrations of nicotinamide 

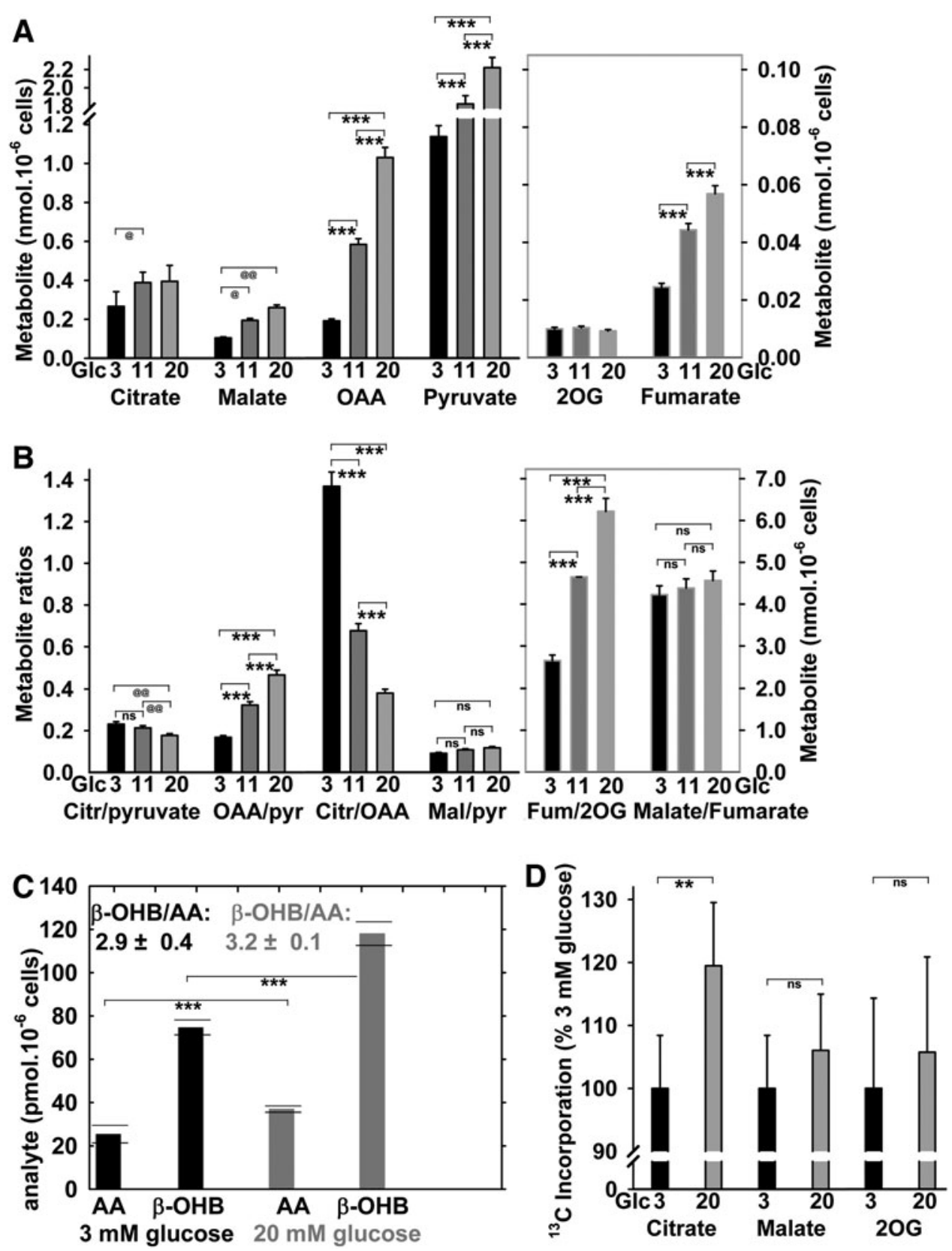

FIG. 6. Targeted metabolomics of the Krebs cycle intermediates, acetoacetate, and $\beta$-OHB and evidence for the isocitrate/pyruvate redox shuttle. Total cellular metabolite levels $(\mathbf{A})$, their selected ratios $(\mathbf{B})$, and $\mathrm{AA}$ and $\beta$-OHB and their ratios as indicated $(\mathbf{C})$ were quantified in INS-1E cells preincubated with $3 \mathrm{~m} M$ glucose for $1 \mathrm{~h}$; then, glucose was raised by zero, 8 , and $17 \mathrm{~m} M$ surplus to reach a final concentration of $3 \mathrm{~m} M$ (black bars), $11 \mathrm{~m} M$ (dark gray bars), and $20 \mathrm{~m} M$ (gray bars) glucose and subsequently incubated for another $30 \mathrm{~min}$. ANOVA for $(\mathbf{A}, \mathbf{B})$ yielded $(n=5) * * * p<0.001$; whereas Student's $t$-test for $(\mathbf{A}, \mathbf{B})$ yielded $(n=5)$ : ${ }^{@} @ p<0.05 ;{ }^{@} p<0.1$; and for $(\mathbf{C})$ (all estimates from two independent experiments are shown with averages and SDs); $p<0.001$ for all combinations between the two compounds. The difference between the $\beta$-OHB/AA ratios was not significant. Notably, the significant $\beta$-OHB increase suggests also the increase in mitochondrial matrix $\mathrm{NAD}^{+}$, since $\beta$-OHB dehydrogenase, which exists only in the mitochondrial matrix, produces $\beta$-OHB from AA at the expense of $\mathrm{NADH}$, thus forming $\mathrm{NAD}^{+}(46)$. The fact that AA does not proportionally decrease reflects other reactions $(43,46)$ and penetration of AA into the cytosol during the sample preparation (Supplementary Fig. S2Fa). (D) ${ }^{13} \mathrm{C}$ incorporation from $1-{ }^{13} \mathrm{C}$-L-glutamine into citrate, malate, and $2 \mathrm{OG}$ is expressed for normalized data for $25 \mathrm{~m} M$ glucose (2-h incubations) in relation to average values obtained after a 2-h incubation with $3 \mathrm{~m} M$ glucose. Data were first calculated in $\%$ of ${ }^{13} \mathrm{C}$ accumulated amounts versus total $\left({ }^{13} \mathrm{C}+{ }^{12} \mathrm{C}\right)$ amount of a given compound when accounted for the natural ${ }^{13} \mathrm{C}$ content. Evidence for the isocitrate/pyruvate redox shuttle is suggested by the existence of the ${ }^{13} \mathrm{C}$-accumulation, as such. This is because the ${ }^{13} \mathrm{C}$-accumulation into citrate from $1-{ }^{13} \mathrm{C}$-glutamine cannot exist on the forward Krebs cycle, since ${ }^{13} \mathrm{C}-\mathrm{CO}_{2}$ is formed and eliminated from the sample; hence any ${ }^{13} \mathrm{C}$-labeled citrate or malate molecule (when subtracting those with naturally occurring ${ }^{13} \mathrm{C}$ ) must originate from the reverse Krebs cycle direction, given by the IDH2mediated NADPH-driven reductive carboxylation of 2OG. ANOVA for (D) $(n=6)$ : $* *<0.05$. Also, insignificantly ("ns") increased ${ }^{13} \mathrm{C}$ incorporation into malate and $2 \mathrm{OG}$ is indicated. $\beta$-OHB, $\beta$-hydroxybutyrate; AA, acetoacetate; ns, nonsignificant; SDs, standard deviations. 


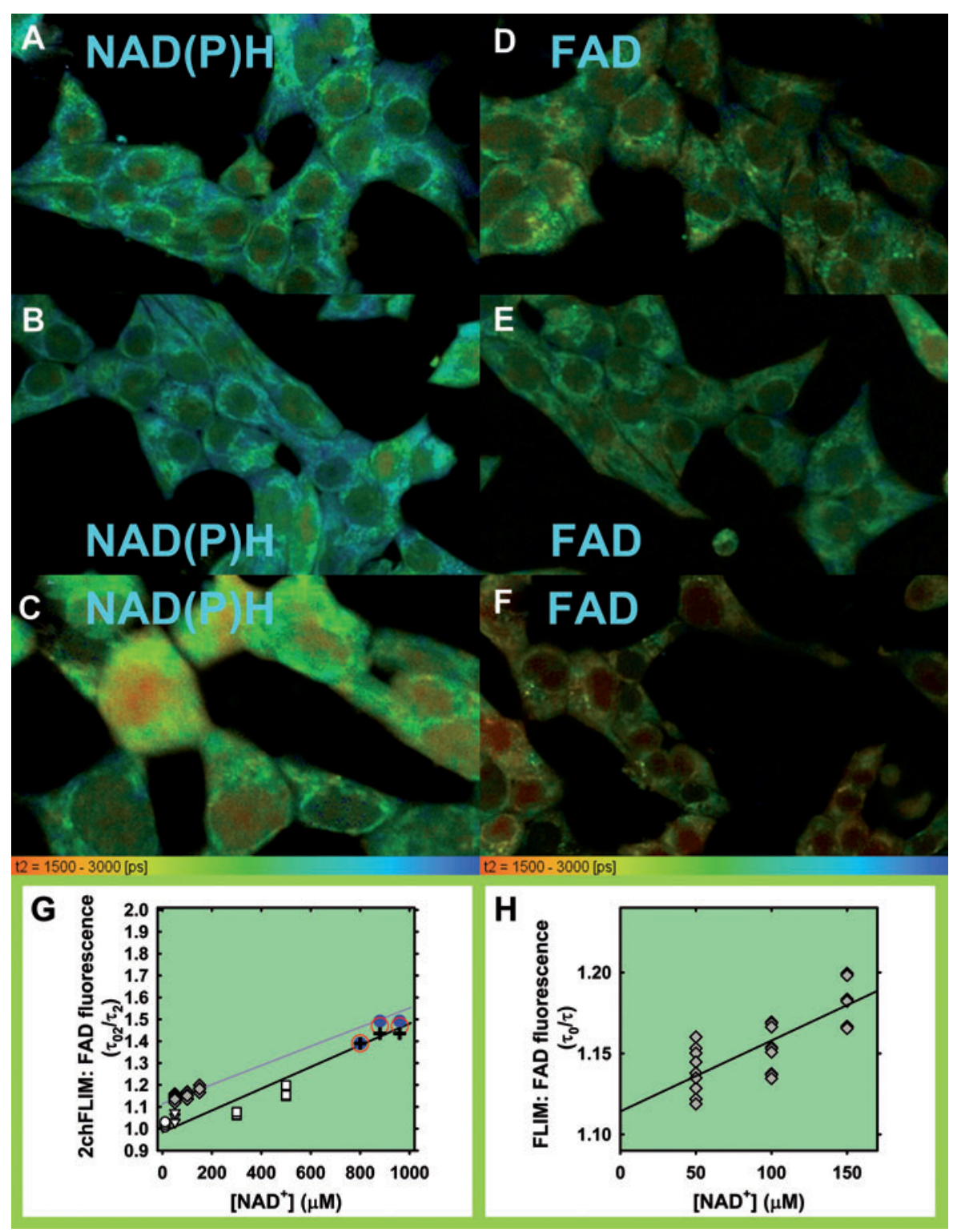

FIG. 7. 2chFLIM images of $\tau_{2}$ mode for bound NAD(P)H and free FAD signals and approximate calibration. (A, D) INS-1E cells cultured in medium with $11 \mathrm{~m} M$ glucose ("Glc11"); (B, E) after glucose addition to reach a final concentration of $25 \mathrm{mM}$ ("Glc25"); (C, F) as (B, E) with $10 \mathrm{mM}$ BTC. (G, H) Approximate calibration. Decreases in singlecomponent resolved FAD emission decay (details in $\mathbf{H}$ ), expressed by the linearized Stern-Volmer relationship (eq. $\{12\}$ ) when $1 \mathrm{~m} M$ FAD in glycerol was titrated by using $50 \mu M$ NAD ${ }^{+}$aliquots (gray data and gray fit). A single decay component $\tau_{0}$ was estimated in the absence of quencher $\left(\mathrm{NAD}^{+}\right)$, whereas component $\tau$ corresponded to the studied $\mathrm{NAD}^{+}$additions (H). Data in (G) (except of gray symbols) show the $\tau 0_{2}{ }^{\mathrm{FAD}}$ from the FAD channel of 2chFLIM relatively to the decreasing $\tau_{2}{ }^{\mathrm{FAD}}$. INS-1E cells were permeabilized by using digitonin $(0.5-1 \mu \mathrm{g})$, whereas we measured and analyzed the FAD emission of either (i) natural cytosolic FAD content (circles, triangles); or (ii) added FAD (10 or $100 \mu M$; squares). Parameter $\tau 0_{2}{ }^{\text {FAD }}$ was estimated first, before addition of $\mathrm{NAD}^{+}$aliquots. Blue or red circles and black crosses: experimental data from Figure 8F: Blue circles-maximum acquired shift in $\tau_{2}{ }^{\mathrm{FAD}}$ on GSIS; red circles - an average shift in $\tau_{2}{ }^{\mathrm{FAD}}$, both from the initial $11 \mathrm{~m} M$ glucose; black crosses - an average shift in $\tau_{2}{ }^{\mathrm{FAD}}$ from the initial $3 \mathrm{~m} M$ glucose. Shifts in $\mathrm{x}$-axis show $10 \%$ and $20 \%$ elevations in $\mathrm{NAD}^{+}$concentration. The data approximately fit to the Stern-Volmer relationship for dynamic quenching (eq. $\{12\}$ ), to which also our experimental $\tau_{2}{ }^{\text {FAD }}$ data (lifetimes for free FAD) from Figure $8 \mathrm{~F}$ fit well under the assumption that mitochondrial matrix $\mathrm{NAD}^{+}$is around $800 \mu \mathrm{M}$ and was $10 \%$ or $20 \%$ elevated on GSIS. Note also that if this $\mathrm{NAD}^{+}$was overestimated and may be lower around $500 \mu \mathrm{M}$, a slightly different Stern-Volmer relationship (eq. $\{12\})$ still would be valid. 2chFLIM, two-channel fluorescence lifetime imaging microscopy. Color images are available online. 

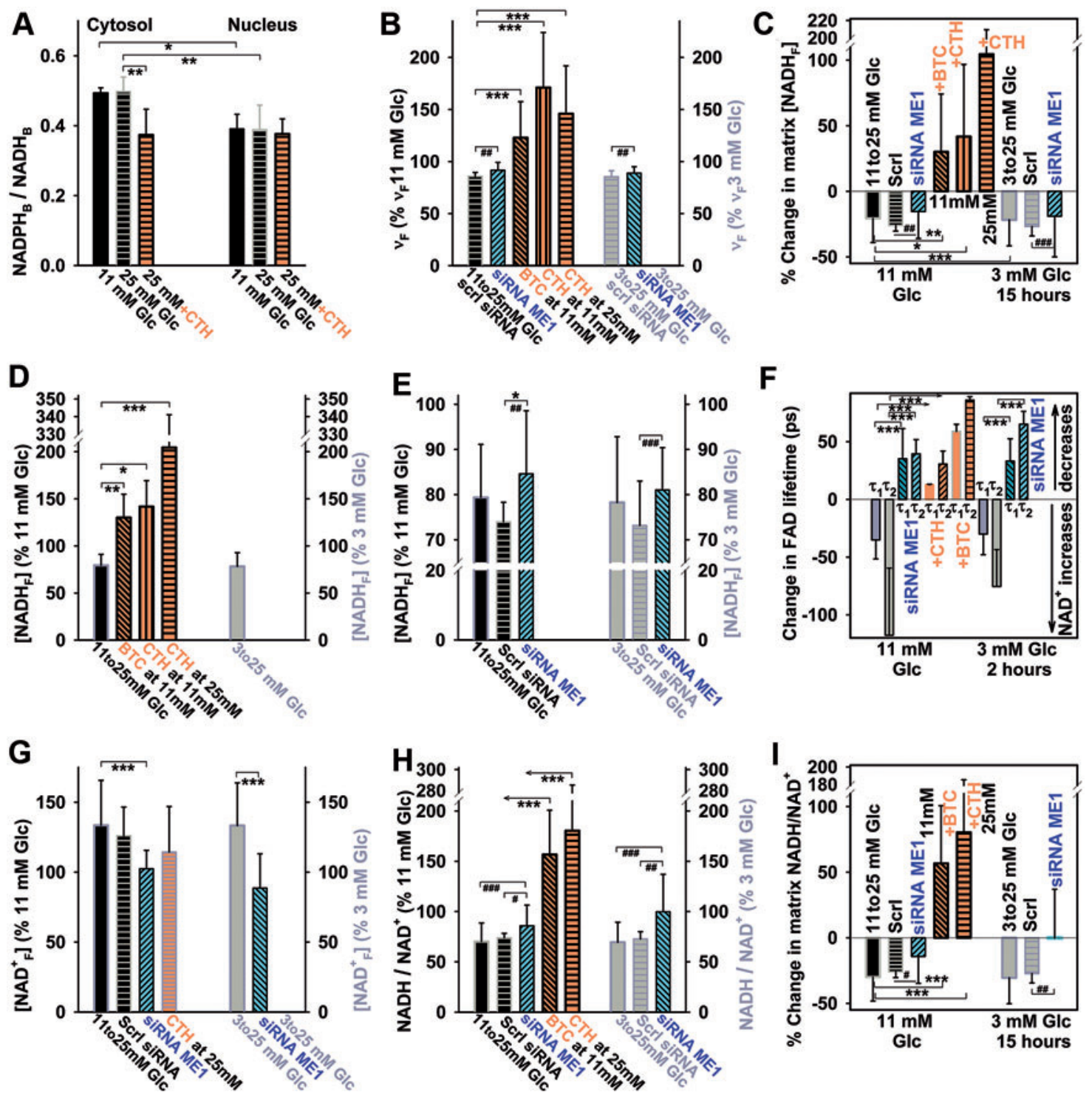

FIG. 8. 2chFLIM-derived changes in mitochondrial matrix NADH, NAD ${ }^{+}$, and NADH/NAD ${ }^{+}$ratio compared with cytosolic bound NADPH/NADH ratios on GSIS. (A) Cytosolic and nuclear-bound NADPH/NADH ratios derived from 2 chFLIM according to Blacker et al. (6) in extramitochondrial ROI and nuclear ROI is shown for INS-1E cells. (B-I) Relative changes in mitochondrial matrix on glucose elevation to $25 \mathrm{~m} M$ for coefficient $v_{\mathrm{F}}$ as calculated by using eq. $\{11\}$ $(\mathbf{B})$; estimated free NADH (unbound; $\left.\mathrm{NADH}_{\mathrm{F}}\right)(\mathbf{C}-\mathbf{E})$; free $\mathrm{NAD}^{+}$(derived from 2chFLIM on the basis of FAD signal quenching by $\mathrm{NAD}^{+}$, using eq. $\left.\{13\}\right)$; and approximated changes in substrate pressure $S$-where $S=\mathrm{NADH}_{\mathrm{F}} / \mathrm{NAD}_{\mathrm{F}}^{+}$and changes are expressed as $S(2) / S(1)$ in percentages, where $S(l)$ denotes the substrate pressure before and $S(2)$ after GSIS for free compounds, using eq. $\{15\}$. BTC, $10 \mathrm{~m} M, \mathrm{CTH}, 0.5 \mathrm{mM}$. Data were calculated by using the integral parameters from the mitochondrial network ROI (except for $\mathbf{A}$ ) and expressed as averages \pm SD of analyzed $N$ biological replicates (2chFLIM $\mathrm{NAD}(\mathrm{P}) \mathrm{H}$ autofluorescence images), each typically containing 80-100 cells, while having $n$ estimations in each. ANOVA: ${ }^{* * *} p<0.001 ; *^{*} p<0.05 ;{ }^{*} p<0.1$; Student's $t$-test: ${ }^{\# \#} p<0.001 ;{ }^{*} p<0.01 ;{ }^{\#} p<0.1$. $N / n$ for INS-1E cells preincubated with $11 \mathrm{~m} M$ glucose was $13 / 31$ (7/17 siRNA ME1; 7/21 with CTH; 3/9 with BTC); whereas with $3 \mathrm{~m} M$ glucose $N / n$ was $16 / 41$ (6/ 16 siRNA ME1). CTH, 4-chloro-3-[[(3-nitrophenyl) amino] sulfonyl]-benzoic acid. Color images are available online.

nucleotides in the mitochondrial matrix and cytosol, we obtained estimates of $\mathrm{NADH} / \mathrm{NAD}^{+}$ratios in the matrix as decreasing on GSIS (Fig. 8H, I). Both ways, division of the estimated changes in $[\mathrm{NADH}]_{\mathrm{F}}$ by those in $\left[\mathrm{NAD}^{+}\right]_{\mathrm{F}}$ and the approximation using eq. $\{15\}$ gave similar results. On CitC inhibition with BTC or CTH and silencing of ME1, the mitochondrial matrix NADH/NAD ${ }^{+}$ratios either remained unchanged or increased (Fig. 8H, I).

\section{Origin of mitochondrial matrix superoxide release}

To ascribe the fractions of mitochondrial matrix superoxide release in INS-1E cells to specific sites of superoxide formation, we employed suppressors of electron leak (Fig. 9A-D), developed by Brand et al. (9). S1QEL exhibited virtually no antioxidant effect at $3 \mathrm{~m} M$ glucose and acted as a pro-oxidant at 11 and $25 \mathrm{~m} M$ glucose (Fig. 9A). At the same 

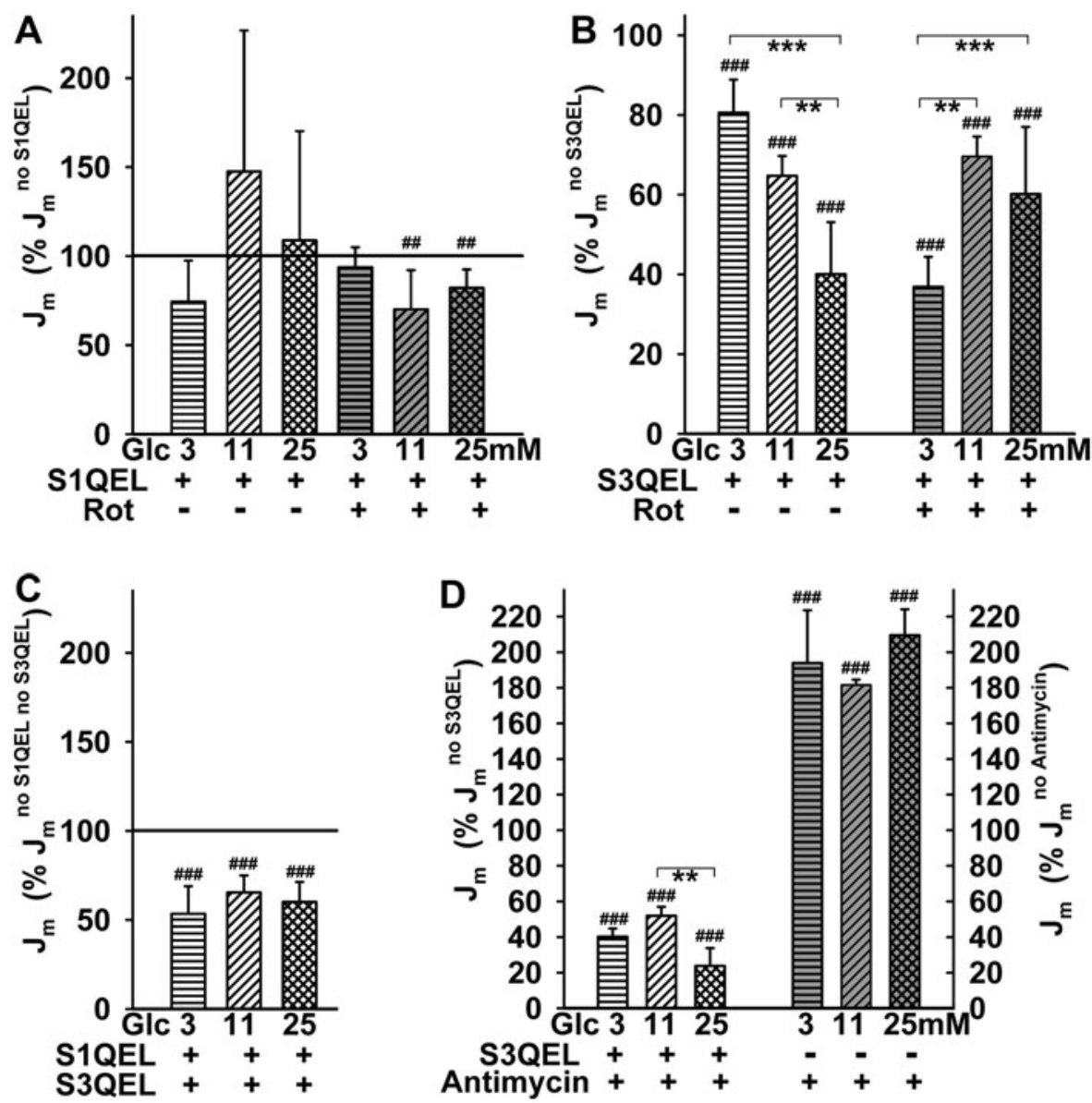

FIG. 9. Effects of suppressors of electron leak at specific sites. (A) Effect of S1QELMitochondrial matrix superoxide release $J_{\mathrm{m}}$ rates were normalized to those without S1QEL at varying glucose concentrations in the absence and presence of rotenone as indicated. (B) Antioxidant capacity of $\mathrm{S} 3 \mathrm{QEL}-J_{\mathrm{m}}$ rates were normalized to those without S3QEL in the absence or presence of rotenone. (C) Simultaneous effects of S1QEL plus S3QEL at $11 \mathrm{~m} M$ glucose. $J_{\mathrm{m}}$ rates were normalized to those without both agents. (D) Antioxidant effect of S3QEL towards Antimycin-induced superoxide production. S1QEL and S3QEL, $10 \mu M$; rotenone, $20 \mu M$. ANOVA

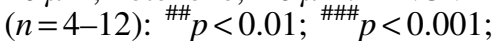
when normalized to $100 \%$. $* * p<0.01 ; \quad * * * p<0.001$; when compared among samples. S3QEL, suppressor of complex 3 site Q electron leak.

time, S1QEL exerted a weak antioxidant effect against rotenone-induced superoxide production at 11 and $25 \mathrm{mM}$ glucose (Fig. 9A) (29). These results demonstrate that both rotenone and $\mathrm{S} 1 \mathrm{QEL}$ interfere within the $\mathrm{I}_{\mathrm{Q}}$ site of Complex I in the vicinity of the ubiquinone binding site (29).

In contrast, the suppressor of Complex III site $\mathrm{Q}$ electron leak (S3QEL) exhibited an antioxidant effect that increased with increasing glucose, diminishing $J_{\mathrm{m}}$ rates down to $81 \%$, $65 \%$, and $40 \%$ at 3,11 , and $25 \mathrm{mM}$ glucose, respectively (Fig. 9B). Indirectly, these results indicate that the remaining portion of the superoxide matrix release, which also includes the contribution from the $\mathrm{I}_{\mathrm{F}}$ site, decreases with increasing glucose on GSIS. This perfectly correlates with the decreasing substrate pressure $S$. Thus, on GSIS with maximum glucose, at least $60 \%$ of the superoxide released into the mitochondrial matrix originates from the Complex III outer $\mathrm{Q}$ site $\mathrm{III}_{\mathrm{Qo}}(7,8,29)$. In contrast, at $3 \mathrm{~m} M$ glucose, the contribution of site $\mathrm{III}_{\mathrm{Qo}}$ is only $20 \%$ and the remaining superoxide is produced predominantly from the $\mathrm{I}_{\mathrm{F}}$ site due to the high substrate pressure.

Unexpectedly, S3QEL also exhibited an antioxidant effect against rotenone, being the most efficient at $3 \mathrm{mM}$ glucose (Fig. 9B). The site specificity for Complex III was confirmed by the observation of the nearly complete S3QEL-mediated suppression of superoxide release induced by antimycin A (Fig. 9D). Further, addition of S1QEL together with S3QEL did not further decrease the $J_{\mathrm{m}}$ rates, which remained at $53 \%, 65 \%$, and $60 \%$ at 3,11 , and $25 \mathrm{mM}$ glucose, respec- tively (Fig. 9C). Comparing this with the effects of S3QEL alone, the slight pro-oxidant action of S1QEL still contributes to the composite effect.

\section{Discussion}

We described a decline in mitochondrial superoxide formation during glucose-induced insulin secretion in rat pancreatic $\beta$ cells (INS-1E cells) and PIs. We not only confirmed the recently reported glucose-induced reduction in oxidation of mitochondrial matrix-targeted roGFP2-Orp1 (15) but also elucidated the mechanism for the decreased pro-oxidant state, established in the mitochondrial matrix on GSIS. A concomitant drop in the matrix $\mathrm{NADH}_{\mathrm{F}} / \mathrm{NAD}_{\mathrm{F}}^{+}$and $\mathrm{NADPH}_{\mathrm{F}} / \mathrm{NADP}_{\mathrm{F}}^{+}$was indicated, in contrast to elevations in the cytosolic $\mathrm{NADPH}_{\mathrm{F}}$ (Fig. 10A). Since the decreased matrix $\mathrm{NADPH}_{\mathrm{F}} / \mathrm{NADP}^{+}{ }_{\mathrm{F}}$ ratio may cause equivalent exhaustion of matrix reduced glutathione (GSH), we may conclude that the acute pro-oxidant state decrease in mitochondria on GSIS is established at the expense of susceptibility to the oxidative stress.

The acute phenomenon results from the finely tuned bioenergetics of the OXPHOS metabolism of glucose, switching toward increasing respiratory chain substrates in pancreatic $\beta$ cell mitochondria and increasing the activity of three mitochondrial redox shuttles that subsequently export reducing equivalents to the cytosol (Fig. 10B, C). The resulting elevated cytosolic $\mathrm{NADPH}_{\mathrm{F}}$ facilitates insulin secretion (34). 
A

FIG. 10. Major metabolic fluxes and redox shuttles contributing to diminished mitochondrial superoxide generation in pancreatic $\beta$ cells on GSIS. (A) Overview of changes on GSIS in the cytosol and mitochondrial matrix; (B) Cells at low glucose; (C) cells on glucose intake: Higher rates of metabolic fluxes are depicted by thick arrows, whereas lower rates are shown by dotted arrows and decreasing products are depicted in gray fonts or symbols. The color coding for shuttles and abbreviations are the same as in Figure 1. Similarly, higher superoxide formation rate is indicated with thick arrows, whereas slow rate is depicted with thin arrows. Color images are available online.
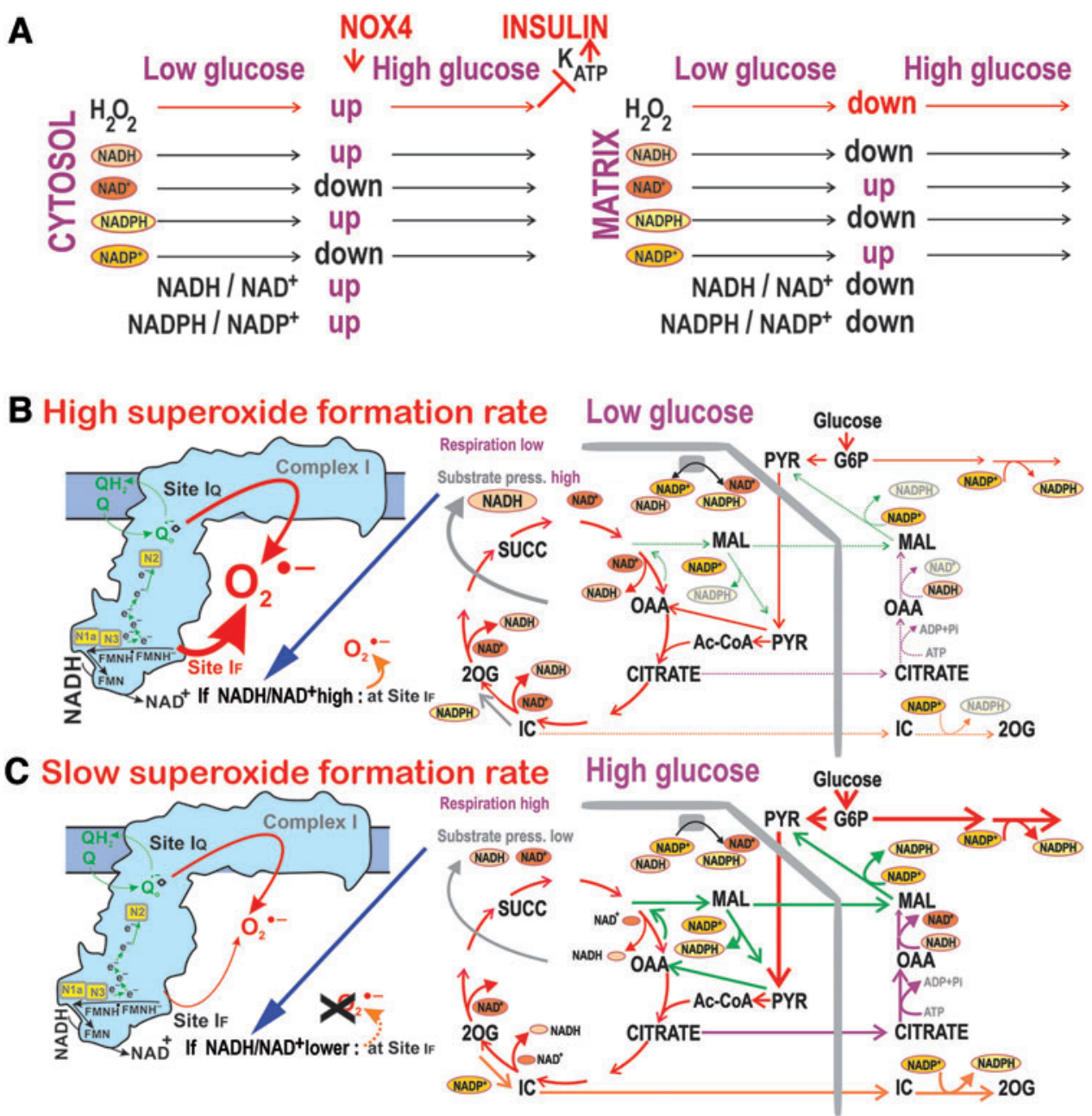

Recently, we explained how, when revealing the existence of the essential NOX4-mediated cytosolic redox signaling, which together with ATP elevations fundamentally determines insulin exocytosis (48). A portion of $\mathrm{NADPH}_{\mathrm{F}}$ required for NOX4 reaction comes from the redox shuttles described here. Other substantial $\mathrm{NADPH}_{\mathrm{F}}$ supply for NOX4 is produced by two of the PPP enzymes on GSIS.

Since $\beta$ cells lack a significant lactate dehydrogenase activity and functional pyruvate dehydrogenase kinases, nearly $100 \%$ of pyruvate originating from glycolysis is utilized by the Krebs cycle $(2,10,52,55)$ to sustain OXPHOS (Figs. 1A, $\mathrm{B}$ and 10B, C). Pyruvate is about equally metabolized by PDH and pyruvate carboxylase (52). The PDH enables an increase in the Krebs cycle rate, followed by the increased respiration (60) and OXPHOS, thus increasing ATP, which subsequently contributes to $\mathrm{K}_{\mathrm{ATP}}$ closing and GSIS.

If only PDH was functional and without operating redox shuttles, the resulting incremental increase in NADH and concomitant elevation of respiration would cause a higher extent of proton pumping and establish a high protonmotive force $\Delta p$ (including higher $\Delta \Psi_{\mathrm{m}}$ ). The concomitantly increased ATP synthesis would transport more protons back through the ATP-synthase. The resulting $\Delta p$ would thus be established as somewhat smaller than without this proton backflow. As a result, also mitochondrial superoxide formation would decrease (Hypothesis II). This com- ponent is a basic one, fundamentally contributing to the mitochondrial reductive state.

However, with the additional pyruvate carboxylase reaction and concomitant malate efflux from the matrix, enabled by $2 \mathrm{OGC}$, the generated OAA can either increase the turnover of the Krebs cycle or be converted by the reverse MDH reaction and thus initiate the pyruvate/malate shuttle (Figs. 1A and 10B, C). MDH then produces less NADH than would be produced without the redox shuttle. Its operation was proven here by the effects of ME1 and pyruvate carboxylase silencing and inhibitors of the respective carriers.

Crucially, this lower matrix NADH availability is also given by the lower IDH3-mediated NADH formation due to the effective citrate and isocitrate efflux within the pyruvate/ citrate and pyruvate/isocitrate shuttles, respectively. Their operation provides much lower matrix NADH/NAD ${ }^{+}$ratios than in their absence. Matrix NADH in $\mu M$ but $\mathrm{NAD}^{+}$of $>500 \mu M$ seems to be typical (12). Indeed, functionality of the pyruvate/isocitrate shuttle was independently supported by ${ }^{13} \mathrm{C}$-incorporation experiments (Fig. 6C), unequivocally determining the counter-Krebs cycle direction of isocitrate flux (59) and its increase at high glucose.

Under the hypothetical maximum substrate elevation without redox shuttles, one would have anticipated elevated superoxide formation due to the expected higher matrix $\mathrm{NADH} / \mathrm{NAD}^{+}$ratios. Such a high matrix substrate pressure $S$ 
$\left(\mathrm{NADH}_{\mathrm{F}} / \mathrm{NAD}_{\mathrm{F}}^{+}\right)$would cause higher superoxide formation at site $\mathrm{I}_{\mathrm{F}}$ in the vicinity of the flavin binding site of Complex I $(8,50)$. The only situations that followed the most simple expectation of an increased superoxide formation with increased substrates occurred (i) in media completely depleted of glucose and pyruvate-free on glucose addition (Fig. 3A) or (ii) in cells with an inhibited citrate or malate export; or with two redox shuttles blocked by ME1 silencing, partly with pyruvate/isocitrate shuttle blocked by IDH2 silencing, or completely with simultaneous silencing of pyruvate carboxylase and inhibited citrate export; that is, again with inhibited redox shuttles. Otherwise, with initial 3 and $11 \mathrm{~m} M$ glucose, the sudden glucose intake slows down superoxide formation released to the mitochondrial matrix (Fig. 3A-E).

The three active redox shuttles allow elevations of cytosolic $\mathrm{NADPH}_{\mathrm{F}}$ on GSIS $(34,35)$ (Figs. 5B-D and 8A) at the expense of the diminished mitochondrial matrix $\mathrm{NADH}_{\mathrm{F}}$ and a concomitant $\mathrm{NAD}_{\mathrm{F}}^{+}$increase. Its existence was also supported by a 3.2-fold increase in $\beta$-OHB (Fig. 6C and Supplementary Fig. S2Fa). Consequently, the resulting matrix substrate pressure $\left(\mathrm{NADH}_{\mathrm{F}} / \mathrm{NAD}_{\mathrm{F}}^{+}\right)$is also diminished (Fig. 8H, I). When these shuttles are shut down by combinations of the metabolite carrier inhibitors with silencing of ME1, IDH2, or pyruvate carboxylase, the substrate pressure $\mathrm{NADH}_{\mathrm{F}} / \mathrm{NAD}_{\mathrm{F}}^{+}$does not decrease but instead increases (Fig. 8H, I). Thus, an elevated matrix superoxide release stems from the inhibited redox shuttles (Fig. 3A-D). Concomitantly, elevation of the cytosolic $\mathrm{NADPH}_{\mathrm{F}}$ at high glucose vanishes with the blocked carriers and/or shuttles (Figs. 5A-D and 8A and Supplementary Fig. S7). The maintenance of $\mathrm{NAD}^{+}$may also maintain the ongoing Sirtuin-3mediated de-acetylation of proteins and factors that are crucial for OXPHOS $(41,63)$. Moreover, the cataplerotic flux, ensured by the redox shuttles, acts upstream of NNT; hence, the phenomenon is preserved independently of the NNT deficiency.

We have excluded the predominant participation of other mechanisms, hypothetically decreasing superoxide formation on GSIS (Supplementary Part II; Supplementary Fig. S2). A third one could theoretically be a switch from fatty acid to glucose metabolism (24), since fatty acid $\beta$-oxidation produces additional ROS by electron transfer flavoprotein:quinone oxidoreductase. However, since the decreasing ROS were also observed with etomoxir, an inhibitor of $\beta$-oxidation, this mechanism should not significantly contribute. The fourth mechanism would be based on the well-known elevation of cytosolic $\mathrm{Ca}^{2+}$ on GSIS that could be relayed by the increased $\mathrm{Ca}^{2+}$ uniport and compensating $\mathrm{Ca}^{2+} / 2 \mathrm{Na}^{+}$antiport ensuring the $\mathrm{Ca}^{2+}$ efflux (Supplementary Fig. S2Bc). Since we observed the decrease in $J_{\mathrm{m}}$ rates with blocked $\mathrm{Ca}^{2+}$ uniporter, either the three redox shuttles are still operating or variations in mitochondrial $\mathrm{Ca}^{2+}$ fluxes on GSIS probably do also contribute to these declines. We may still anticipate the participation of changed $\mathrm{Ca}^{2+}$ fluxes on GSIS.

The fifth possible mechanism might originate from the functional malate/aspartate shuttle decreasing respiration at low glucose versus its slow-down at high glucose. However, without external glutamine or on silencing of the aspartate/ glutamate carriers, the decrease in the mitochondrial matrix superoxide release and accumulated superoxide $/ \mathrm{H}_{2} \mathrm{O}_{2}$ was maintained after glucose intake. Moreover, the malate/ aspartate shuttle (Fig. 1B) cannot proceed simultaneously with the three redox shuttles of Figure 1A. Nevertheless, its elimination under the low-glucose conditions (insulin nonstimulating) led to higher oxidative conditions, indicating a higher superoxide formation at retarded metabolism.

The finding of decreased mitochondrial superoxide formation on GSIS is itself a remarkable fact. This is a great phylogenetic discovery that mitochondria, representing the ATP branch of the glucose sensor in pancreatic $\beta$ cells, do not acutely contribute to oxidative stress while the sensor is functioning. Perhaps this allows $\beta$ cells to afford a much lower cytosolic antioxidant buffer capacity than the other cell types (30). However, since matrix $\mathrm{NADPH}_{\mathrm{F}}$ decreases on GSIS, which might decrease matrix GSH, these repetitive transient decreases might contribute to oxidative stress. One can consider $\beta$ cells as perfect redox machines, since they are rich in disulfide reductase-based antioxidant defenses (27). This, together with a lowered antioxidant buffer capacity allows fine redox signaling (50), on insulin secretion stimulated with glucose and branched-chain ketoacids (48). We should pay attention to these intermittent declines in the matrix antioxidant capacity, similar to the accumulated oxidative stress amplified by lipotoxicity, and glucotoxicity, which are major factors in the development of type 2 diabetes [reviewed in Aon et al. (1), Ivarsson et al. (27), Ježek et al. (28), Lombard and Zwaans (41), and Plecitá-Hlavatá et al. (49)].

In the vicinity of the Complex I flavin $\mathrm{I}_{\mathrm{F}}$ site, superoxide formation increases at higher $\mathrm{NADH} / \mathrm{NAD}^{+}$; otherwise, superoxide formation decreases $(40,65)$. Interestingly, while probing superoxide formation sites with suppressors of electron leak at the specific Complex I or Complex III ubiquinone-binding sites (9), the S3QEL suppressor diminished superoxide release into the mitochondrial matrix more intensively with increased glucose (down to $\sim 40 \%$ at $25 \mathrm{mM}$ glucose). Thus with maximum glucose, substantial superoxide formation (up to $60 \%$ of the matrix-released superoxide) takes place within the outer site $\mathrm{III}_{\mathrm{Qo}}$. At this site, superoxide is also formed in the presence of antimycin A. This reflects the existence of fast electron flow at elevated respiration, which is, however, not matched by the sufficient capacity of cytochrome $c$ shuttling. As a result, the electron flow is retarded at the $\mathrm{III}_{\mathrm{Qo}}$ site and allows superoxide formation. Since there is an increasing fraction of superoxide ascribed to the $\mathrm{III}_{\mathrm{Qo}}$ site with the increasing glucose, this means that the remaining fraction, where the Complex $\mathrm{I}_{\mathrm{F}}$ site contributes, is higher at lower glucose and therefore at higher $\mathrm{NADH}_{\mathrm{F}} /$ $\mathrm{NAD}_{\mathrm{F}}^{+}$. In contrast, the Complex $\mathrm{I} \mathrm{I}_{\mathrm{F}}$ site contribution is lower at high glucose. Consequently, only the fraction of matrix superoxide release formed at the $\mathrm{I}_{\mathrm{F}}$ site decreases with the acute increase in glucose in $\beta$ cells. The fraction given by the $\mathrm{III}_{\mathrm{Qo}}$ site then logically increases.

\section{Materials and Methods}

\section{Materials}

Reagents, including CitC inhibitors BTC and 4-Chloro-3[[(3-nitrophenyl) amino] sulfonyl]-benzoic acid (CTH), were from Sigma Aldrich (St. Louis, MO), unless stated otherwise. S1QEL and S3QEL were from Life Chemicals (shipped from Spoluka Chemical Company, Kiev, Ukraine). 


\section{Cell and PI cultures}

Rat insulinoma INS-1E cells (kindly provided by Prof. Maechler, University of Geneva or purchased from AddexBio, San Diego, CA; cat. No. C0018009) were cultured in RPMI 1640 medium supplemented with $11 \mathrm{~m} M$ glucose, $5 \%$ (v/v) fetal calf serum, $10 \mathrm{~m} M$ HEPES, $1 \mathrm{~m} M$ pyruvate, $50 \mu M$ mercaptoethanol, $50 \mathrm{IU} / \mathrm{mL}$ penicillin, and $50 \mu \mathrm{g} / \mathrm{mL}$ streptomycin (55). The 1-, 2-, or 15-h incubations were performed in RPMI 1640 with $3 \mathrm{~m} M$ glucose to lower glucose and reduced beneficial autocrine effects (51). CitC, ME1, pyruvate carboxylase, SLC25A12/AGC1/aralar, SLC25A13/AGC2, NNT, and IDH2 silencing were performed by transfections, facilitated by RNAiMax (Thermo Fisher Scientific, Waltham, MA), with properly predesigned siRNAs (Sigma), that is, for CitC (SASI_Rn01_00120045 and SASI_Rn01_ 00120046); ME1 (NM_012600, SASI_Rn02_00259753 and NM_012600,SASI_Rn02_00259754); pyruvate carboxylase (NM_012744, SASI_Rn01_00101243); SLC25A12/AGC1/ aralar (XM_342445, SASI_Rn02_00394279 and SASI_ Rn02_00394280); and SLC25A13/AGC2 (XM_001054092, SASI_Rn02_00274696 and SASI_Rn02_00274697), likewise SASI_Rn01_00066518 and SASI_Rn01_00066519 for NNT and SASI_Rn01_00093144 and SASI_Rn01_ 00093145 for IDH2. PIs were isolated from C57BL/6 J mice (or when indicated from C57BL/6N mice) and used immediately for measurements. Nevertheless, they could be maintained for up to 1 week in the transient culture as previously described $(17,19,60)$.

\section{Confocal microscopy assay of surplus superoxide matrix release}

A Leica TCS SP2 AOBS, or alternatively Leica TCS SP8, was employed for the MitoSOX Red (Thermo Fisher) monitoring of in situ superoxide surplus release to the mitochondrial matrix by using $514 \mathrm{~nm}$ excitation and $610-679 \mathrm{~nm}$ emission. Rates, that is, integral fluorescence intensity increases $\left(J_{\mathrm{m}}\right)$ with time, were derived in the region of interest, which was the mitochondrial network. Note that this method is feasible for the semi-quantification of mitochondrial superoxide release rates even at low or collapsed $\Delta \Psi_{\mathrm{m}}$, since MitoSOX Red permanently intercalates into mitochondrial DNA (mtDNA) and cannot leak out (see also the Supplementary Data) (18).

However, even when surveying the rates, this method is frequently criticized and it is believed that it is unable to account for matrix superoxide changes $(36,67,68)$. Indeed, if MitoSOX Red molecules were freely membrane permeable and not bound to mtDNA, fluorescence signal changes would potentially originate from the changes of either inner mitochondrial membrane (IMM) potential $\Delta \Psi_{\mathrm{m}}$ or plasma membrane potential $\Delta \Psi_{\mathrm{p}}$. However, as shown in the Supplementary Data, three aspects support the independence of the two potentials $\Delta \Psi_{\mathrm{m}}$ and $\Delta \Psi_{\mathrm{p}}$ under certain conditions that are suitable for feasible MitoSOX Red-based confocal microscopy assays. This is valid even for pancreatic $\beta$ cells, where both potentials definitely change on the addition of glucose to cells preincubated in a medium containing lower glucose concentration. On glucose stimulation of $\beta$ cells, the IMM potential $\Delta \Psi_{\mathrm{m}}$ slightly increases, whereas the blockage of $\mathrm{K}_{\mathrm{ATP}}$ stops the hyperpolarizing current at the plasma membrane and the subsequent burst of action potential depolarizes $\Delta \Psi_{\mathrm{p}}$ to at least zero $(2,52,55)$.

After their addition to cells, free membrane-permeant hydrophobic MitoSOX Red cationic molecules would redistribute between medium and cell cytosol according to the plasma membrane potential $\Delta \Psi_{\mathrm{p}}$. In addition, MitoSOX Red cations would redistribute between the mitochondrial matrix and cytosol according to $\Delta \Psi_{\mathrm{m}}$. Since $\Delta \Psi_{\mathrm{m}}$ usually amounts to $\sim 180 \mathrm{mV}$ and $\Delta \Psi_{p}>60 \mathrm{mV}$, one might expect four orders of magnitude MitoSOX Red accumulation in the mitochondrial matrix space relative to the medium. After the addition of glucose, such an accumulation and hence fluorescence signal may decrease at least 10 -fold due to plasma membrane depolarization, if the other aspects are not considered.

However, due to the first aspect being considered, that is, that in $\beta$ cells $\Delta \Psi_{\mathrm{m}}$ increases after the glucose addition (60), it would be reasonable to expect a $\Delta \Psi_{\mathrm{m}}$ increase of no more than $10 \mathrm{mV}$. Hence, MitoSOX Red cation accumulation will be additionally $\sim 1.5$ times higher in the mitochondrial matrix after glucose addition. This must lead to a much smaller decrease in the fluorescence signal, even for the freely penetrating cation.

The second aspect stems from the absence of superresolution for this confocal microscopy assay. Conventional confocal microscopy does not afford a better resolution than $200 \mathrm{~nm}$, neither $20 \mathrm{~nm}$, which would be required to resolve cristae, because mitochondrial ROI (sections of mitochondrial network tubules) in conventional confocal images represent a mixture of signals originating from the intracristal space (freely accessible for small compounds from the cytosol) and the mitochondrial matrix space (due to the "zebra" of cristae resulting from mitochondrial network tubule optical sections) $(50,51)$. For cells with rich cristae, such as pancreatic $\beta$ cells, the mitochondrial matrix signal readout would only account for around half of the fluorescence signal, whereas the remaining half would comprise, in fact, the cytosolic concentration of the MitoSOX Red cation. This contributes to the fluorescence background. Consequently, when the rates are not taken into the account and only the fluorescence intensity is considered, quantification is obscured.

The third aspect lies in the ability of MitoSOX Red to intercalate into mtDNA (18). The intercalated pool of MitoSOX Red typically dominates the confocal microscopy signal (18). Consequently, on certain values of intercalation fraction (see the calculation in the Supplementary Data, Supplementary Table S1), the integral fluorescence intensity within the mitochondrial $\mathrm{ROI}$ is almost insensitive to $\Delta \Psi_{\mathrm{m}}$ $(28,29)$. Despite these theoretical considerations, the experimental sensitivity of MitoSOX Red fluorescence toward $\Delta \Psi_{\mathrm{m}}$ changes may be even lower than predicted.

The mtDNA-intercalated MitoSOX Red pool is also completely insulated from accessing the cytosol or mitochondrial membranes, since it does not respond to very high doses of membrane-permeant hydrophobic pro-oxidants such as tert-butyl hydroperoxide (28). The disadvantage of this approach lies in a possible toxicity that could prevent correct long-term cell responses during their further culturing. Nevertheless, $20 \mathrm{~min}$ time-lapsed confocal microscopy recordings were found to not affect cells. However, repeated sampling with new coverslips and cell samples is recommended for long time intervals. In this way, snapshots of superoxide release rates are obtained, which provide insights 
into important physiological phenomena such as the redox initiation of the hypoxia-inducible factor signaling, that is, the peak in $J_{\mathrm{m}}$ rates occurring after $5 \mathrm{~h}$ of hypoxic incubation (Plecitá et al., unpublished observations).

\section{Confocal microscopy assay of $\mathrm{H}_{2} \mathrm{O}_{2}$ release into the mitochondrial matrix within intact cells}

The HyPer family of fluorescence probes has been developed for the selective detection of $\mathrm{H}_{2} \mathrm{O}_{2}(3,4,38,54)$. We have employed vectors encoding mito-HyPer and mitoSypHer (kindly provided by Dr. Enyedi, Semmelweis University, Hungary). INS-1E cells were transfected with either one of these vectors, with the help of Lipofectamine 2000 (Thermo Fisher Scientific) for $24 \mathrm{~h}$ before measurements. A Leica TCS SP8 confocal microscope was employed for the intermittent recording of integral fluorescence intensity $(F)$ in the mitochondrial network ROI with excitation at 405 and $488 \mathrm{~nm}$, respectively.

To completely eliminate any potential $\mathrm{pH}$ dependency of mito-HyPer, the entire signal (in fact the fluorescence intensity per unit or chosen constant area of mitochondrial ROI) of the $\mathrm{H}_{2} \mathrm{O}_{2}$-insensitive but $\mathrm{pH}$-sensitive probe mitoSypHer was subtracted from the mito-HyPer fluorescence signal for both excitations at 488 and $405 \mathrm{~nm}$ :

$$
\Delta F_{488}^{H}=F_{488}^{H}-F_{488}^{S} \text { and } \Delta F_{405}^{H}=F_{405}^{H}-F_{405}^{S}
$$

The resulting differential fluorescence data $(\Delta F)$ were used to calculate the ratios $R\left(\mathrm{H}_{2} \mathrm{O}_{2}\right)$ of corrected mito-HyPer emission: $R\left(\mathrm{H}_{2} \mathrm{O}_{2}\right)=\Delta F_{485}{ }_{4} / \Delta F_{405}{ }_{40}$. The relative $J_{\mathrm{m}}{ }^{\mathrm{H} 2 \mathrm{O} 2}$

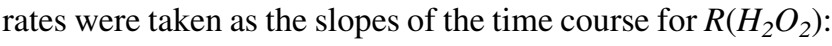

$$
J_{m}{ }^{\mathrm{H} 2 \mathrm{O} 2}=\frac{\mathrm{d}}{\mathrm{dt}} F_{488}^{H}-F_{488}^{S} / F_{405}^{H}-F_{405}^{S}
$$

The typical resulting traces for $\left.\mathrm{R}_{(} \mathrm{H}_{2} \mathrm{O}_{2}\right)$ versus time are shown in Figure 3I-K. $R\left(\mathrm{H}_{2} \mathrm{O}_{2}\right)$ increased significantly on the addition of a $200 \mu M \mathrm{H}_{2} \mathrm{O}_{2}$ aliquot, confirming the correct mito-HyPer response $(15,54)$. A $J_{\mathrm{m}}{ }^{\mathrm{H} 2 \mathrm{O} 2}$ elevation was also detected with the addition of rotenone (Fig. 3K, L) or antimycin A (see Supplementary Data) and decreased after the subsequent addition of the mitochondrial matrix antioxidants SkQ1 or S1QEL. The slopes of the derived dependencies $J_{\mathrm{m}}{ }^{\mathrm{H} 2 \mathrm{O} 2}$ were taken as upper limits of the observed rates and were converted to approximate values in nmols $\cdot \mathrm{s}^{-1}$ by the known extent of changes after the addition of $\mathrm{H}_{2} \mathrm{O}_{2}$ aliquots. Such calibration is approximate due to the fact that not all added external $\mathrm{H}_{2} \mathrm{O}_{2}$ penetrates into the mitochondrial matrix and influences the probes localized there.

\section{MitoB LC-MS assay of mitochondrial ROS}

The mitochondrial matrix-targeted $\mathrm{H}_{2} \mathrm{O}_{2}$-specific probe MitoB was used to quantify accumulated ROS in the mitochondrial matrix over time, using an adopted method (14). The boron-containing MitoB is oxidized in the mitochondrial matrix to MitoP, and both species are quantified by LC-MS. MitoP/MitoB ratios are then taken as proportional to $\mathrm{H}_{2} \mathrm{O}_{2}$ (ROS) accumulated within the timeframe of the experiment ( $2 \mathrm{~h}$ in our case).

The INS-1E cells were grown under the standard conditions described earlier and preincubated in medium con- taining 3 or $11 \mathrm{~m} M$ glucose, respectively, for $2 \mathrm{~h}$ at $37^{\circ} \mathrm{C}$. Next, the medium was replaced with a fresh one but supplemented with $5 \mu M$ MitoB probe (Sigma Aldrich). When indicated, glucose was increased to $25 \mathrm{mM}$. Cells were then incubated for $2 \mathrm{~h}$ at $37^{\circ} \mathrm{C}$. In separate runs, $20 \mu M$ mitoparaquat (Abcam) was included, which generates $\mathrm{H}_{2} \mathrm{O}_{2}$ within the mitochondrial matrix. Thus, we obtained a positive control.

After the treatment, $500 \mu \mathrm{L}$ aliquots were removed from the reaction, snap-frozen on dry ice, and stored at $-80^{\circ} \mathrm{C}$ before further processing. For MitoB and MitoP quantification, samples were thawed and $200 \mu \mathrm{L}$ aliquots were transferred to new tubes. All samples were spiked with $500 \mathrm{n} M$ internal standards of $\mathrm{d}_{15}$-MitoB and $\mathrm{d}_{15}$-MitoP (Cayman Chemicals) and vortexed for $30 \mathrm{~s}$. Fifty microliters of $100 \%$ acetonitrile/ $0.1 \%$ formic acid (vol/vol) was added; the samples were vortexed again for $30 \mathrm{~s}$, and they were centrifuged for $10 \mathrm{~min}$ at $16,000 \mathrm{~g}$ at room temperature. Subsequently, $100 \mu \mathrm{L}$ sample aliquots were used for LC-MS analysis.

Mass spectra were obtained by using a Shimadzu Prominence system consisting of a DGU-20A3 mobile phase degasser, two LC-20AD solvent delivery units, an SIL-20AC cooling autosampler, a CTO-10AS column oven, SPD-M20A diode array, and LCMS-2020 mass detectors with a single quadrupole equipped with an electrospray ion source (Shimadzu, Kyoto, Japan). Binary gradient elution was used as follows: mobile phase $\mathrm{A}=$ water, $0.1 \%$ formic acid; mobile phase $\mathrm{B}=100 \%$ acetonitrile; linear gradient: 0 min $30 \%$ $\mathrm{B}, 6 \mathrm{~min} 60 \% \mathrm{~B}$; and $7 \mathrm{~min} 30 \% \mathrm{~B}, 10 \mathrm{~min}$ stop. The flow rate was $0.4 \mathrm{~mL} \cdot \mathrm{min}^{-1}$ at $25^{\circ} \mathrm{C}$, and the injection volume was $10 \mu \mathrm{L}$.

The MS parameters were as follows: Positive mode was used, whereas the ESI interface voltage was $4.5 \mathrm{kV}$; detector voltage was $1.15 \mathrm{kV}$, the nebulizer gas flow was $1.5 \mathrm{~mL} \cdot \mathrm{min}^{-1}$, drying gas flow was $15 \mathrm{~mL} \cdot \mathrm{min}^{-1}$, heat block temperature was $200^{\circ} \mathrm{C}$, DL temperature was $250^{\circ} \mathrm{C}$, and the SIM mode was 397 for Mito $\mathrm{B}[\mathrm{M}+\mathrm{H}]^{+}, 369$ for Mito P $[\mathrm{M}+\mathrm{H}]^{+}, 412$ for Mito B deut. $[\mathrm{M}+\mathrm{H}]^{+}$, and 384 for Mito $\mathrm{P}$ deut. $[\mathrm{M}+\mathrm{H}]^{+}$. The software LabSolutions version 5.75 SP2 was used for data quantification. The ratio of MitoP/MitoB was estimated from the respective areas under the curve obtained by the MS analysis.

\section{Enzymatic NADPH assay}

Total cell NADPH was quantified by using a kit (BioVision, Milpitas, CA).

\section{Confocal microscopy assay of cytosolic and matrix NADPH}

The iNAP family of NADPH-selective fluorescence probes discriminating between NADPH and NADH was developed by Dr. Yi Yang (East China University of Science and Technology, Shanghai, China) (64), who kindly provided us with iNAP1 for cytosolic and iNAP3 for matrix monitoring and the nonresponding control probe iNAPc. A Leica TCS SP8 confocal microscope was used for the intermittent recording of integral fluorescence $(F)$ within ROI of each individual cell (up to 15 cells in total) with excitation at 405 and $488 \mathrm{~nm}$. The $F_{405} / F_{488}$ ratios derived from each individual cell were then averaged. Since these ratios are directly proportional to cytosolic or mitochondrial matrix NADPH 
concentration $[\mathrm{NADPH}]_{\mathrm{c}}$ or $[\mathrm{NADPH}]_{\mathrm{m}}$, respectively, the average elevation after the addition of $25 \mathrm{mM}$ glucose $\Delta[\mathrm{NADPH}]_{\mathrm{c}}$ or $\Delta[\mathrm{NADPH}]_{\mathrm{m}}$, respectively, was set as $100 \%$ and elevations in $\Delta[\mathrm{NADPH}]_{\mathrm{c}}$ or $\Delta[\mathrm{NADPH}]_{\mathrm{m}}$ relative with the tested agents were expressed normalized to this average $(100 \%)$ change in their absence.

\section{Double-channel FLIM assay for separation of NADPH and NADH and estimation of $N A D^{+}$}

A Coherent Chameleon Ultra I mode-locked Ti:sapphire laser with 140 fs pulse width was used in conjunction with a Leica TSC SP8 confocal microscope and an attachment for 2chFLIM (Becker \& Hickl, Berlin, Germany). The laser was tunable from 690 to $1040 \mathrm{~nm}$ with $2.9 \mathrm{~W}$ of average power at the peak of the tuning range, which provides $\sim 500 \mathrm{~mW}$ at $980 \mathrm{~nm}$. To assay NAD(P)H by autofluorescence in situ, twophoton confocal excitation was set up as follows: Both $\mathrm{NAD}(\mathrm{P}) \mathrm{H}$ and FAD were excited at $700 \mathrm{~nm}$ whereas NAD(P)H was detected at $467-499 \mathrm{~nm}$ emission and timeresolved fluorescence decay yields were obtained by iterative re-convolution using SPCImage (Becker \& Hickl) as two lifetimes for free and bound molecules $\tau_{\mathrm{F}}=0.4-0.5 \mathrm{~ns}$ and $\tau_{\mathrm{B}}$ $\sim 2.6 \mathrm{~ns}$, respectively (6), and their weight coefficients $\alpha_{\mathrm{F}}$ and $\alpha_{\mathrm{B}}$ (normalized amplitudes for which $\alpha_{\mathrm{F}}=1-\alpha_{\mathrm{B}}$ ), respectively. Average lifetimes integrated over ROI encompassed either the mitochondrial network or extra-mitochondrial compartments or nuclei in each separate coverslip. Only a few cells were taken for calculation. The FAD was detected at $500-550 \mathrm{~nm}$ emission, yielding typically shorter lifetimes for bound molecules $\tau_{\mathrm{B}}^{\mathrm{FAD}}$ and long lifetimes for free $\tau_{\mathrm{F}}^{\mathrm{FAD}}(6,26)$. Fluorescence lifetime $\tau_{\mathrm{FAD}}^{\mathrm{FA}}$ has previously been found to decrease in the presence of $\mathrm{NAD}^{+}$because of fluorescence quenching (26).

The general $\mathrm{NAD}(\mathrm{P}) \mathrm{H}$ fluorescence decay $I(t)$ can be expressed as:

$$
I(t)=Z+I_{0} \cdot\left[\alpha_{\mathrm{F}} \cdot \exp \left(-t / \tau_{\mathrm{F}}\right)+\alpha_{\mathrm{B}} \cdot \exp \left(-t / \tau_{\mathrm{B}}\right)\right],
$$

where the weight coefficients $\alpha_{\mathrm{F}}, \alpha_{\mathrm{B}}$ reflect a mixture of $\mathrm{NADH}$ and NADPH, $t$ is time in ns, and $Z$ and $I_{0}$ are constants. We assumed that roughly the same lifetimes exist for $\mathrm{NADH}$ and NADPH, and that lifetimes are longer for bound compounds, despite the usual NADPH lifetime increases to higher values (6). This assumption represents the first simplification made in our evaluations, which yields reasonable estimates under the specific conditions when NADPH is nearly constant. Then, eq. $\{1\}$ can be rewritten as:

$$
\begin{aligned}
I(t)=Z+I_{0} \cdot\left\{\left(v_{\mathrm{F}}+\pi_{\mathrm{F}}\right) \cdot \exp \left[-t / \tau_{\mathrm{F}}\right]\right. \\
\left.+\left(v_{\mathrm{F}}+\pi_{\mathrm{F}}\right) \cdot \exp \left[-t / \tau_{\mathrm{B}}\right]\right\}
\end{aligned}
$$

The fraction coefficients $v_{\mathrm{F}}$ and $v_{\mathrm{B}}$ refer to free and bound NADH species, respectively, whereas $\pi_{\mathrm{F}}$ and $\pi_{\mathrm{B}}$ refer to free and bound NADPH molecules, respectively. Assuming that the ratio $\rho=\pi_{\mathrm{B}} / v_{\mathrm{B}}$ can be set proportionally to the concentration ratio of respective bound species (averaged from multiple binding sites on various proteins), $\rho$ can be expressed according to the protocol devised by Duchen's group (6) as (time in ns):

$$
\begin{aligned}
\rho & =\pi_{\mathrm{B}} / \nu_{\mathrm{B}}=[\mathrm{NADPH}]_{\mathrm{B}} /[\mathrm{NADH}]_{\mathrm{B}} \\
& =\left(\tau_{\mathrm{B}}-1.5\right) /\left(4.4-\tau_{\mathrm{B}}\right)
\end{aligned}
$$

Due to the normalization of these coefficients and since by definition $\alpha_{\mathrm{B}}=v_{\mathrm{B}}+\pi_{\mathrm{B}}$, it is also valid to imply:

$$
v_{\mathrm{B}}=\alpha_{\mathrm{B}} /\left(1+\pi_{\mathrm{B}} / v_{\mathrm{B}}\right)=\alpha_{\mathrm{B}} /(1+\rho)
$$

On the basis of the Scatchard equation, assumed for multiple protein binding sites, one can estimate the average free NADH concentration as:

$$
\begin{aligned}
v_{\mathrm{F}} \sim[\mathrm{NADH}]_{\mathrm{F}} & =1 /\left\{\left(K_{\mathrm{a}} \cdot P_{0} \cdot[\mathrm{NADH}]_{\mathrm{B}}{ }^{-1}\right)-K_{\mathrm{a}}\right\} \\
& =1 /\left(K_{\mathrm{a}} \cdot P_{0} \cdot v_{\mathrm{B}}{ }^{-1}-K_{\mathrm{a}}\right)
\end{aligned}
$$

where $K_{\mathrm{a}}$ is the average association constant, and $P_{0}$ is the protein concentration. By substituting eqs. $\{3\}$ and $\{4\}$ into eq. $\{5\}$, we obtain the following expression:

$\left.v_{\mathrm{F}}=1 /\left\{K_{\mathrm{a}} \cdot P_{0} \cdot \alpha_{\mathrm{B}}^{-1} \cdot\left[1+\left(\tau_{\mathrm{B}}-1.5\right) /\left(4.4-\tau_{\mathrm{B}}\right)\right]\right)-K_{\mathrm{a}}\right\}$

The second major simplification in our estimates takes into account the prevailing Complex I contribution so that the parameters $K_{\mathrm{a}}$ and $P_{0}$ correspond to the values obtained in the kinetic model of Markevich and Hoek for Complex I, $0.02 \mu M^{-1}$ and $4541 \mu M$, respectively (45). For this specific case, it is valid to presume that:

$\left.v_{\mathrm{F}}=1 /\left\{90.82 \cdot \alpha_{\mathrm{B}}^{-1} \cdot\left[1+\left(\tau_{\mathrm{B}}-1.5\right) /\left(4.4-\tau_{\mathrm{B}}\right)\right]\right)-0.02\right\}$

In addition, the two-photon excitation fluorescence intensity $F(\mathrm{t})$ can be expressed (26) as:

$$
F(t)=0.5 \cdot[\mathrm{NAD}(\mathrm{P}) \mathrm{H}] \cdot Q \cdot \delta_{2 P} \cdot \zeta \cdot L^{2}(r, t),
$$

where $Q$ is the quantum yield, $\delta_{2 \mathrm{P}}$ is the two-photon excitation fluorophore cros-section, $\zeta$ is the instrumental fluorescence collection efficiency, and $L^{2}(r, t)$ is the average spatiotemporal profile of the excitation laser pulses (6). Consequently, [NAD $(\mathrm{P}) \mathrm{H}]$ can be estimated from eq. $\{8\}$ by substituting $\delta_{2 \mathrm{P}}=0.05$ and $Q=0.02$ (26) to yield:

$$
[\mathrm{NAD}(\mathrm{P}) \mathrm{H}]=2000(t) \cdot \zeta^{-1} \cdot L^{-2}(r, t)
$$

Assuming a very short time interval (shorter than the shortest lifetime) and supposing that the NADH signal can be separated from that of NADPH, we derive free NADH concentration as:

$$
[\mathrm{NADH}]_{\mathrm{F}}=2000 \cdot v_{\mathrm{F}} \cdot I(t=0) \cdot \zeta^{-1} \cdot L^{-2}(r, t=0)
$$

Consequently, one can estimate the change in free NADH concentration, $[\mathrm{NADH}]_{\mathrm{F}}$, under two distinct conditions (1) and (2) to be:

$$
[\mathrm{NADH}(2)]_{\mathrm{F}} /[\mathrm{NADH}(1)]_{\mathrm{F}}=v_{\mathrm{F}}(2) \cdot I(2) / v_{\mathrm{F}}(1) \cdot I(1)
$$


Moreover, the free $\mathrm{NAD}^{+}$concentration, $\left[\mathrm{NAD}^{+}\right]_{\mathrm{F}}$, can be derived from the known intensity of FAD quenching by $\mathrm{NAD}^{+}$. The fluorescence lifetime $\tau^{\mathrm{FAD}}$ for free FAD, which is now the long-lived component (26), has been previously found to decrease in the presence of $\mathrm{NAD}^{+}$due to fluorescence quenching. Assuming Stern-Volmer (i.e., dynamic) quenching, it is valid to postulate that:

$$
\left[\mathrm{NAD}^{+}\right]_{\mathrm{F}}=\left(1 / k_{q}\right) \cdot\left(1 / \tau_{0 \mathrm{~F}}^{\mathrm{FAD}}\right) \cdot\left[\tau_{0 \mathrm{~F}}^{\mathrm{FAD}} / \tau_{\mathrm{F}}^{\mathrm{FAD}}-1\right],
$$

where $k_{\mathrm{q}}$ is the quenching constant, $\tau^{\mathrm{FAD}}{ }_{\mathrm{OF}}$ is the lifetime in the absence of a quencher, and $\tau_{\mathrm{F}}^{\mathrm{FAD}}$ is the long-lived lifetime of FAD emission decay. For FAD, we have assumed that $\tau^{\mathrm{FAD}}{ }_{\mathrm{OF}}$ converges to $2.9 \mathrm{~ns}$. Quenching also affects bound $\mathrm{FAD}$, for which the following relationship can be proposed:

$$
\left[\mathrm{NAD}^{+}\right]_{\mathrm{F}}=\left(1 / k_{q}\right) \cdot\left(1 / \tau^{\mathrm{FAD}}{ }_{0 \mathrm{~B}}\right) \cdot\left[\tau^{\mathrm{FAD}}{ }_{0 \mathrm{~B}} / \tau^{\mathrm{FAD}}{ }_{\mathrm{B}}-1\right],
$$

Bearing in mind that the two simplifications mentioned earlier were made, one can evaluate the substrate pressure $S$ for free compounds as:

$$
\begin{aligned}
S= & {[\mathrm{NADH}]_{\mathrm{F}} /\left[\mathrm{NAD}^{+}\right]_{\mathrm{F}}=4000 \cdot v_{\mathrm{F}} \cdot\left(0.5 \cdot \tau_{\mathrm{FAD}}^{\mathrm{F}}-1\right)^{-1} } \\
& \cdot I(t=0) \cdot k_{q} \cdot \zeta^{-1} \cdot L^{-2}(r, t=0)
\end{aligned}
$$

For relating substrate pressure to two distinct conditions (1) and (2), the constants involved do not need to be known and the following expression can be derived:

$$
\begin{aligned}
\mathrm{S}(2) / \mathrm{S}(1)= & I(2) \cdot v_{\mathrm{F}}(2) \cdot\left(0.5 \cdot \tau(1)_{\mathrm{F}}^{\mathrm{FAD}}-1\right) / \\
& I(1) \cdot v_{\mathrm{F}}(1) \cdot\left(0.5 \cdot \tau(2)_{\mathrm{F}}^{\mathrm{FAD}}-1\right)
\end{aligned}
$$

To estimate the substrate pressure for bound compounds, we use eq. $\{3\}$ and the Scatchard equation for $\mathrm{NAD}^{+}$and obtain:

$$
\begin{aligned}
S_{\mathrm{B}}= & {[\mathrm{NADH}]_{\mathrm{B}} /\left[\mathrm{NAD}^{+}\right]_{\mathrm{B}}=\left\{1 / P_{0}+k_{q} \cdot 2 \cdot\left(\kappa_{\mathrm{a}} \cdot P_{0}\right)^{-1} /\right.} \\
& {\left.\left[0.5 \cdot \tau_{\mathrm{F}}^{\mathrm{FAD}}-1\right]\right\} \cdot[\mathrm{NADPH}]_{\mathrm{B}} \cdot\left(4.4-\tau_{\mathrm{B}}\right) /\left(\tau_{\mathrm{B}}-1.5\right), }
\end{aligned}
$$

where $\kappa_{\mathrm{a}}$ is the association constant for $\mathrm{NAD}^{+}, 0.001 \mu M^{-1}$ (45). Assuming $k_{\mathrm{q}}=\sim 0.01$, the bound substrate pressure can be approximated as:

$$
\begin{aligned}
S_{\mathrm{B}}=\{ & \left.0.00022+0.0044 /\left[0.5 \cdot \tau_{\mathrm{F}}^{\mathrm{FAD}}-1\right]\right\} \\
& \cdot[\mathrm{NADPH}]_{\mathrm{B}} \cdot\left(4.4-\tau_{\mathrm{B}}\right) /\left(\tau_{\mathrm{B}}-1.5\right)
\end{aligned}
$$

We appreciated a certain degree of precision and reproducibility of 2 chFLIM. The previously established method reported the $\mathrm{NADPH}_{\mathrm{B}} / \mathrm{NADH}_{\mathrm{B}}$ ratio for bound species (6). Ultimately, 2chFLIM finally allows a complete set of parameters to be determined. Specifically, we employed the huge "background" of free FAD emission decay given by the longest-lifetime component to estimate nonfluorescent $\mathrm{NAD}^{+}$due to the ability of NAD ${ }^{+}$to quench FAD.

\section{Approximate calibration of 2chFLIM}

We first confirmed the Stern-Volmer dynamic quenching relationship (eq. $\{12\}$ ) with $1 \mathrm{~m} M$ FAD in glycerol by additions of $50 \mu M \mathrm{NAD}^{+}$aliquots, surveying the decreasing lifetime of FAD emission decay, approximated by a single decay component $\tau$, using our Leica TSC SP8 confocal microscopy system with a Becker \& Hickl FLIM attachment (Fig. 7G, H, gray symbols). Then, we employed INS-1E cells permeabilized by using digitonin and either used the natural cytosolic FAD content or added defined FAD amounts reaching 10 or $100 \mu M$ concentrations. Again, we added $\mathrm{NAD}^{+}$aliquots and now we employed 2chFLIM exactly as for the whole cell measurements, that is, including NADH channel as well, while surveying $\tau_{2}{ }^{\mathrm{FAD}}$ decreases (Fig. 7, empty symbols). Measurements were conducted exactly as described earlier for nonpermeabilized cells. The data approximately fit to the Stern-Volmer relationship for dynamic quenching (eq. $\{12\}$ ), to which also fit the $\tau_{2}{ }^{\text {FAD }}$ data (lifetimes for free FAD) from Figure 8F under the assumption that $\mathrm{NAD}^{+}$was around $800 \mu M$. Note also that if this $\mathrm{NAD}^{+}$concentration is overestimated and may be lower in reality, then the corresponding shift of the data would still allow fitting to a slightly different Stern-Volmer relationship (eq. $\{12\}$ ).

\section{Mitochondrial membrane potential $\Delta \Psi_{\mathrm{m}}$ assay}

$\Delta \Psi_{\mathrm{m}}$ was monitored in situ by using tetramethylrhodamine ethyl ester (TMRE) (Thermo Fisher Scientific) in a Fluorolog 322 spectrofluorometer (Horiba Jobin Yvon, Longjumeau, France). Excitation was at $546 \mathrm{~nm}$ (slit width $10 \mathrm{~nm}$ ), and emission was at $574 \mathrm{~nm}$ (slit width $10 \mathrm{~nm}$ ). Cells were incubated with $10 \mathrm{n} M$ TMRE for $15 \mathrm{~min}$. Alternatively, the ratiometric probe JC- 1 indicated a $\Delta \Psi_{\mathrm{m}}$ decrease, which is proportional to the decrease in the ratio of JC-1 fluorescence at 593 versus $537 \mathrm{~nm}(29)$.

\section{High-resolution respirometry}

Cellular $\mathrm{O}_{2}$ consumption was measured by using an Oxygraph-2k (Oroboros, Innsbruck, Austria) after air calibration and background correction (49). Endogenous respiration was recorded first and when required, glucose at the given doses or various agents were added. Nonphosphorylating respiration and nonmitochondrial respiration were determined after the addition of oligomycin and antimycin A (both $1 \mu M$ ), respectively. Nonmitochondrial respiration was subsequently subtracted from all measured rates. An uncoupler, carbonyl cyanide 4-(trifluoromethoxy)phenylhydrazone (FCCP), was titrated at the end of each run to assess the maximum respiratory chain capacity. The assay was conducted either in cell culture medium or in KRH buffer $(135 \mathrm{~m} M \mathrm{NaCl}, 3.6 \mathrm{~m} M \mathrm{KCl}, 10 \mathrm{~m} M$ HEPES, $0.5 \mathrm{~m} M$ $\mathrm{MgCl}_{2}, 1.5 \mathrm{mMCaCl}, 0.5 \mathrm{mM} \mathrm{NaH} \mathrm{PO}_{4}, \mathrm{pH}$ 7.4) containing $0.1 \%$ fatty-acid-free bovine serum albumin (BSA).

Islet respiratory analysis was performed on an Agilent Seahorse XF24 analyzer according to the manufacturer's manual. Fifty islets were seeded into islet capture microplates; analysis was done in KRH media containing $3 \mathrm{mM}$ glucose and 1\% FBS. The final concentration of glucose was $25 \mathrm{~m} M$, whereas the standard testing included sequential 
additions of $5 \mu M$ oligomycin, $1 \mu M$ FCCP, and $5 \mu M$ antimycin plus $5 \mu M$ rotenone.

\section{Quantification of ATP}

An ATP Bioluminescence Assay kit HSII (Roche, Basel, Switzerland) was used to quantify ATP levels. Cells were lysed by boiling in EDTA buffer $(100 \mathrm{~m} M$ Tris-Cl, $4 \mathrm{~m} M$ EDTA, pH 7.75) for 2 min. Bioluminescence was determined by using a Synergy HT luminescence microplate reader (Bio-TEK/Agilent, Winooski, VT).

For the monitoring of ATP in cytosolic or mitochondrial matrix ROI, cells were transfected with cytosolic or mitochondrial ATeam plasmids (a kind gift from Prof. Hiromi Imamura, Kyoto University, Japan) (66), using Lipofectamine 2000. ATeam fluorescence was monitored with a Leica SP5 confocal microscope in lambda scan mode $(X Y \lambda)$. Excitation was set to $405 \mathrm{~nm}$, whereas emission was collected between 453 and $608 \mathrm{~nm}$ ( $5 \mathrm{~nm}$ bandwidth) with a step of $3.88 \mathrm{~nm}$. Integral fluorescence intensity corresponding to the excitation maximum at $\sim 478 \mathrm{~nm}$ (or its ratio to emission maximum at $\sim 522 \mathrm{~nm}$; that is, YFP/CFP emission ratio) is inversely related to the local ATP cytosolic or matrix concentration. The former has also excluded the ATP signal from insulin granules, since emission from their loci is negligible.

\section{Quantification of insulin release}

Cells were seeded at $0.3 \times 10^{6}$ cells/well in poly-L-lysinecoated 12-well plates 2 days before the experiment. Cells were washed with the $\mathrm{KRH}^{\mathrm{BSA}}$ buffer without glucose and then incubated in $\mathrm{KRH}^{\mathrm{BSA}}$ with the desired glucose concentrations. After chosen the time period, insulin levels in the media were determined by using the Rat Insulin High Sensitivity ELISA kit (BioVendor, Brno, Czech Republic) (48).

\section{Quantification of SOD activity}

An SOD assay kit (Cayman, Ann Arbor, MI) was used to estimate SOD activity. $\mathrm{KCN}$ additions were performed to distinguish MnSOD activity from total SOD activity.

\section{Quantification of cellular metabolites and ${ }^{13} \mathrm{C}$-incorporation}

Quantification of pyruvate, OAA, citrate, fumarate, and malate from INS-1E cells was performed by using gas chromatography-mass spectrometry (GC-MS). Internal standard DL-malic acid-2,3,3- $d_{3}(10 \mu \mathrm{L}$ of $300 \mu \mathrm{g} / \mathrm{mL})$ was added. The cell samples were extracted with water/methanol/ chloroform $(1: 1: 2, \mathrm{w} / \mathrm{w} / \mathrm{w})$ and centrifuged $\left(3000 \mathrm{~g}, 5^{\circ} \mathrm{C}\right.$, $10 \mathrm{~min}$ ). The upper polar phase was transferred to glass vials and lyophilized. Analytes were derivatized with pyridine/ $\mathrm{N}, \mathrm{O}-$ bis (trimethylsilyl) acetamide/chlorotrimethylsilane $(3: 2: 1, \mathrm{v} / \mathrm{v} / \mathrm{v})$ at $60^{\circ} \mathrm{C}$ for $70 \mathrm{~min}$.

These samples $(7 \mu \mathrm{L})$ were directly injected in split mode into the gas chromatograph coupled to a mass spectrometer (GC 6890N, MSD 5973N; Agilent Technologies, Santa Clara, CA). The 95\% methyl-, 5\% phenyl-polysiloxane column $(15 \mathrm{~m} \times 250 \mu \mathrm{m} \times 0.25 \mu \mathrm{m})$ was used for the chromatographic separation. The initial temperature of $100^{\circ} \mathrm{C}$ was held for $1 \mathrm{~min}$, then increased by $10^{\circ} \mathrm{C}$ per minute to $180^{\circ} \mathrm{C}$, and finally held for $1 \mathrm{~min}$. Finally, the postcolumn temperature was increased to $300^{\circ} \mathrm{C}$, and this temperature was held for
2 min. The total run time of analysis was 12 min with a flow rate of helium at $1 \mathrm{~mL} / \mathrm{min}$. Ions were generated by using the electron-ionization mode at $70 \mathrm{eV}$ with the ion source maintained at $230^{\circ} \mathrm{C}$. Ions were measured by using SIM acquisition mode. Specific ions have been selected in verified mass spectra of metabolites: pyruvate [131], OAA [217], citrate [273], fumarate [245], 2OG [347], malate [335], and DL-malic acid-2,3,3- $d_{3}$ [338]. Retention times of selected metabolites were confirmed with commercial standards, and detectable specific mass ions were chosen according to their mass spectra.

\section{Quantification of ${ }^{13} \mathrm{C}$-incorporation}

The reductive carboxylation by IDH2, a counter-Krebs cycle direction reaction consuming 2OG, was semiquantified as the percentage of surplus of accumulated ${ }^{13} \mathrm{C}$-citrate and ${ }^{13} \mathrm{C}$-malate from $1-{ }^{13} \mathrm{C}$-glutamine after $6 \mathrm{~h}$ (59). Internal standard malate- $d 3$ was added and samples were treated and measured as described earlier. The ratios between data obtained in 20 versus $3 \mathrm{mM}$ glucose were calculated after subtraction of the natural ${ }^{13} \mathrm{C}$ content from the rough ${ }^{13} \mathrm{C}$ amounts related as the percentage of ${ }^{12} \mathrm{C}+{ }^{13} \mathrm{C}$ amount for each sample; and then the respective ratios were subsequently calculated. Thus, for malate, 2OG, and citrate $(\mathrm{C} 4$, C5, and C6 compounds) the derivatized fragmented ions 335 , 348 , and 273 (alternatively 465) contain altogether 12, 13, and 11 (alternatively 17) carbons, respectively. Hence, their natural ${ }^{13} \mathrm{C}$ content is $13.2 \%, 14.3 \%$, and $12.1 \%$ (alternatively $18.7 \%$ ), respectively. Thus, for incorporation into malate, ${ }^{13} \mathrm{C}$-labeled malate ions 336 were traced versus ions 335; 349 versus ions 348 were traced for 2OG; as well as citrate originating ions 274 (alternatively 466) were compared with ${ }^{12} \mathrm{C}$ citrate ions 273 [465].

\section{Statistical analysis}

Analysis of variance was carried out with subsequent pairwise multiple comparisons (Tukey's or Holm-Sidak's test) by using SigmaStat 3.1 (Systat Software, San Jose, CA). Error bars in figures represent standard deviations.

\section{Authors' Contributions}

L.P.-H., H.E., A.D., J.T., J.J., and T.Š. performed MitoSOX Red confocal assays; H.E. performed mito-HyPer, iNAP1, iNAP3, and 2chFLIM assays; B.H., H.E., L.P.-H., V.K., and L.P. performed the MitoP/MitoB experiments, whereas B.H., V.K., and L.P. undertook other LC-MS assays; A.D., J.T., J.J., and T.Š. performed respiration experiments; A.D. worked with ATeam; B.H., H.E., and J.T. carried out cell silencing; L.P.-H., H.E., and K.S. performed auxiliary experiments; K.S. designed Figure 1; and K.S., K.G., and J.Š. performed metabolomics and ${ }^{13} \mathrm{C}$-incorporation studies. L.P.-H., B.H., H.E., K.S., and P.J. designed experiments; P.J. developed the theory of 2 chFLIM assessment of $\mathrm{NAD}^{+}$and other parameters, calculated data, drew figures, and wrote the article.

\section{Acknowledgments}

The authors gratefully acknowledge Prof. Dr. Libor Vítek and Dr. Aleš Dvořák, 1st Faculty of Medicine, Charles University, Prague, for enabling them to use GC-MS; Prof. Yi 
Yang, Synthetic Biology and Biotechnology Laboratory, State Key Laboratory of Bioreactor Engineering, Shanghai Collaborative Innovation Center for Biomanufacturing Technology, East China University of Science and Technology, Shanghai, China, for providing plasmids for iNAP fluorescent probes; Prof. Hiromi Imamura, Department of Functional Biology, Graduate School of Biostudies, Kyoto University, Kyoto, Japan, for providing ATeam plasmids; Dr. Enyedi, Semmelweis University, Hungary, for providing mito-HyPer and mito-SypHer plasmids; and finally, the excellent technical assistance of Lenka Josková and Jana Vaicová.

\section{Author Disclosure Statement}

No competing financial interests exist.

\section{Funding Information}

This project was supported by grants from the Grant Agency of the Czech No. 16-06700S to L.P.-H., and 1701813S plus 20-00408S to P.J. and by the institutional support project RVO67985823.

\section{Supplementary Material}

Supplementary Data

Supplementary Figure S1

Supplementary Figure S2

Supplementary Figure S3

Supplementary Figure S4

Supplementary Figure S5

Supplementary Figure S6

Supplementary Figure S7

Supplementary Figure S8

Supplementary Table S1

\section{References}

1. Aon MA, Stanley BA, Sivakumaran V, Kembro JM, O'Rourke B, Paolocci N, and Cortassa S. Glutathione/ thioredoxin systems modulate mitochondrial $\mathrm{H} 2 \mathrm{O} 2$ emission: an experimental-computational study. J Gen Physiol 139: 479-491, 2012.

2. Ashcroft Frances M and Rorsman P. Diabetes mellitus and the $\beta$ cell: the last ten years. Cell 148: 1160-1171, 2012.

3. Belousov VV, Fradkov AF, Lukyanov KA, Staroverov DB, Shakhbazov KS, Terskikh AV, and Lukyanov S. Genetically encoded fluorescent indicator for intracellular hydrogen peroxide. Nat Methods 3: 281-286, 2006.

4. Bilan DS and Belousov VV. HyPer family probes: state of the art. Antioxid Redox Signal 24: 731-751, 2016.

5. Bindokas VP, Kuznetsov A, Sreenan S, Polonsky KS, Roe MW, and Philipson LH. Visualizing superoxide production in normal and diabetic rat islets of langerhans. $\mathrm{J} \mathrm{Biol} \mathrm{Chem}$ 278: 9796-9801, 2003.

6. Blacker TS, Mann ZF, Gale JE, Ziegler M, Bain AJ, Szabadkai G, and Duchen MR. Separating NADH and NADPH fluorescence in live cells and tissues using FLIM. Nat Commun 5: 3936, 2014.

7. Bleier L and Dröse S. Superoxide generation by complex III: from mechanistic rationales to functional consequences. Biochim Biophys Acta Bioenerg 1827: 1320-1331, 2013.
8. Brand MD. Mitochondrial generation of superoxide and hydrogen peroxide as the source of mitochondrial redox signaling. Free Radic Biol Med 100: 14-31, 2016.

9. Brand MD, Goncalves RLS, Orr AL, Vargas L, Gerencser AA, Jensen MB, Wang YT, Melov S, Turk CN, Matzen JT, Dardov VJ, Petrassi HM, Meeusen SL, Perevoshchikova IV, Jasper H, Brookes PS, and Ainscow EK. Suppressors of superoxide- $\mathrm{H}_{2} \mathrm{O}_{2}$ production at site I Q of mitochondrial complex I protect against stem cell hyperplasia and ischemia-reperfusion injury. Cell Metab 24: 582-592, 2016.

10. Brun $\mathrm{T}$ and Maechler P. Beta-cell mitochondrial carriers and the diabetogenic stress response. Biochim Biophys Acta 1863: 2540-2549, 2016.

11. Cardoso AR, Chausse B, da Cunha FM, Luévano-Martínez LA, Marazzi TBM, Pessoa PS, Queliconi BB, and Kowaltowski AJ. Mitochondrial compartmentalization of redox processes. Free Radic Biol Med 52: 2201-2208, 2012.

12. Chen WW, Freinkman E, Wang T, Birsoy K, and Sabatini DM. Absolute quantification of matrix metabolites reveals the dynamics of mitochondrial metabolism. Cell 166: 1324-1337.e11, 2016.

13. Chouchani ET, Pell VR, Gaude E, Aksentijević D, Sundier SY, Robb EL, Logan A, Nadtochiy SM, Ord ENJ, Smith AC, Eyassu F, Shirley R, Hu CH, Dare AJ, James AM, Rogatti S, Hartley RC, Eaton S, Costa ASH, Brookes PS, Davidson SM, Duchen MR, Saeb-Parsy K, Shattock MJ, Robinson AJ, Work LM, Frezza C, Krieg T, and Murphy MP. Ischaemic accumulation of succinate controls reperfusion injury through mitochondrial ROS. Nature 515: 431-435, 2014.

14. Cochemé HM, Logan A, Prime TA, Abakumova I, Quin C, McQuaker SJ, Patel JV, Fearnley IM, James AM, Porteous CM, Smith RAJ, Hartley RC, Partridge L, and Murphy MP. Using the mitochondria-targeted ratiometric mass spectrometry probe MitoB to measure $\mathrm{H} 2 \mathrm{O} 2$ in living Drosophila. Nat Protoc 7: 946-958, 2012.

15. Deglasse J-P, Roma LP, Pastor-Flores D, Gilon P, Dick TP, and Jonas J-C. Glucose acutely reduces cytosolic and mitochondrial $\mathrm{H} 2 \mathrm{O} 2$ in rat pancreatic beta cells. Antioxid Redox Signal 30: 297-313, 2019.

16. Dey S, Sidor A, and O'Rourke B. Compartment-specific control of reactive oxygen species scavenging by antioxidant pathway enzymes. J Biol Chem 291: 11185-11197, 2016.

17. Dlasková A, Engstová H, Špaček T, Kahancová A, Pavluch V, Smolková K, Špačková J, Bartoš M, Hlavatá LP, and Ježek P. 3D super-resolution microscopy reflects mitochondrial cristae alternations and mtDNA nucleoid size and distribution. Biochim Biophys Acta Bioenerg 1859: 829844, 2018.

18. Dlasková A, Hlavatá L, and Ježek P. Oxidative stress caused by blocking of mitochondrial Complex I H+ pumping as a link in aging/disease vicious cycle. Int $J$ Biochem Cell Biol 40: 1792-1805, 2008.

19. Dlasková A, Špaček T, Šantorová J, Plecitá-Hlavatá L, Berková Z, Saudek F, Lessard M, Bewersdorf J, and Ježek P. 4Pi microscopy reveals an impaired three-dimensional mitochondrial network of pancreatic islet $\beta$-cells, an experimental model of type-2 diabetes. Biochim Biophys Acta Bioenerg 1797: 1327-1341, 2010.

20. Doyle ME and Egan JM. Mechanisms of action of glucagon-like peptide 1 in the pancreas. Pharmacol Ther 113: 546-593, 2007. 
21. Dröse $S$ and Brandt U. Molecular mechanisms of superoxide production by the mitochondrial respiratory chain. In: $M i$ tochondrial Oxidative Phosphorylation. Advances in Experimental Medicine and Biology vol. 748, edited by Kadenbach B. New York, NY: Springer, 2012, pp. 145-169.

22. Ferdaoussi M, Dai X, Jensen MV, Wang R, Peterson BS, Huang C, Ilkayeva O, Smith $\mathrm{N}$, Miller N, Hajmrle C, Spigelman AF, Wright RC, Plummer G, Suzuki K, Mackay JP, van de Bunt M, Gloyn AL, Ryan TE, Norquay LD, Brosnan MJ, Trimmer JK, Rolph TP, Kibbey RG, Manning Fox JE, Colmers WF, Shirihai OS, Neufer PD, Yeh ETH, Newgard $\mathrm{CB}$, and MacDonald PE. Isocitrate-to-SENP1 signaling amplifies insulin secretion and rescues dysfunctional $\beta$ cells. J Clin Invest 125: 3847-3860, 2015.

23. Fridlyand LE and Philipson LH. Does the glucosedependent insulin secretion mechanism itself cause oxidative stress in pancreatic beta-cells? Diabetes 53: 1942-1948, 2004.

24. Gerencser AA. Metabolic activation-driven mitochondrial hyperpolarization predicts insulin secretion in human pancreatic beta-cells. Biochim Biophys Acta Bioenerg 1859: 817-828, 2018.

25. Guillaud F, Dröse S, Kowald A, Brandt U, and Klipp E. Superoxide production by cytochrome bc1 complex: a mathematical model. Biochim Biophys Acta Bioenerg 1837: 1643-1652, 2014.

26. Huang S, Heikal AA, and Webb WW. Two-photon fluorescence spectroscopy and microscopy of $\mathrm{NAD}(\mathrm{P}) \mathrm{H}$ and flavoprotein. Biophys J 82: 2811-2825, 2002.

27. Ivarsson R, Quintens R, Dejonghe $S$, Tsukamoto $K$, in ' $t$ Veld P, Renström E, and Schuit FC. Redox control of exocytosis: regulatory role of NADPH, thioredoxin, and glutaredoxin. Diabetes 54: 2132-2142, 2005.

28. Ježek J, Dlasková A, Zelenka J, Jabůrek M, and Ježek P. $\mathrm{H} 2 \mathrm{O} 2$-activated mitochondrial phospholipase iPLA $2 \gamma$ prevents lipotoxic oxidative stress in synergy with UCP2, amplifies signaling via G-protein-coupled receptor GPR40, and regulates insulin secretion in pancreatic $\beta$-cells. Antioxid Redox Signal 23: 958-972, 2015.

29. Ježek J, Engstová H, and Ježek P. Antioxidant mechanism of mitochondria-targeted plastoquinone SkQ1 is suppressed in aglycemic HepG2 cells dependent on oxidative phosphorylation. Biochim Biophys Acta Bioenerg 1858: 750-762, 2017.

30. Ježek P, Dlasková A, and Plecitá-Hlavatá L. Redox homeostasis in pancreatic $\beta$ cells. Oxid Med Cell Longev 2012: 932838, 2012.

31. Ježek P and Hlavatá L. Mitochondria in homeostasis of reactive oxygen species in cell, tissues, and organism. Int $J$ Biochem Cell Biol 37: 2478-2503, 2005.

32. Jezek P, Jaburek M, Holendova B, and Plecita-Hlavata L. Fatty acid-stimulated insulin secretion vs. lipotoxicity. Molecules 23: 1483, 2018.

33. Ježek $\mathrm{P}$, Jabůrek $\mathrm{M}$, and Plecitá-Hlavatá L. Contribution of oxidative stress and impaired biogenesis of pancreatic $\beta$-cells to type 2 diabetes. Antioxid Redox Signal 31: 722 $751,2019$.

34. Jitrapakdee S, Wutthisathapornchai A, Wallace JC, and MacDonald MJ. Regulation of insulin secretion: role of mitochondrial signalling. Diabetologia 53: 1019-1032, 2010.

35. Joseph JW, Jensen MV, Ilkayeva O, Palmieri F, Alárcon C, Rhodes CJ, and Newgard CB. The mitochondrial citrate/ isocitrate carrier plays a regulatory role in glucosestimulated insulin secretion. J Biol Chem 281: 3562435632, 2006.
36. Kalyanaraman B, Dranka BP, Hardy M, Michalski R, and Zielonka J. HPLC-based monitoring of products formed from hydroethidine-based fluorogenic probes - the ultimate approach for intra- and extracellular superoxide detection. Biochimt Biophys Acta 1840: 739-744, 2014.

37. Koshkin V, Wang X, Scherer PE, Chan CB, and Wheeler MB. Mitochondrial functional state in clonal pancreatic $\beta$-cells exposed to free fatty acids. J Biol Chem 278: 19709-19715, 2003.

38. Kostyuk AI, Panova AS, Bilan DS, and Belousov VV. Redox biosensors in a context of multiparameter imaging. Free Radic Biol Med 128: 23-39, 2018.

39. Kozlov AV, Lancaster JR, Meszaros AT, and Weidinger A. Mitochondria-meditated pathways of organ failure upon inflammation. Redox Biol 13: 170-181, 2017.

40. Kussmaul L and Hirst J. The mechanism of superoxide production by NADH:ubiquinone oxidoreductase (complex I) from bovine heart mitochondria. Proc Natl Acad Sci U S A 103: 7607-7612, 2006.

41. Lombard DB and Zwaans BMM. SIRT3: as simple as it seems? Gerontology 60: 56-64, 2014.

42. MacDonald MJ. Feasibility of a mitochondrial pyruvate malate shuttle in pancreatic islets. Further implication of cytosolic NADPH in insulin secretion. J Biol Chem 270: 20051-20058, 1995.

43. Macdonald MJ, Hasan NM, and Longacre MJ. Studies with leucine, beta-hydroxybutyrate and ATP citrate lyasedeficient beta cells support the acetoacetate pathway of insulin secretion. Biochim Biophys Acta 1780: 966-972, 2008.

44. Maechler P. Glutamate pathways of the beta-cell and the control of insulin secretion. Diabetes Res Clin Pract 131: 149-153, 2017.

45. Markevich NI and Hoek JB. Computational modeling analysis of mitochondrial superoxide production under varying substrate conditions and upon inhibition of different segments of the electron transport chain. Biochim Biophys Acta 1847: 656-679, 2015.

46. Newman JC and Verdin E. $\beta$-Hydroxybutyrate: a signaling metabolite. Anпu Rev Nutr 37: 51-76, 2017.

47. Patterson GH, Knobel SM, Arkhammar P, Thastrup O, and Piston DW. Separation of the glucose-stimulated cytoplasmic and mitochondrial $\mathrm{NAD}(\mathrm{P}) \mathrm{H}$ responses in pancreatic islet beta cells. Proc Natl Acad Sci U S A 97: 5203-5207, 2000.

48. Plecita-Hlavata L, Jaburek M, Holendova B, Tauber J, Pavluch V, Berkova Z, Cahova M, Schroeder K, Brandes RP, Siemen D, and Jezek P. Glucose-stimulated insulin secretion fundamentally requires $\mathrm{H}_{2} \mathrm{O}_{2}$ signaling by NADPH oxidase 4. Diabetes 2020 [Epub ahead of print]; DOI: $10.2337 / \mathrm{db} 19-1130$.

49. Plecitá-Hlavatá L, Ježek J, and Ježek P. Aglycemia keeps mitochondrial oxidative phosphorylation under hypoxic conditions in HepG2 cells. J Bioenerg Biomembr 47: 467476, 2015.

50. Plecitá-Hlavatá L and Ježek P. Integration of superoxide formation and cristae morphology for mitochondrial redox signaling. Int J Biochem Cell Biol 80: 31-50, 2016.

51. Plecitá-Hlavatá L, Lessard M, Santorová J, Bewersdorf J, and Jezek P. Mitochondrial oxidative phosphorylation and energetic status are reflected by morphology of mitochondrial network in INS-1E and HEP-G2 cells viewed by 4Pi microscopy. Biochim Biophys Acta 1777: 834-846, 2008. 
52. Prentki M, Matschinsky Franz M, and Madiraju SRM. Metabolic signaling in fuel-induced insulin secretion. Cell Metab 18: 162-185, 2013.

53. Rocheleau JV, Head WS, and Piston DW. Quantitative $\mathrm{NAD}(\mathrm{P}) \mathrm{H} /$ flavoprotein autofluorescence imaging reveals metabolic mechanisms of pancreatic islet pyruvate response. J Biol Chem 279: 31780-31787, 2004.

54. Roma Leticia P, Duprez J, Takahashi Hilton K, Gilon P, Wiederkehr A, and Jonas J-C. Dynamic measurements of mitochondrial hydrogen peroxide concentration and glutathione redox state in rat pancreatic $\beta$-cells using ratiometric fluorescent proteins: confounding effects of $\mathrm{pH}$ with HyPer but not roGFP1. Biochem J 441: 971-978, 2012.

55. Rutter Guy A, Pullen Timothy J, Hodson David J, and Martinez-Sanchez A. Pancreatic $\beta$-cell identity, glucose sensing and the control of insulin secretion. Biochem J 466: 203-218, 2015.

56. Sakai K, Matsumoto K, Nishikawa T, Suefuji M, Nakamaru K, Hirashima Y, Kawashima J, Shirotani T, Ichinose K, Brownlee M, and Araki E. Mitochondrial reactive oxygen species reduce insulin secretion by pancreatic beta-cells. Biochem Biophys Res Commun 300: 216-222, 2003.

57. Sanderson TH, Reynolds CA, Kumar R, Przyklenk K, and Hüttemann M. Molecular mechanisms of ischemiareperfusion injury in brain: pivotal role of the mitochondrial membrane potential in reactive oxygen species generation. Mol Neurobiol 47: 9-23, 2013.

58. Santos LRB, Muller C, de Souza AH, Takahashi HK, Spégel P, Sweet IR, Chae H, Mulder H, and Jonas J-C. NNT reverse mode of operation mediates glucose control of mitochondrial NADPH and glutathione redox state in mouse pancreatic $\beta$-cells. Mol Metab 6: 535-547, 2017.

59. Smolkova K, Dvorak A, Zelenka J, Vitek L, and Jezek P. Reductive carboxylation and 2-hydroxyglutarate formation by wild-type IDH2 in breast carcinoma cells. Int J Biochem Cell Biol 65: 125-133, 2015.

60. Špaček T, Šantorová J, Zacharovová K, Berková Z, Hlavatá L, Saudek F, and Ježek P. Glucose-stimulated insulin secretion of insulinoma INS-1E cells is associated with elevation of both respiration and mitochondrial membrane potential. Int J Biochem Cell Biol 40: 1522-1535, 2008.

61. Spégel P, Andersson LE, Storm P, Sharoyko V, Göhring I, Rosengren $\mathrm{AH}$, and Mulder $\mathrm{H}$. Unique and shared metabolic regulation in clonal $\beta$-cells and primary islets derived from rat revealed by metabolomics analysis. Endocrinology 156: 1995-2005, 2015.

62. Spégel $\mathrm{P}$ and Mulder H. Metabolomics analysis of nutrient metabolism in $\beta$-cells. $J$ Mol Biol 432: 1429-1445, 2020.

63. Tao R, Coleman MC, Pennington JD, Ozden O, Park S-H, Jiang H, Kim H-S, Flynn CR, Hill S, Hayes McDonald W, Olivier AK, Spitz DR, and Gius D. Sirt3-mediated deacetylation of evolutionarily conserved lysine 122 regulates MnSOD activity in response to stress. Mol Cell 40: 893904, 2010.

64. Tao R, Zhao Y, Chu H, Wang A, Zhu J, Chen X, Zou Y, Shi M, Liu R, Su N, Du J, Zhou H-M, Zhu L, Qian X, Liu $\mathrm{H}$, Loscalzo J, and Yang Y. Genetically encoded fluorescent sensors reveal dynamic regulation of NADPH metabolism. Nat Methods 14: 720-728, 2017.

65. Treberg JR, Quinlan CL, and Brand MD. Evidence for two sites of superoxide production by mitochondrial NADHubiquinone oxidoreductase (complex I). J Biol Chem 286: 27103-27110, 2011.
66. Yoshida $\mathrm{T}$, Alfaqaan $\mathrm{S}$, Sasaoka $\mathrm{N}$, and Imamura $\mathrm{H}$ Application of FRET-based biosensor "ATeam" for visualization of ATP levels in the mitochondrial matrix of living mammalian cells. Methods Mol Biol 1567: 231-243, 2017.

67. Zielonka J, Joseph J, Sikora A, Hardy M, Ouari O, Vasquez-Vivar J, Cheng G, Lopez M, and Kalyanaraman B. Mitochondria-targeted triphenylphosphonium-based compounds: syntheses, mechanisms of action, and therapeutic and diagnostic applications. Chem Rev 117: 10043-10120, 2017.

68. Zielonka J and Kalyanaraman B. Hydroethidine- and MitoSOX-derived red fluorescence is not a reliable indicator of intracellular superoxide formation: another inconvenient truth. Free Radic Biol Med 48: 983-1001, 2010.

Address correspondence to: Dr. Petr Ježek

Department of Mitochondrial Physiology, No. 75 Institute of Physiology of the Czech Academy of Sciences Vídeňská 1083

Prague 414220

Czech Republic

E-mail: jezek@biomed.cas.cz

Date of first submission to ARS Central, May 3, 2019; date of final revised submission, June 3, 2020; date of acceptance, June 8, 2020.

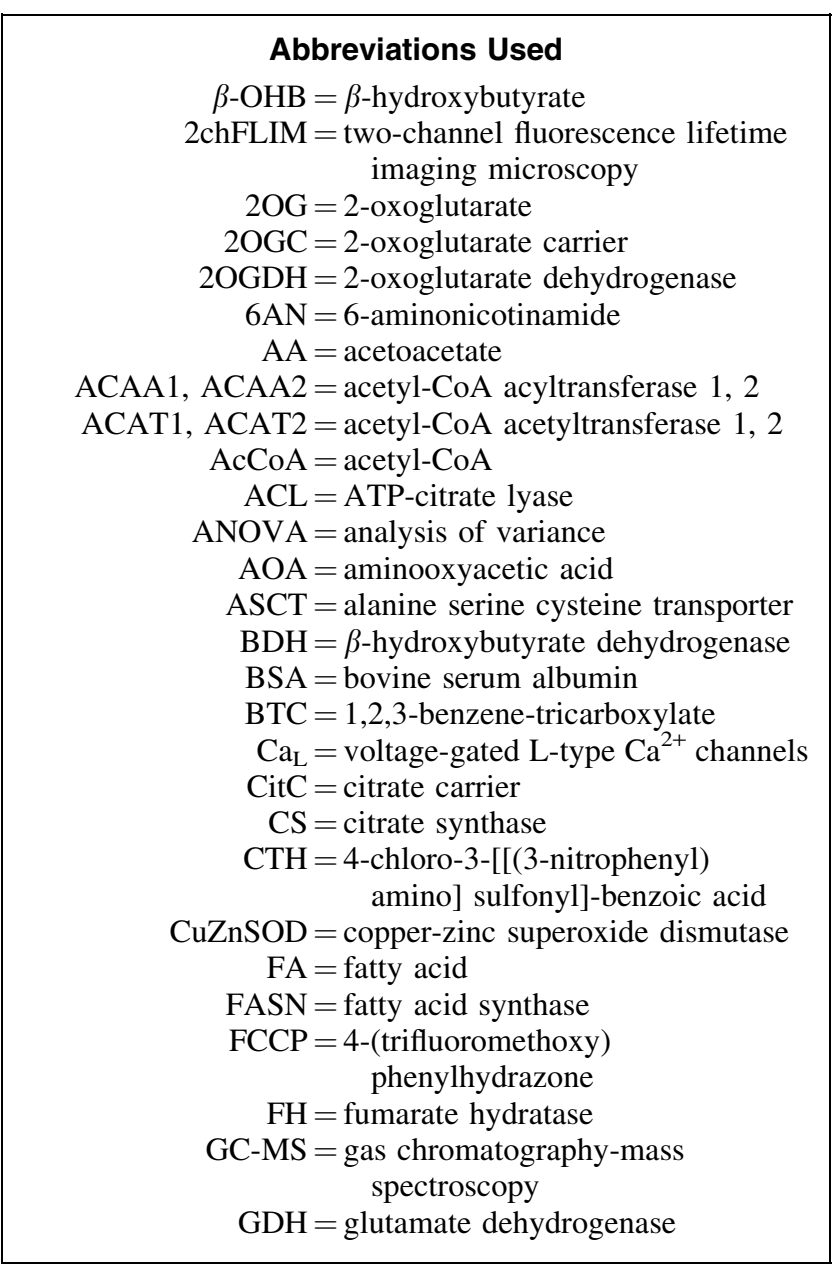




\begin{tabular}{|c|}
\hline $\begin{aligned} \text { Abbreviations Used (Cont.) } \\
\text { Gln C }=\text { glutamine carrier } \\
\text { GLUT }=\text { glucose transporter } \\
\text { GSH }=\text { reduced glutathione } \\
\text { GSIS }=\text { glucose-stimulated insulin secretion } \\
\text { HMG-CoA }=\text { hydroxymethyl-glutaryl-CoA } \\
\text { HMG-CoA L }=\text { hydroxymethyl-glutaryl-CoA lyase } \\
\text { IDH1 }=\text { isocitrate dehydrogenase } 1 \\
\text { IDH2 }=\text { isocitrate dehydrogenase } 2 \\
\text { IDH3 }=\text { isocitrate dehydrogenase } 3 \\
\text { IMM }=\text { inner mitochondrial membrane } \\
\mathrm{K}_{\mathrm{ATP}}=\text { ATP-sensitive potassium } \\
\mathrm{KRH}=\text { Krebs-Ringer HEPES buffer } \\
\mathrm{MAC}=\text { malate aspartate carrier } \\
\mathrm{MDH}=\text { malate dehydrogenase } \\
\mathrm{ME} 1=\text { cytosolic malic enzyme } \\
\mathrm{ME} 3=\text { mitochondrial malic enzyme } \\
\text { NADP }{ }^{+} \text {dependent } \\
\mathrm{MnSOD}=\text { manganese superoxide dismutase } \\
\mathrm{mtDNA}=\text { mitochondrial DNA } \\
\mathrm{NNT}=\text { nicotinamide nucleotide } \\
\text { transhydrogenase } \\
\mathrm{NOX} 4=\text { NADPH oxidase } 4 \\
\mathrm{LC}-\mathrm{MS}=\text { liquid chromatography-mass } \\
\text { spectrometry }\end{aligned}$ \\
\hline
\end{tabular}

\begin{tabular}{|c|}
\hline 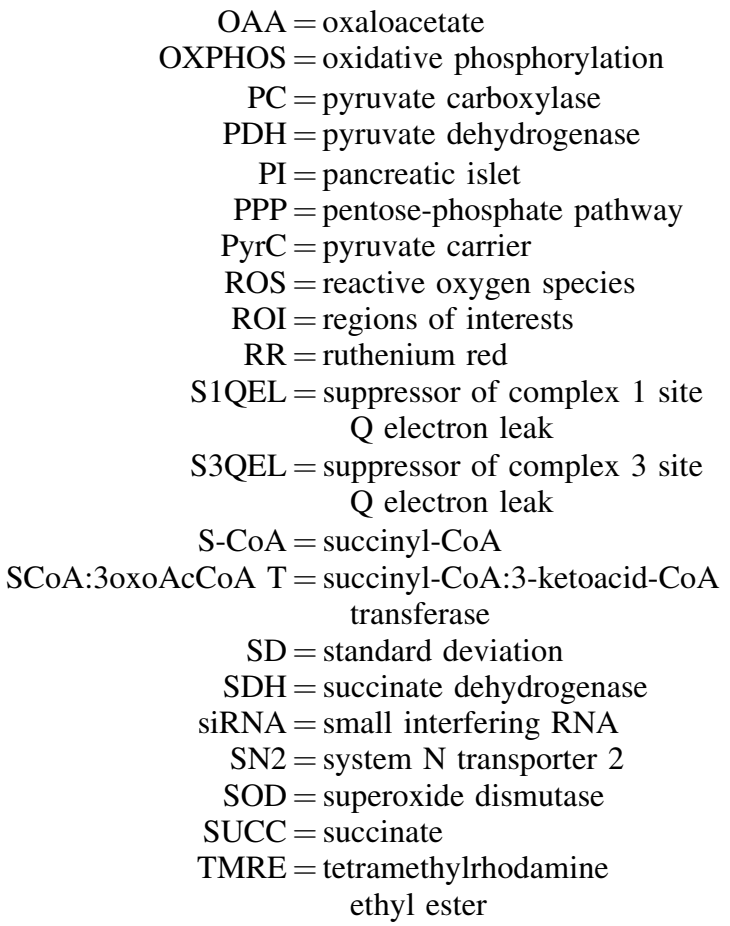 \\
\hline
\end{tabular}

